\title{
Dimethyl Sulfoxide and N-Iodosuccinimide Promoted 5-exo-dig Oxidative Cyclization of Yne-Tethered-Ynamide: Access to Pyrrolidones and Spiro-Pyrrolidones
}

B. Prabagar, Sanatan Nayak, Rangu Prasad and Akhila K. Sahoo*

School of Chemistry, University of Hyderabad, Hyderabad-500046, INDIA

akhilchemistry12@gmail.com

\section{SUPPORTING INFORMATION}




\section{BRUKER MAXIS HRMS REPORT \\ School of Chemistry \\ University of Hyderabad}

Analysis Info

Analysis Name Method

Sample Name Comment
Acquisition Date

D:IDatal2015IDR.AK.SAHOOIJULYPB-6-23.d tune low.m

AKO-1-CHCL3-ACN
Operator

Instrument
7/30/2015 2:58:35 PM

Ramu Sridhar maXis

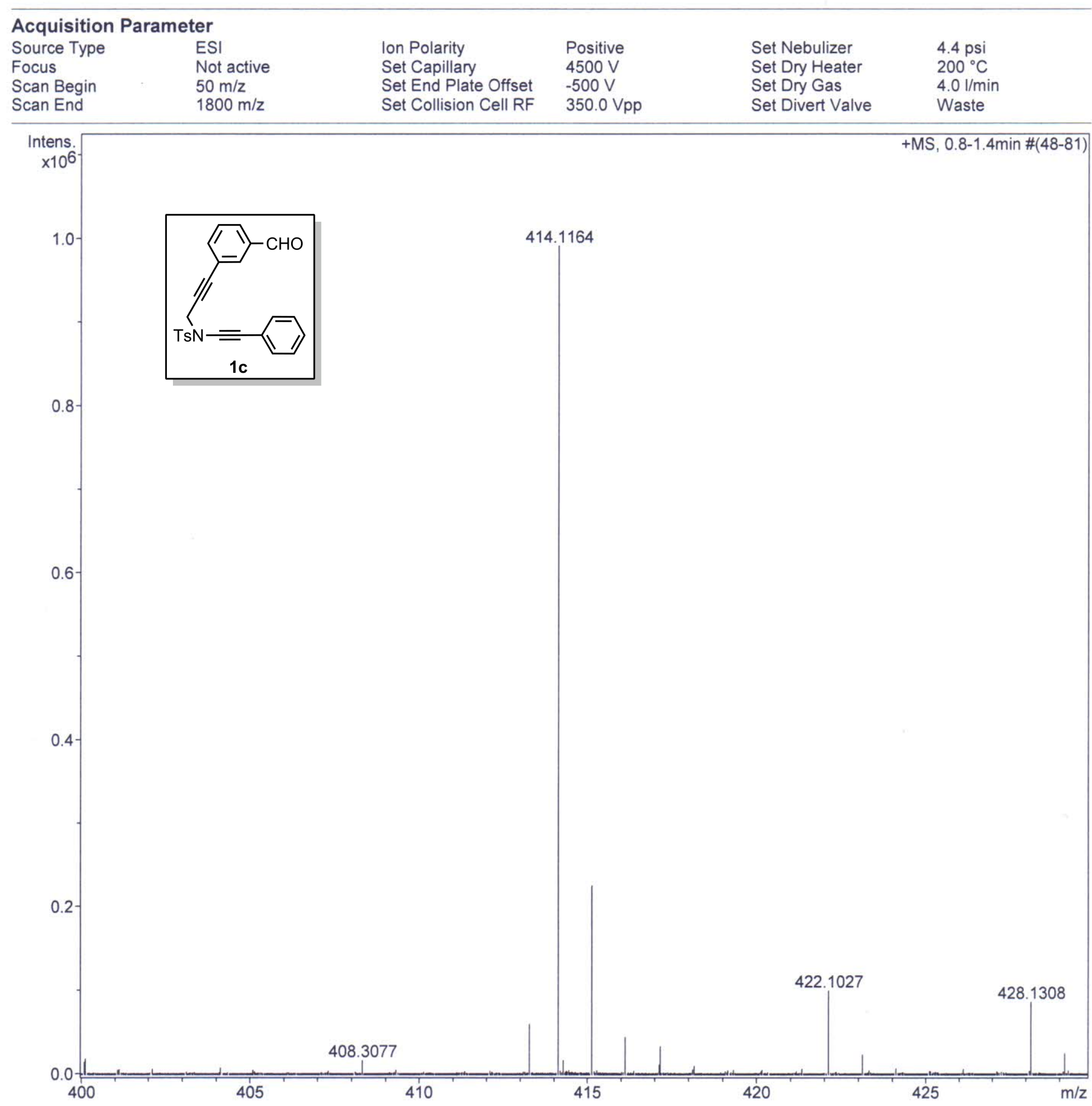




\section{BRUKER MAXIS HRMS REPORT \\ School of Chemistry \\ University of Hyderabad}

Analysis Info

Analysis Name Method

Sample Name

Comment
Acquisition Date

2/20/2015 1:52:01 PM

D:IDataI2015IDR.AK.SAHOOIFEBIPB-6-7R1.d tune_low_Pos.m

Operator

Instrument

Ramu Sridhar

maXis

10138

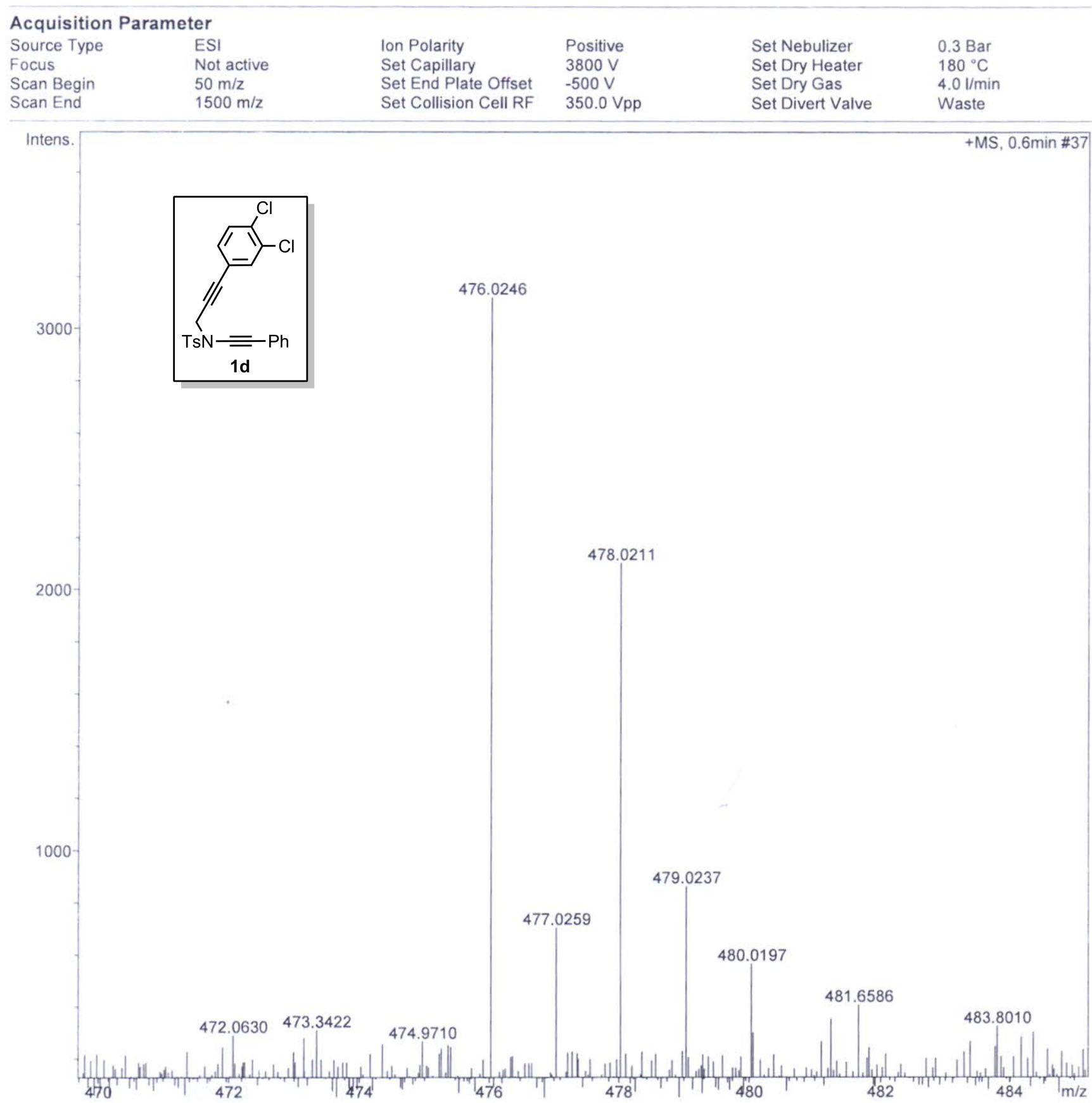




\section{BRUKER MAXIS HRMS REPORT \\ School of Chemistry \\ University of Hyderabad}

Analysis Info

Analysis Name

Method

Sample Name

Comment
Acquisition Date

D:IDatal2015IDR.AK.SAHOOIOctIRP-3-129.d

tune_low.m

RP-3-129-MEOH
Operator

Instrument
10/30/2015 12:30:32 PM

Ramu Sridhar

maXis

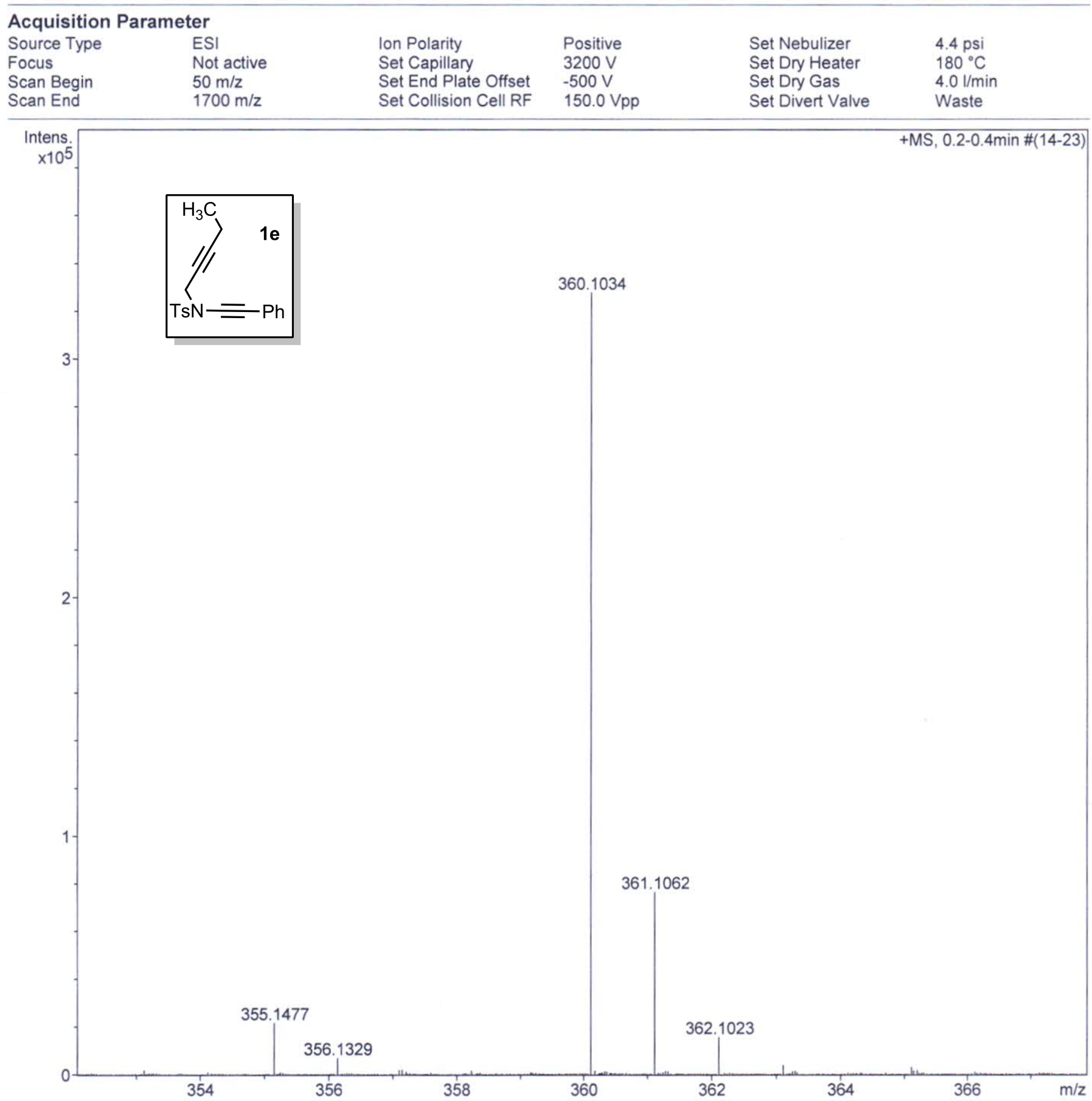




\section{BRUKER MAXIS HRMS REPORT \\ School of Chemistry \\ University of Hyderabad}

\section{Analysis Info}

Analysis Name Method

Sample Name Comment
Acquisition Date 11/5/2015 2:50:22 PM

D:IDatal2015IDR.AK.SAHOOINOVRP-3-140.d tune_low_Pos.m

RP-3-140

Operator

Instrument

Ramu Sridhar

maXis 10138

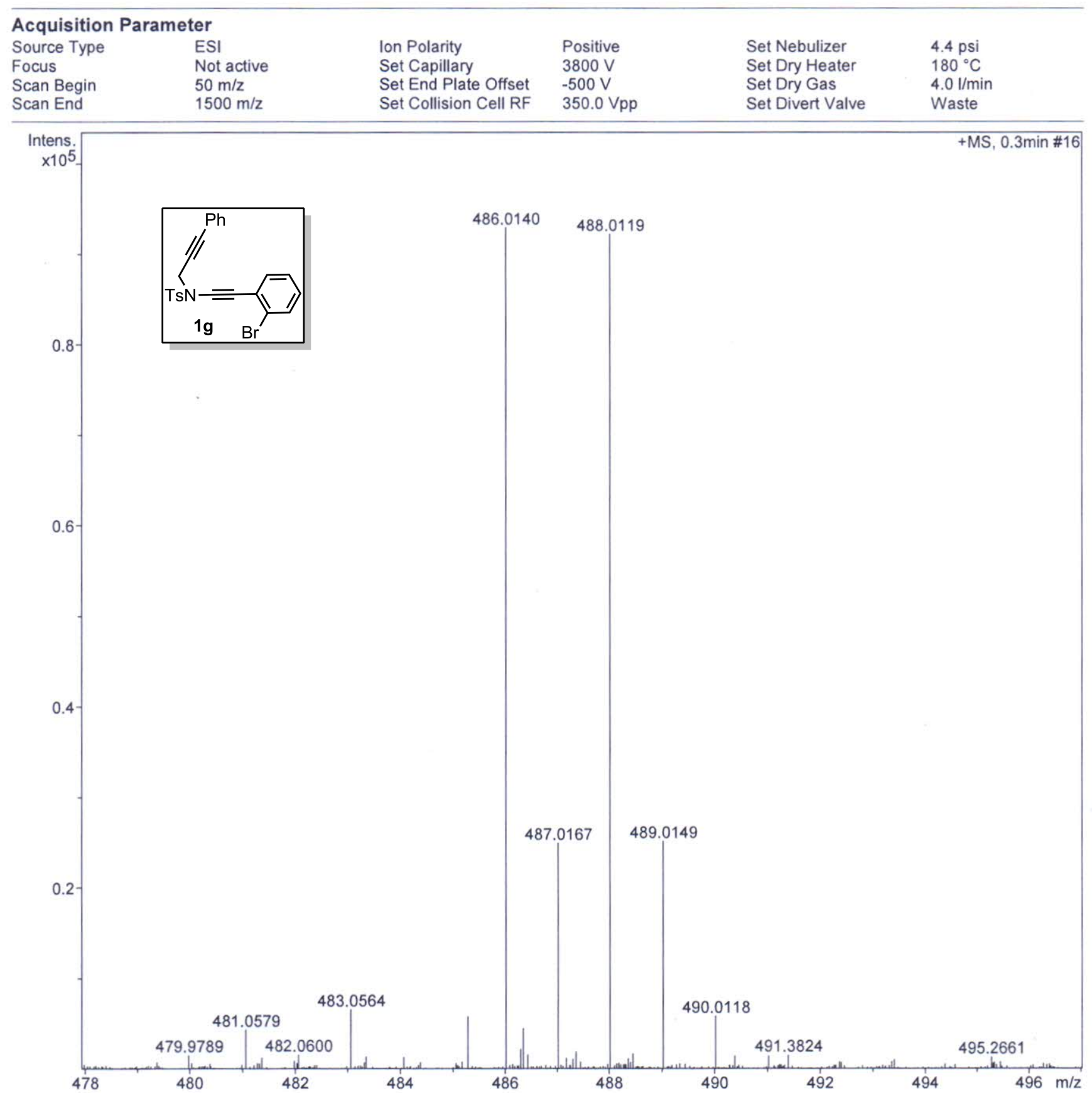




\section{BRUKER MAXIS HRMS REPORT \\ School of Chemistry \\ University of Hyderabad}

\section{Analysis Info}

Analysis Name Method

Sample Name

Comment
Acquisition Date

D:IDatal2015IDR.AK.SAHOOIDECIRP-3-163.d

tune_low.m

RP-3-163-MEOH

Operator

Instrument

Ramu Sridhar

maXis

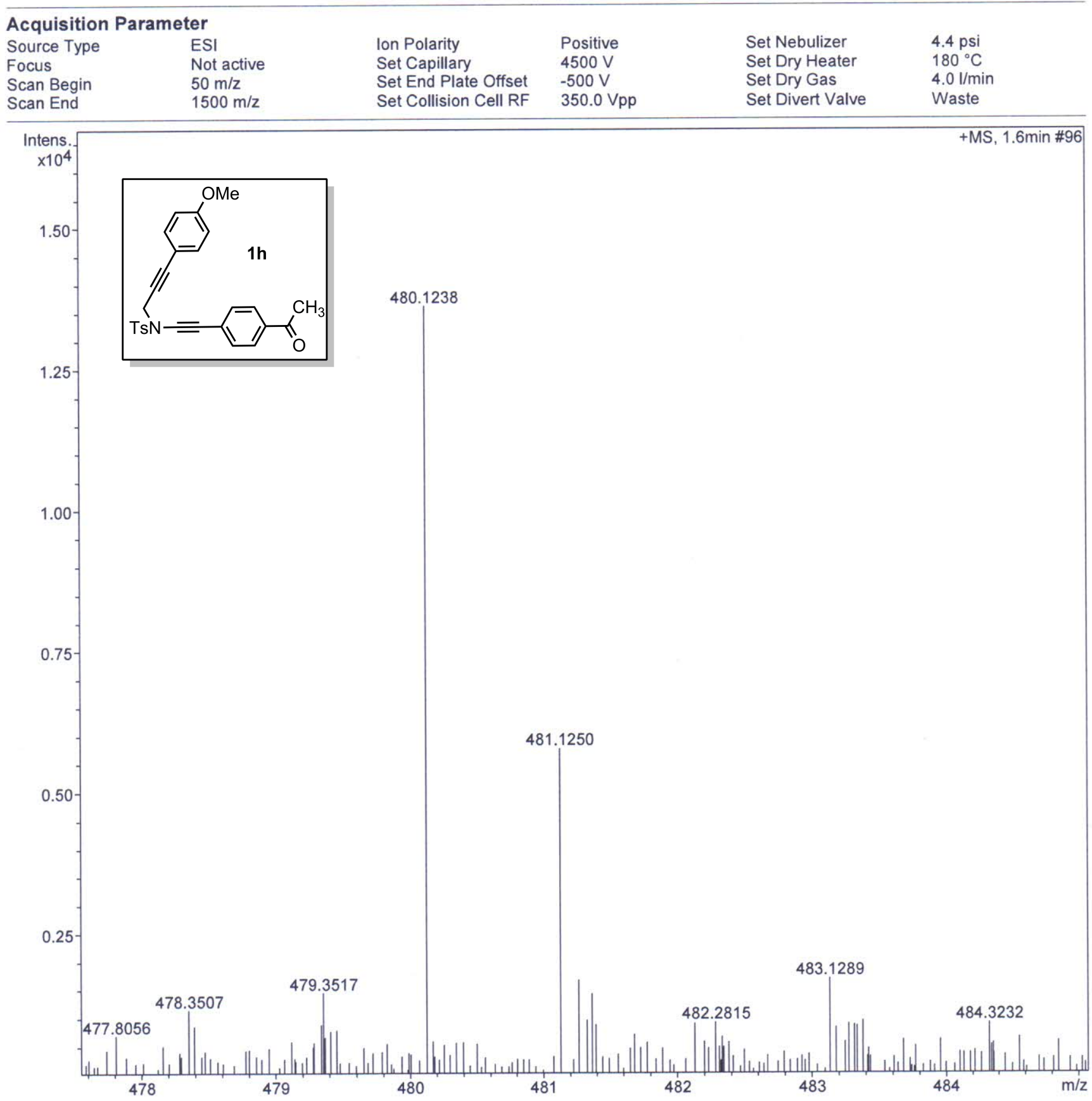




\section{BRUKER MAXIS HRMS REPORT \\ School of Chemistry \\ University of Hyderabad}

Analysis Info

Analysis Name

Method

Sample Name

Comment
D:IDatal2015IDR.AK.SAHOOIOctIRP-3-123R.d

tune_low_Pos.m

RP-3-123-MEOH
Acquisition Date

10/30/2015 12:41:39 PM

Operator Ramu Sridhar

Instrument maXis $\quad 10138$

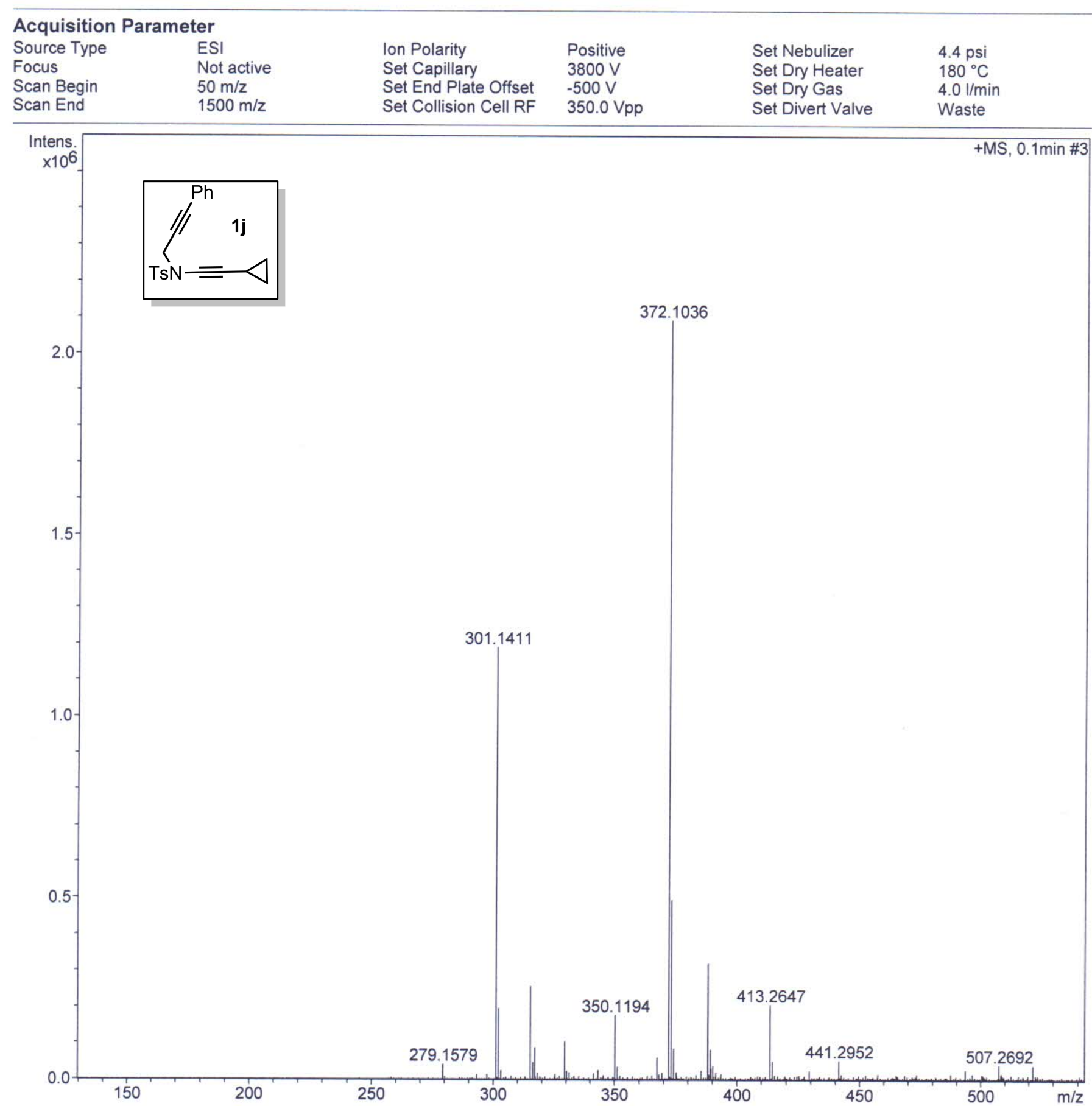




\section{BRUKER MAXIS HRMS REPORT \\ School of Chemistry \\ University of Hyderabad}

Analysis Info

Analysis Name Method

Sample Name

Comment
Acquisition Date

D:IDatal2015IDR.AK.SAHOOIOctIRP-3-112R.d

TL-P.m

RP-3-112-MEOH
10/30/2015 12:51:22 PM

Operator Ramu Sridhar

Instrument maXis

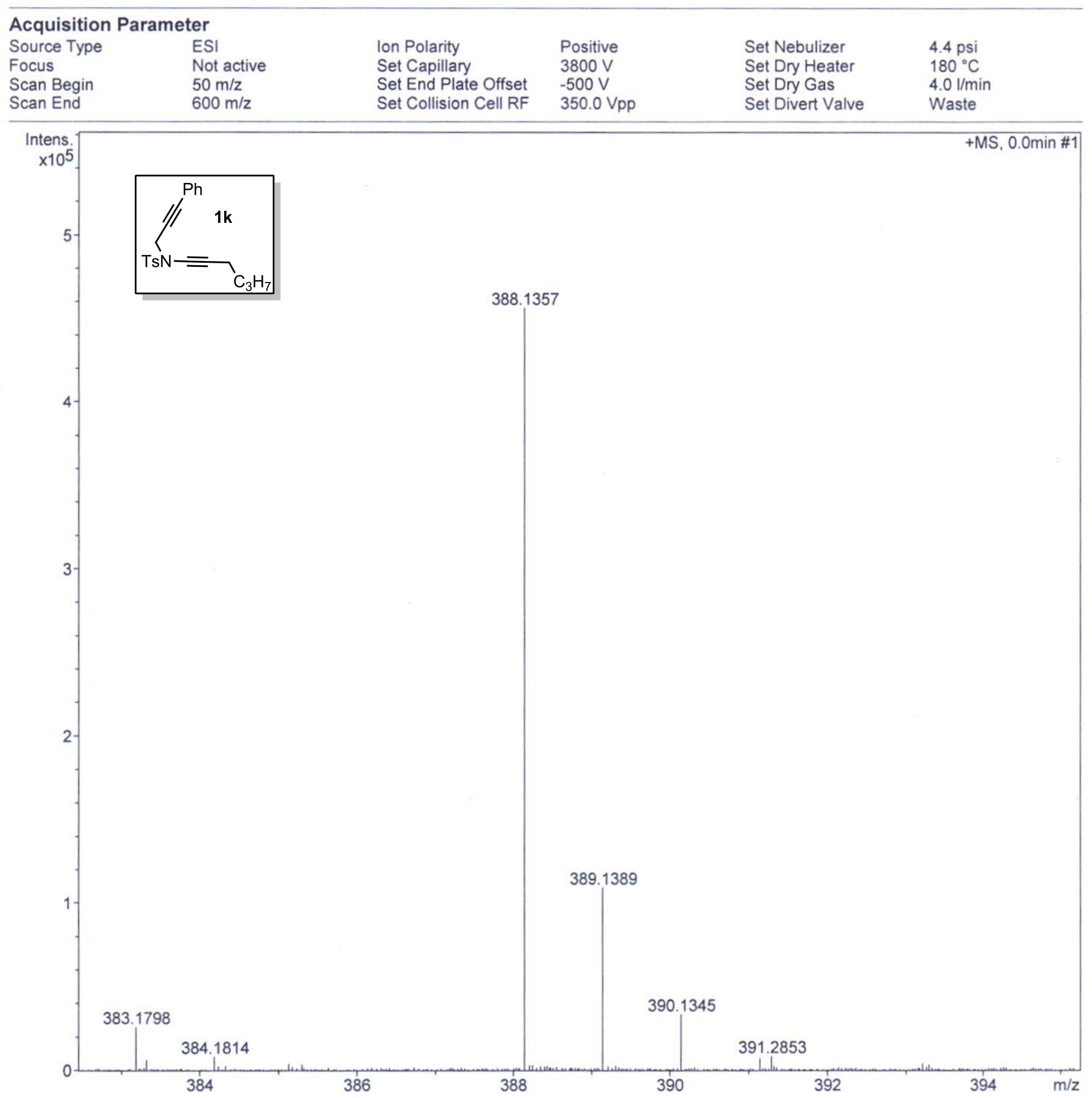




\section{UOH -SCHOOL OF CHEMISTRY -HRMS}

\section{Analysis Info}

Analysis Name Method

D:IDatal2016IDR.A.K.SAHOOIMAYIPB-10-46.d tune_low_Pos.m

PB-10-46

Sample Name
Acquisition Date

Operator

Instrument
5/25/2016 4:21:44 PM

Rajesh Vashisth maXis

Comment

\section{Ion Polarity}

Set Capillary

Set End Plate Offse

Set Collision Cell RF
Positive

$4200 \mathrm{~V}$

$-500 \mathrm{~V}$

$350.0 \mathrm{Vpp}$
Set Nebulizer

Set Dry Heater

Set Dry Gas

Set Divert Valve
$0.3 \mathrm{Bar}$

$180^{\circ} \mathrm{C}$

$4.0 \mathrm{l} / \mathrm{min}$

Waste

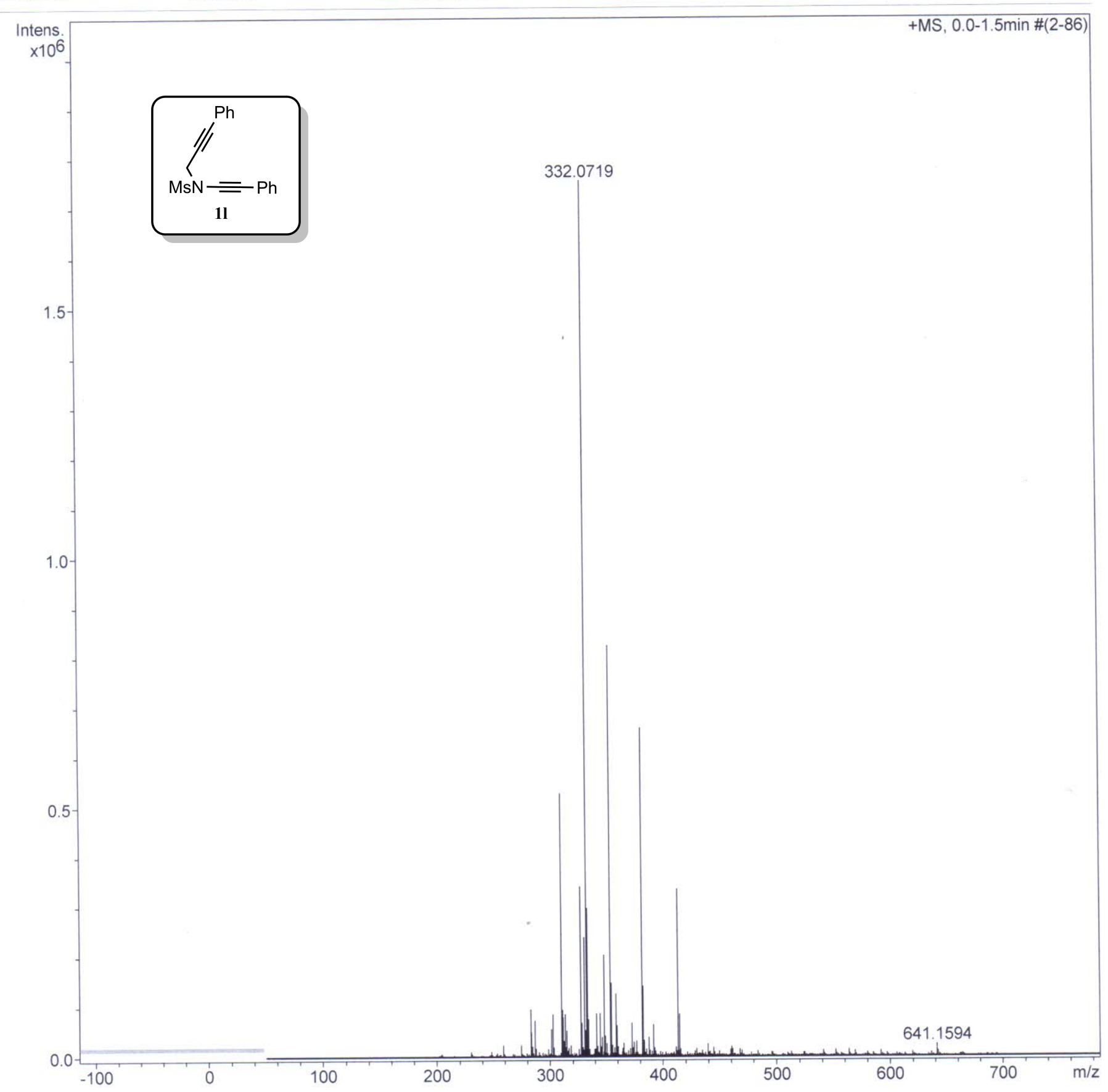




\section{BRUKER MAXIS HRMS REPORT \\ School of Chemistry \\ University of Hyderabad}

Analysis Info

Analysis Name

Method

Sample Name

Comment
D:IDatal2015IDR.AK.SAHOOIJULYYPB-4-125.d

tune_low.m

PB-4-125
Acquisition Date

7/16/2015 3:09:59 PM

Operator

Instrument
Ramu Sridhar

maXis

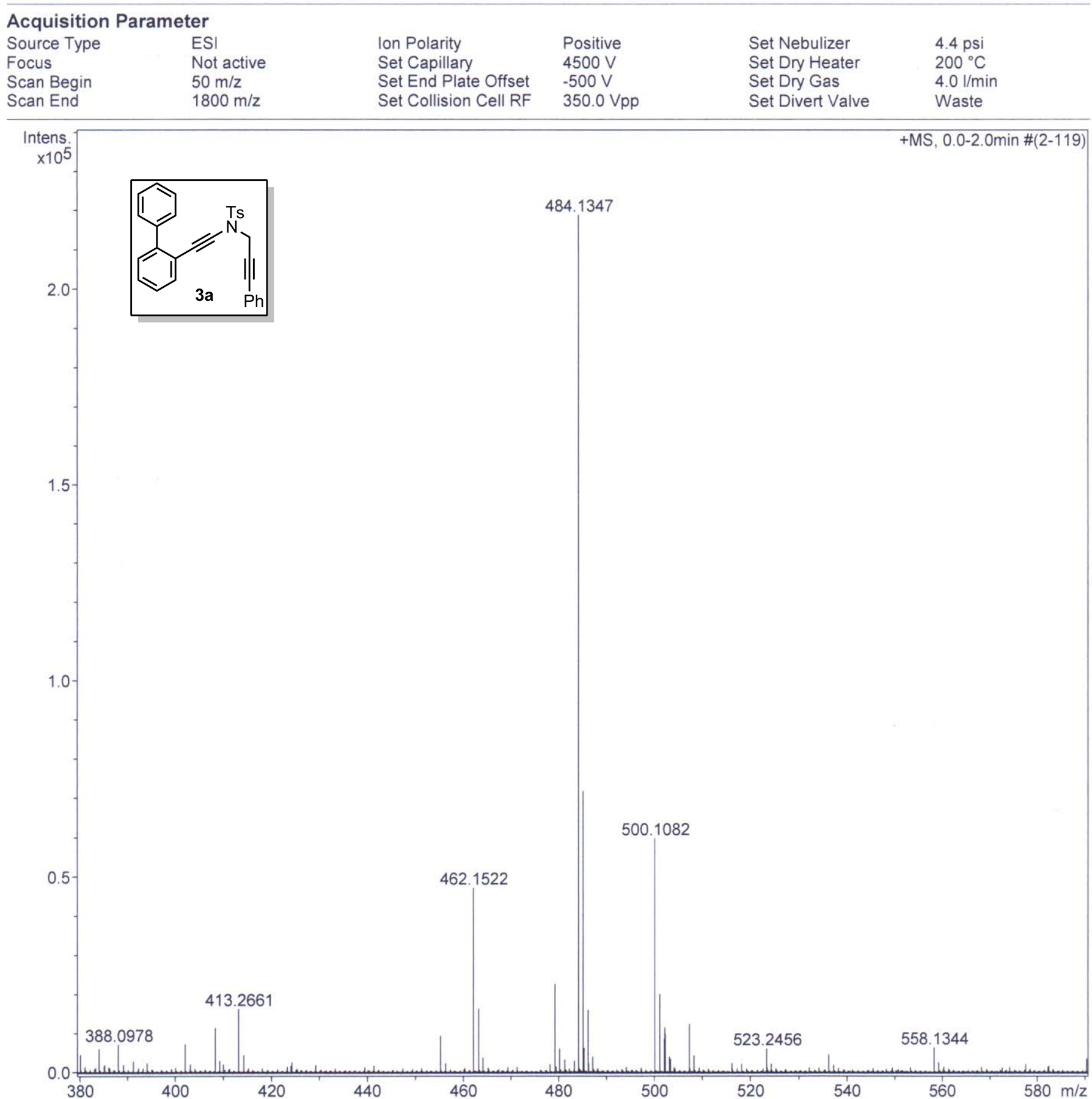




\section{BRUKER MAXIS HRMS REPORT \\ School of Chemistry \\ University of Hyderabad}

Analysis Info

Analysis Name Method

Sample Name

Comment
Acquisition Date

5/13/2015 6:49:52 PM

D:IDatal2015IDR.AK.SAHOOIMAYYPB-5-74.d tune_low_Pos-R2.m PB-5-74
Operator Instrument
Ramu Sridhar maXis 10138

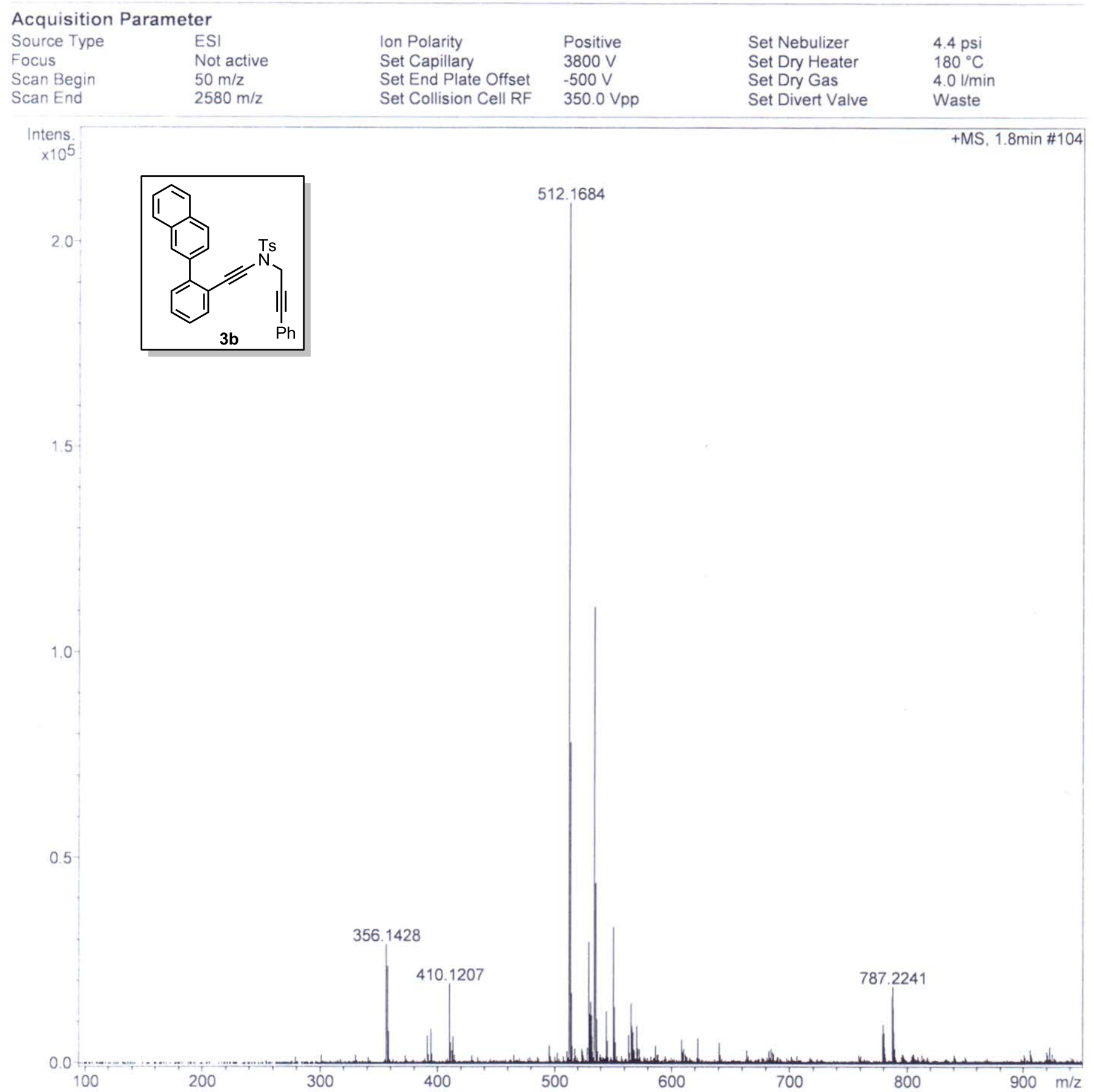




\section{BRUKER MAXIS HRMS REPORT \\ School of Chemistry \\ University of Hyderabad}

Analysis Info

Analysis Name Method

Sample Name

Comment
Acquisition Date

D:IDatal2015IDR.AK.SAHOOIMAYSn-09-117.d tune low.m

Sn-09-117-DCM-MEOH
5/15/2015 10:50:06 AM

Operator

Instrument
Ramu Sridhar maXis

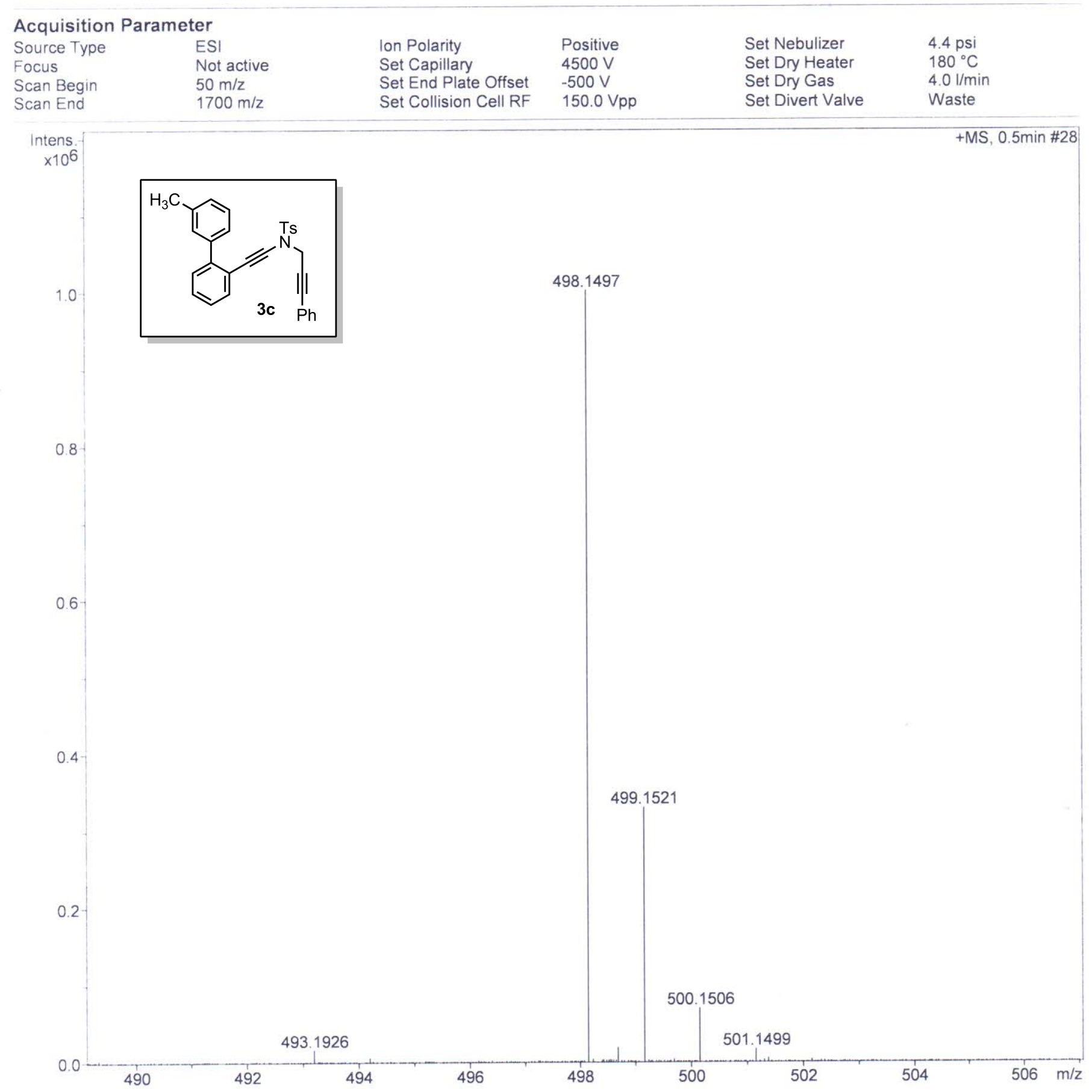




\section{BRUKER MAXIS HRMS REPORT \\ School of Chemistry \\ University of Hyderabad}

Analysis Info

Analysis Name

Method

Sample Name

Comment
Acquisition Date

D:IDatal2015IDR.AK.SAHOOIOctIPB-5-75.d

tune_low.m

PB-5-75-MEOH
Operator

Instrument
10/21/2015 4:22:07 PM

Ramu Sridhar maXis

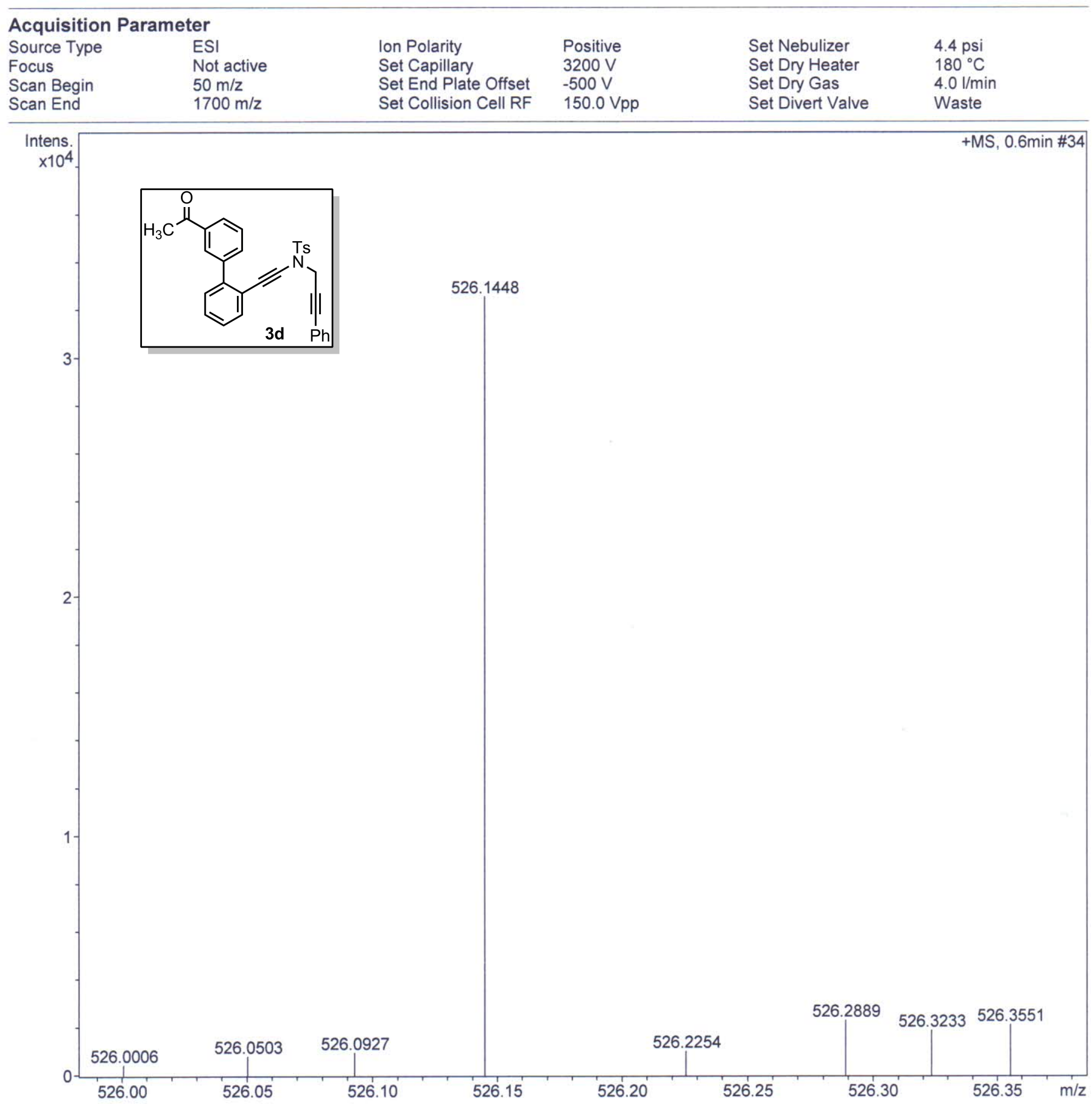




\section{BRUKER MAXIS HRMS REPORT \\ School of Chemistry \\ University of Hyderabad}

Analysis Info

Analysis Name

Method

Sample Name

Comment
D:IDatal2015IDR.AK.SAHOOIMAYIPB-A-203.d

tune_low.m

PB-A-203
Acquisition Date

5/13/2015 4:03:33 PM

Operator Ramu Sridhar

Instrument maXis

10138

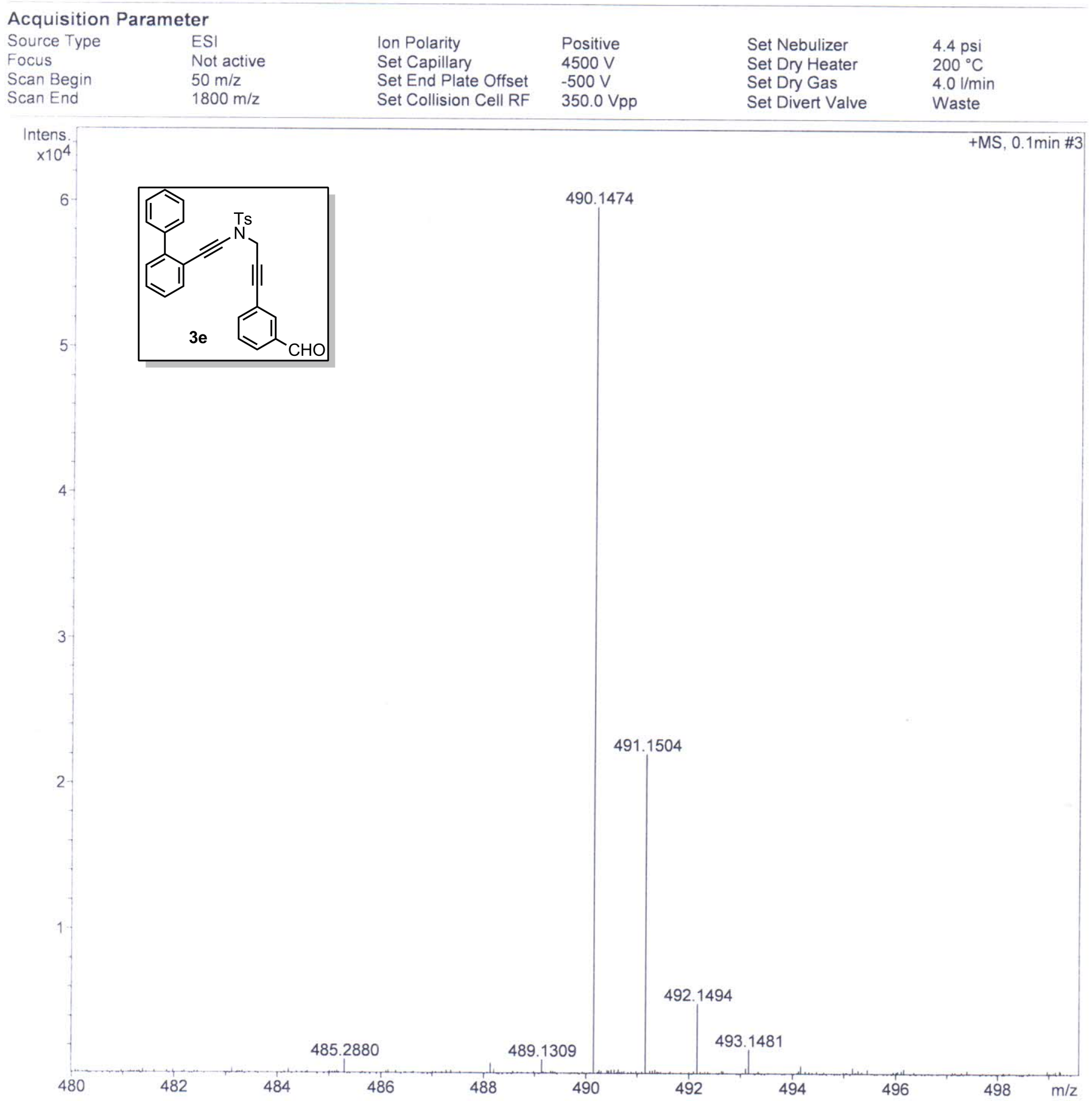




\section{BRUKER MAXIS HRMS REPORT \\ School of Chemistry \\ University of Hyderabad}

Analysis Info

Analysis Name Method

Sample Name

Comment
D:IDatal2015IDR.AK.SAHOOIFEBIPB-5-190.d tune_low_Pos-R2.m

PB-5-190
Acquisition Date

3/3/2015 5:09:22 PM

Operator

Instrument

Ramu Sridhar

maXis

10138

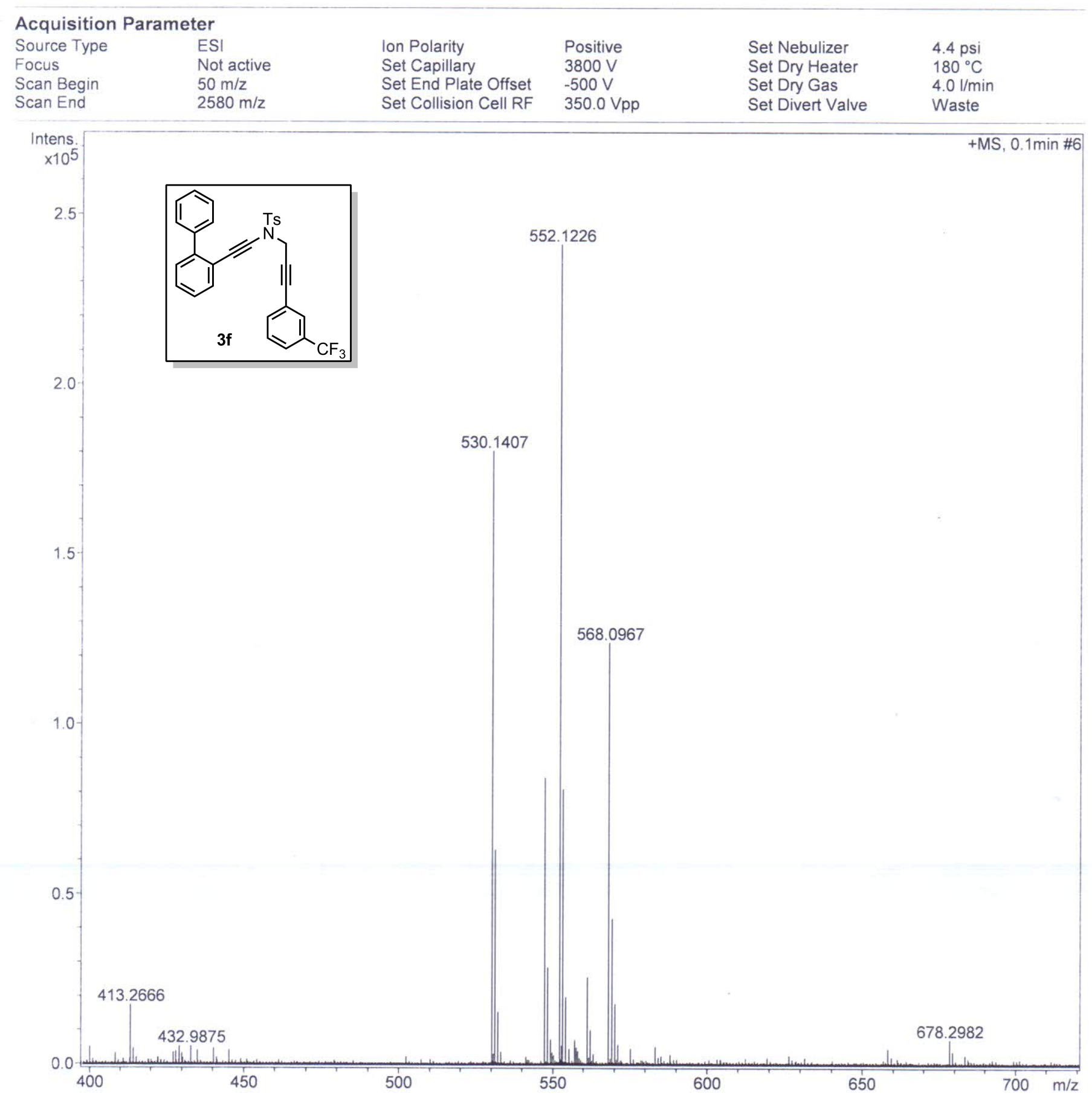




\section{BRUKER MAXIS HRMS REPORT \\ School of Chemistry \\ University of Hyderabad}

Analysis Info

Analysis Name Method

Sample Name

Comment
Acquisition Date

D:IDatal2015IDR.AK.SAHOOlaprIIPB-4-174.d tune low.m

PB-4-174-DCM-MEOH
Operator

Instrument
4/27/2015 12:01:21 PM

Ramu Sridhar maXis

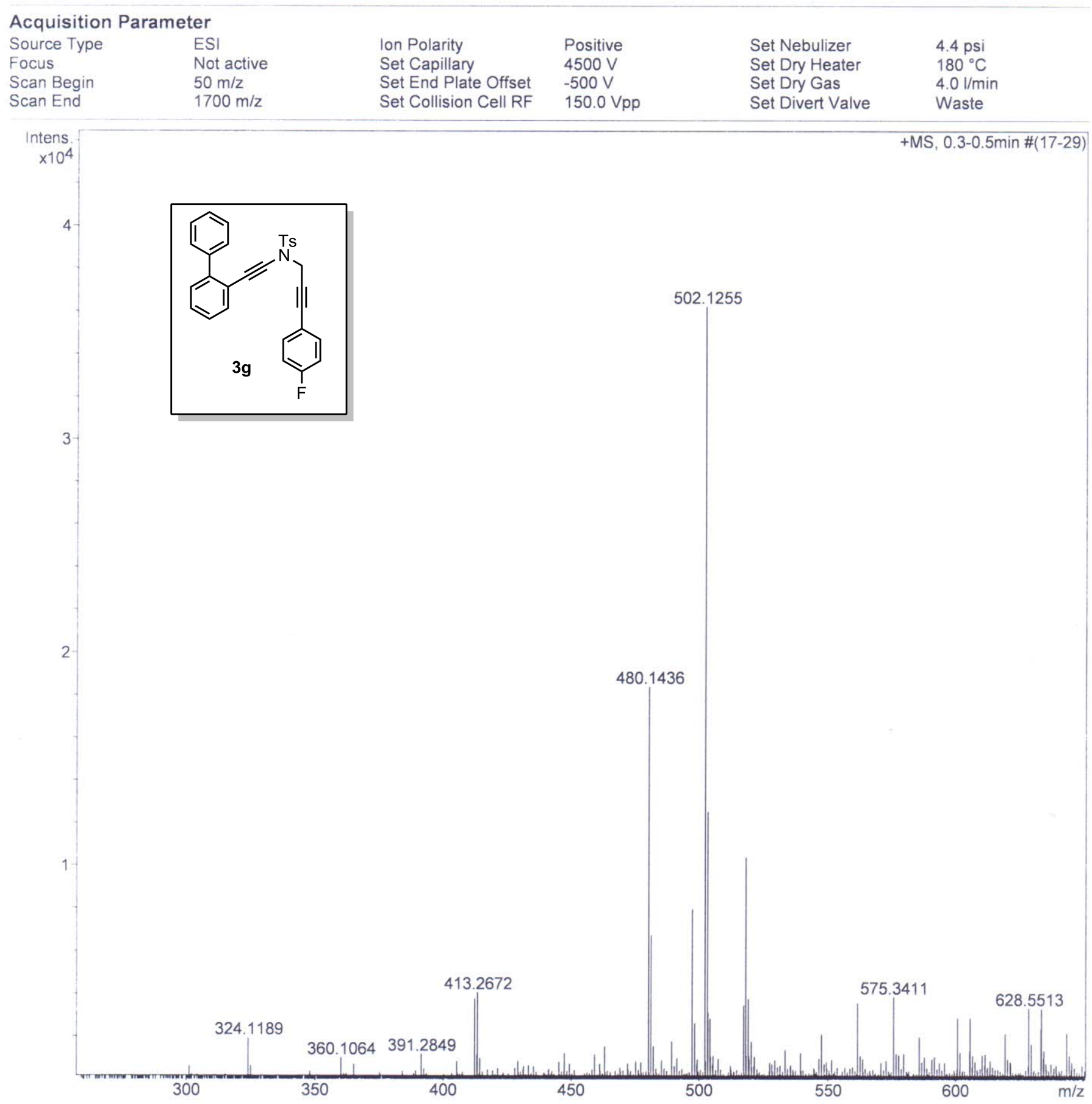




\title{
LCMS-2010A DATA REPORT SHIMADZU
}

\author{
User : Admin \\ Sample : PB-4-204 \\ Inj. Volume $\quad: 5.000$ \\ Data Name \\ : D:ILCMS-CHN DATA \\ Method Name \\ : C:LCCMSsolution।User|Methodlesi.qlm
}

MS Spectrum

Line\#:1 R.Time:0.798(Scan\#:48) Positive

MassPeaks:697 BasePeak:504.45(3023108)

RawMode:Single 0.798(48)

BG Mode:Peak Start 0.543(33)

120

$110^{\text {肄 }}$

100

90

80

70

60

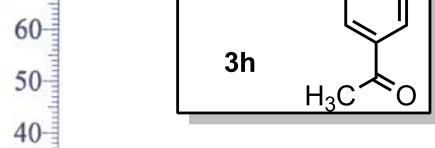

30

20

10

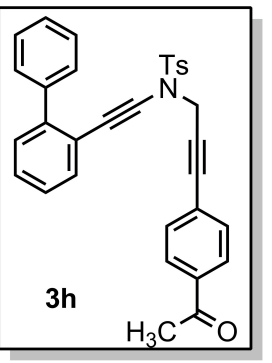

$279 \quad 394$

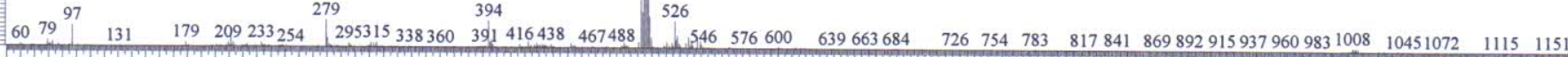

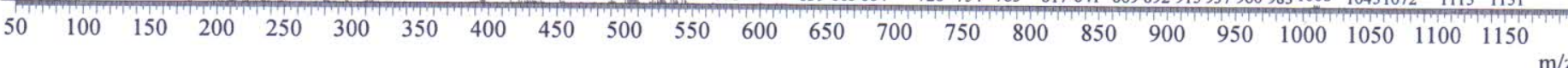

MS Peak Table

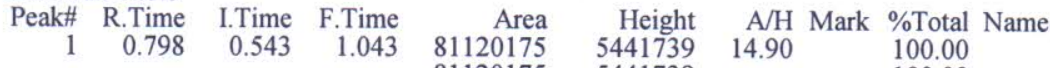

$81120175 \quad 5441739 \quad 100.00$

Base $\mathrm{m} / \mathrm{z}$ Base Int.

504.453023108 


\section{FLASH EA 1112 SERIES CHNS REPORT THERMO FINNIGAN}

Method filename:

Sample ID:

Analysis type:

Chromatogram filename:

Sample weight:
C:|Program Files $\backslash$ Thermo FinniganlEager 300 for EA1112\DATA PB-4-204 (\# 118)

UnkNown

UNK-16022016-8.dat

1.375

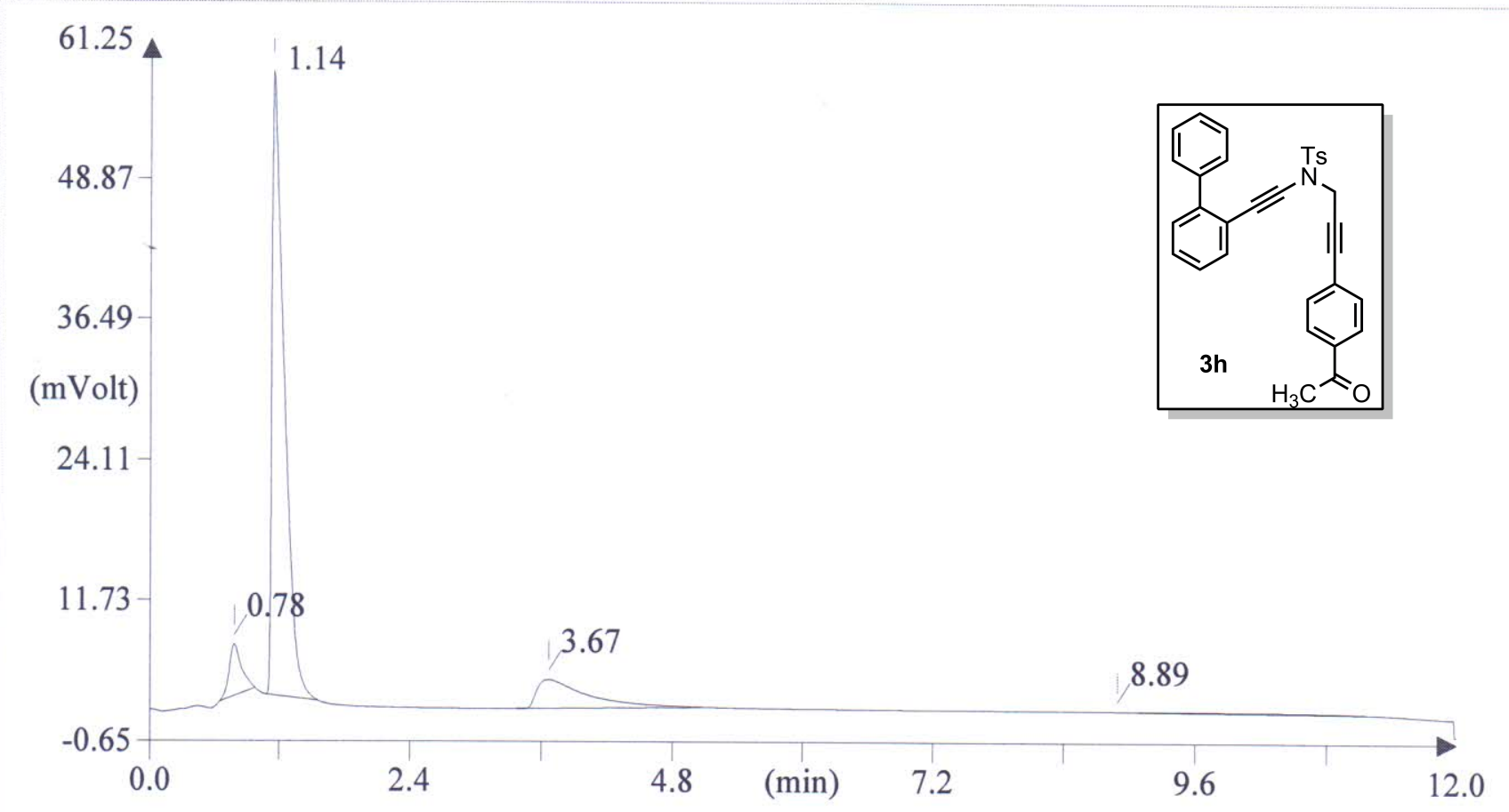

$\begin{array}{lrc}\text { Element Name } & \text { Element \% } & \text { Ret. Time } \\ \text { Nitrogen } & 2.71 & 0.78 \\ \text { Carbon } & 76.45 & 1.14 \\ \text { Hydrogen } & \text { 5. } 06 & 3.67 \\ \text { Sulphur } & \text { 6. } 29 & 8.89\end{array}$




\section{BRUKER MAXIS HRMS REPORT \\ School of Chemistry \\ University of Hyderabad}

Analysis Info

Analysis Name

Method

Sample Name

Comment
Acquisition Date

D:IDatal2015IDR.AK.SAHOOIMAYYPB-4-182.d tune_low.m

PB-4-182
Operator

Instrument
5/12/2015 3:17:18 PM

Ramu Sridhar maXis

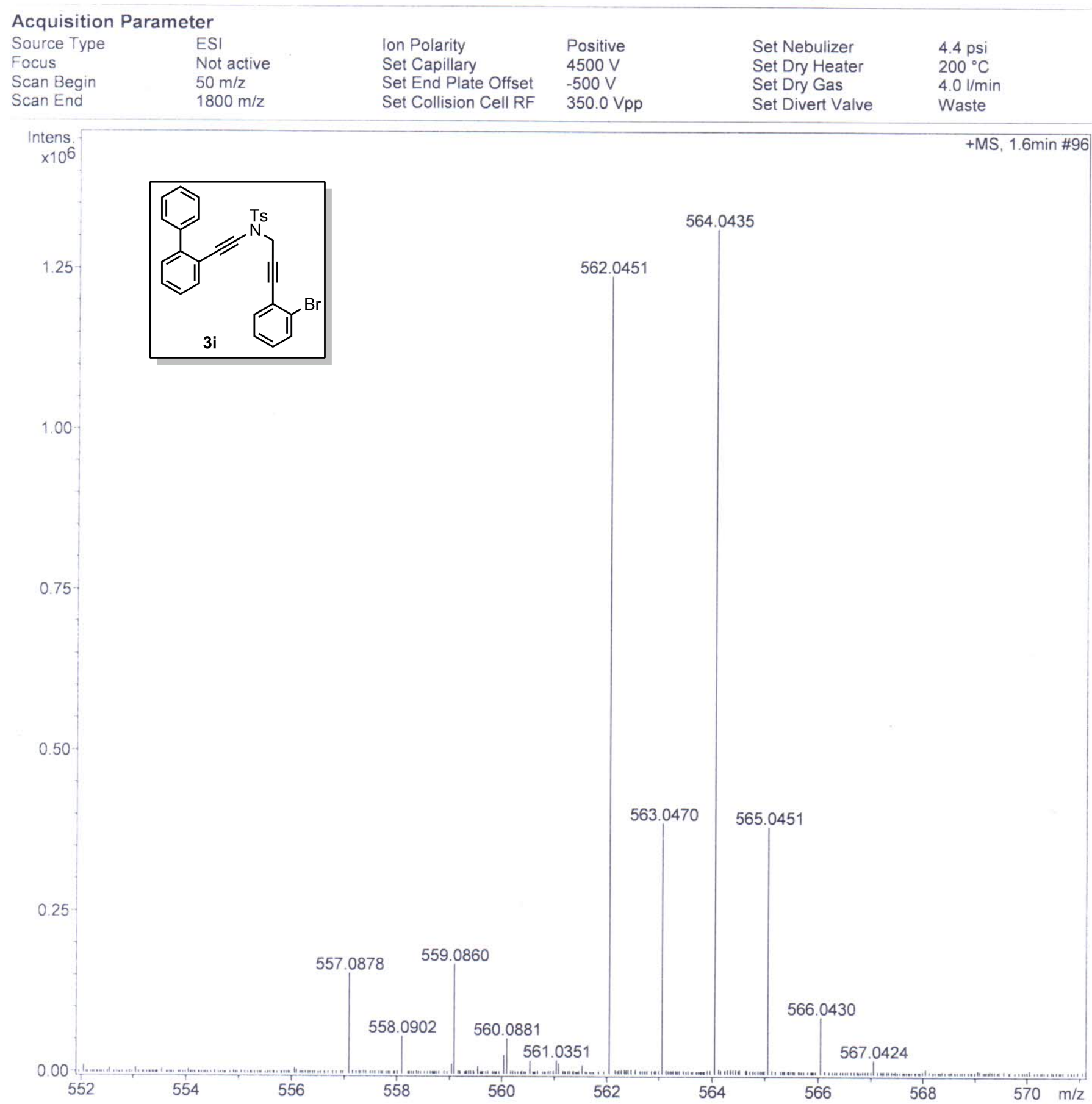




\section{UOH -SCHOOL OF CHEMISTRY -HRMS}

Analysis Info

Analysis Name Method

D:IDatal2016IDR.A.K.SAHOOIMARCHIPB-8-208.d

tune low.m

Sample Name

PB-8-208-MEOH

Comment

\section{Acquisition Parameter}

Source Type

Focus

Not active

Scan Begin

Scan End

$50 \mathrm{~m} / \mathrm{z}$

$1500 \mathrm{~m} / \mathrm{z}$

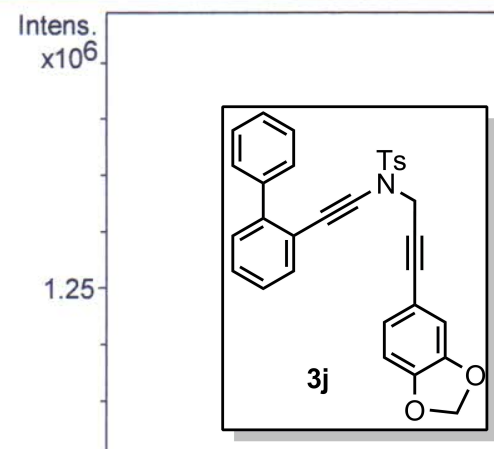

3j

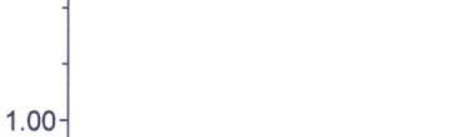

1.00

0.7

0.70

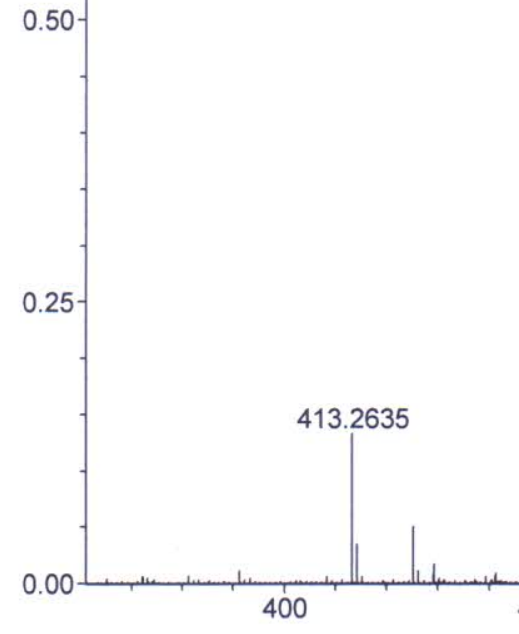

$\begin{array}{ll}\text { Ion Polarity } & \text { Positive } \\ \text { Set Capillary } & 4500 \mathrm{~V} \\ \text { Set End Plate Offset } & -500 \mathrm{~V}\end{array}$

Set End Plate Offset

Set Collision Cell RF
Acquisition Date

3/22/2016 11:05:44 AM

Operator

Instrument

Rajesh Vashisth

maXis

10138 


\section{BRUKER MAXIS HRMS REPORT \\ School of Chemistry \\ University of Hyderabad}

Analysis Info

Analysis Name

Method

Sample Name

Comment
Acquisition Date

D:IDatal2015IDR.AK.SAHOOIJULYIPB-4-176.d tune_low.m

PB-4-176
Operator

Instrument
7/16/2015 2:38:10 PM

Ramu Sridhar maXis

\section{Acquisition Parameter}

Source Type

Focus

ESI

Scan Begin

Not active

$50 \mathrm{~m} / \mathrm{z}$

$1800 \mathrm{~m} / \mathrm{z}$
Ion Polarity

Set Capillary

Set End Plate Offset

Set Collision Cell RF
Positive

$4500 \mathrm{~V}$

$-500 \mathrm{~V}$

$350.0 \mathrm{Vpp}$
Set Nebulizer $\quad 4.4 \mathrm{psi}$

Set Dry Heater $\quad 200^{\circ} \mathrm{C}$

Set Dry Gas $\quad 4.0 \mathrm{l} / \mathrm{min}$

Set Divert Valve Waste

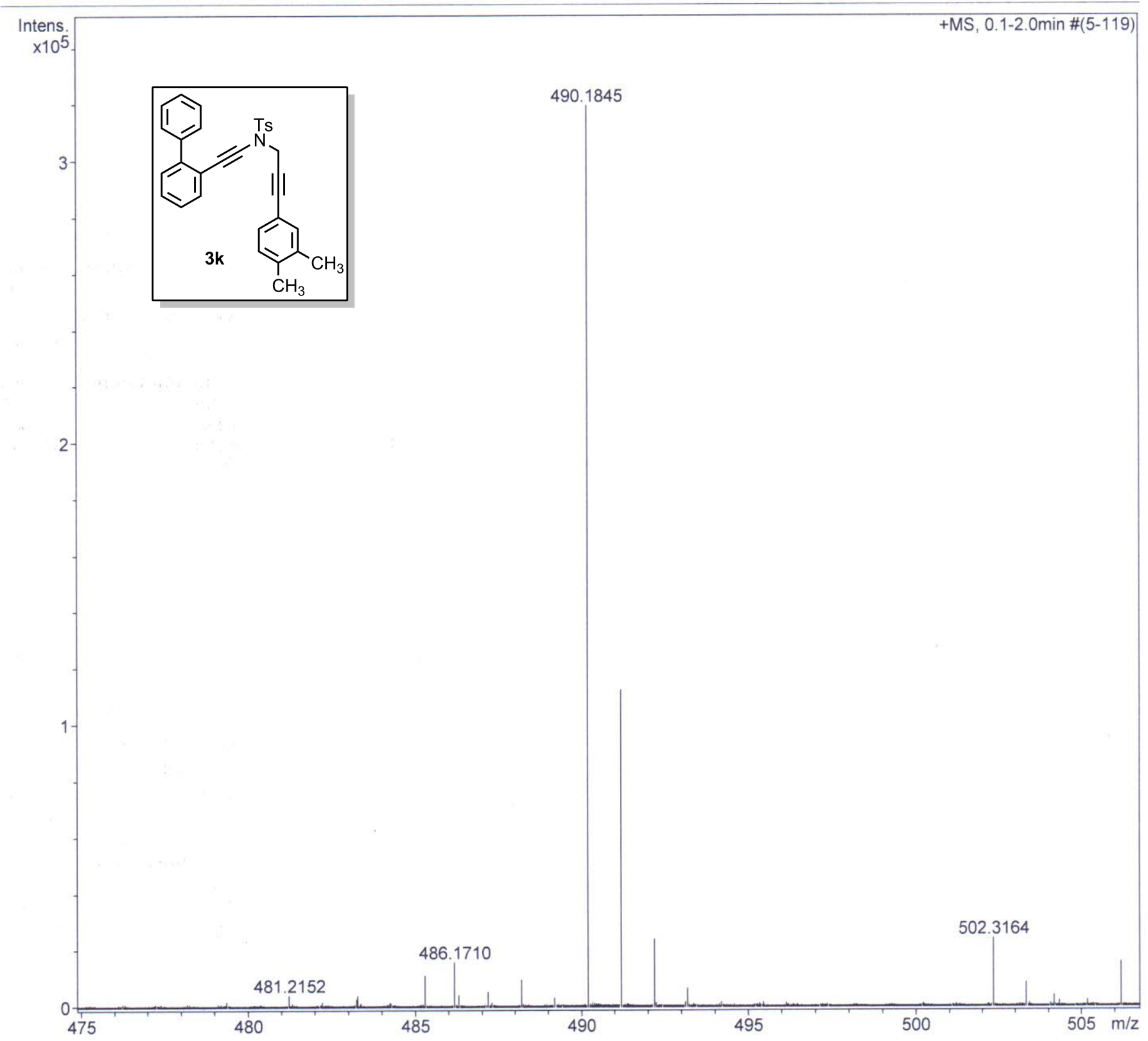




\section{BRUKER MAXIS HRMS REPORT \\ School of Chemistry \\ University of Hyderabad}

Analysis Info

Analysis Name

Method

Sample Name

Comment
Acquisition Date

D:IDatal2015IDR.AK.SAHOOIJULYSn-09-129.d tune_low_Pos-R2.m

Sn-09-129
Operator

Instrument
7/30/2015 4:02:03 PM

Ramu Sridhar maXis 10138

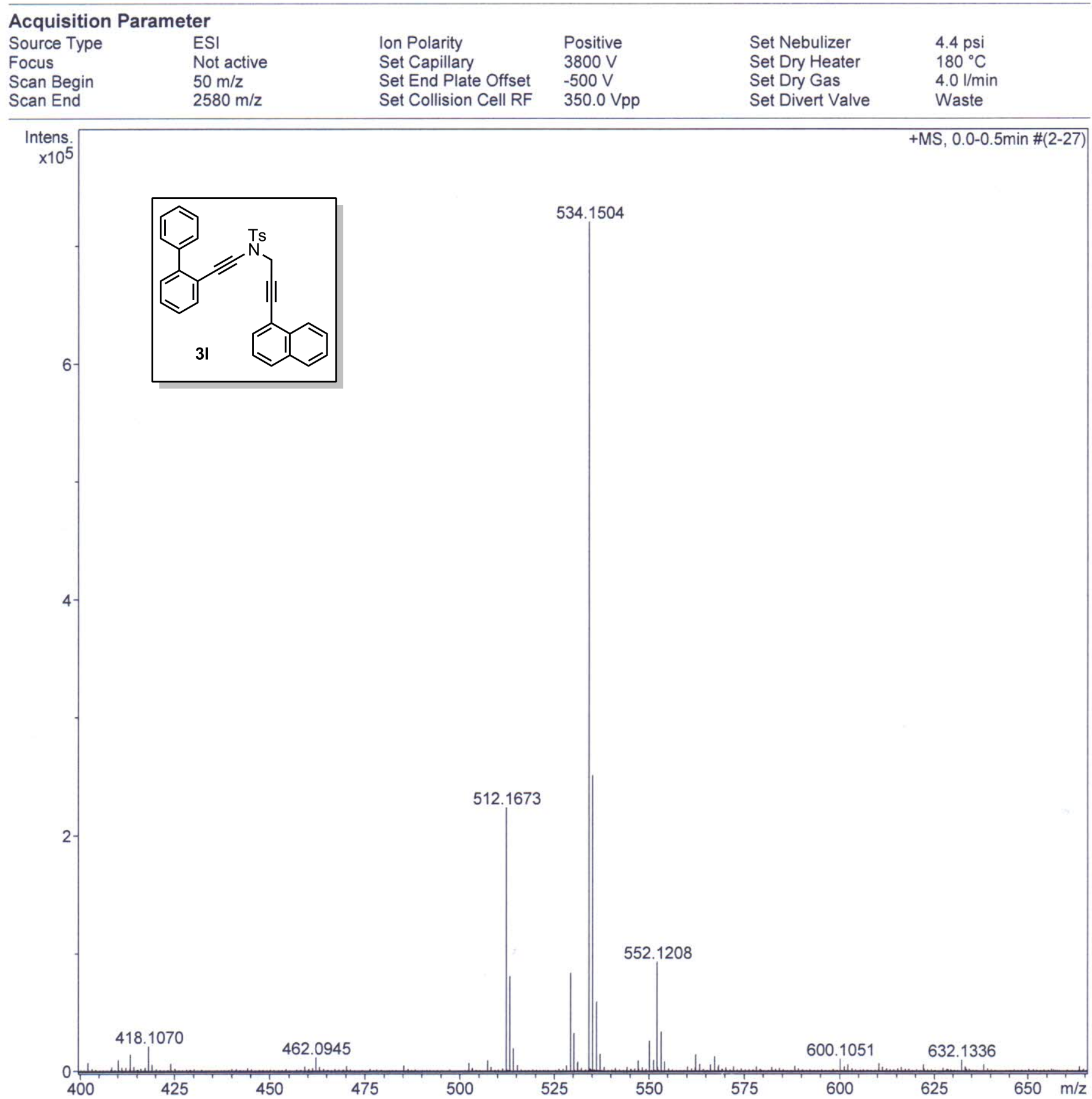




\section{UOH -SCHOOL OF CHEMISTRY -HRMS}

\section{Analysis Info}

Analysis Name

Method

Sample Name

Comment

\section{Acquisition Parameter}

Source Type

Focus

Scan Begin

ESI

Not active

$50 \mathrm{~m} / \mathrm{z}$

$1500 \mathrm{~m} / \mathrm{z}$

Scan End

Intens.

$\times 10^{6}$

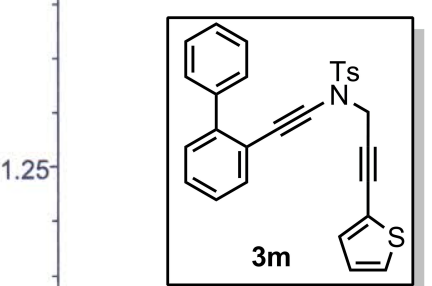

$3 m$

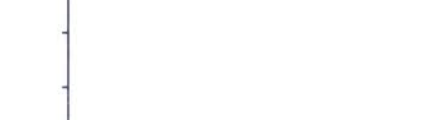

1.00

0.75 tune_low.m

PB-8-197-MEOH
Acquisition Date $\quad 3 / 22 / 2016$ 10:40:15 AM

Operator Rajesh Vashisth

Instrument maXis

10138
Ion Polarity

Set Capillary

Set End Plate Offset

Set Collision Cell RF

Positive

$4500 \mathrm{~V}$

$-500 \mathrm{~V}$

$350.0 \mathrm{Vpp}$
Set Nebulizer

Set Dry Heater

Set Dry Gas

Set Divert Valve
$0.3 \mathrm{Bar}$

$180^{\circ} \mathrm{C}$

$4.0 \mathrm{l} / \mathrm{min}$

Waste

+MS, 1.1-1.3min \#(65-79)

\subsection{6}
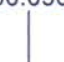

491.0930

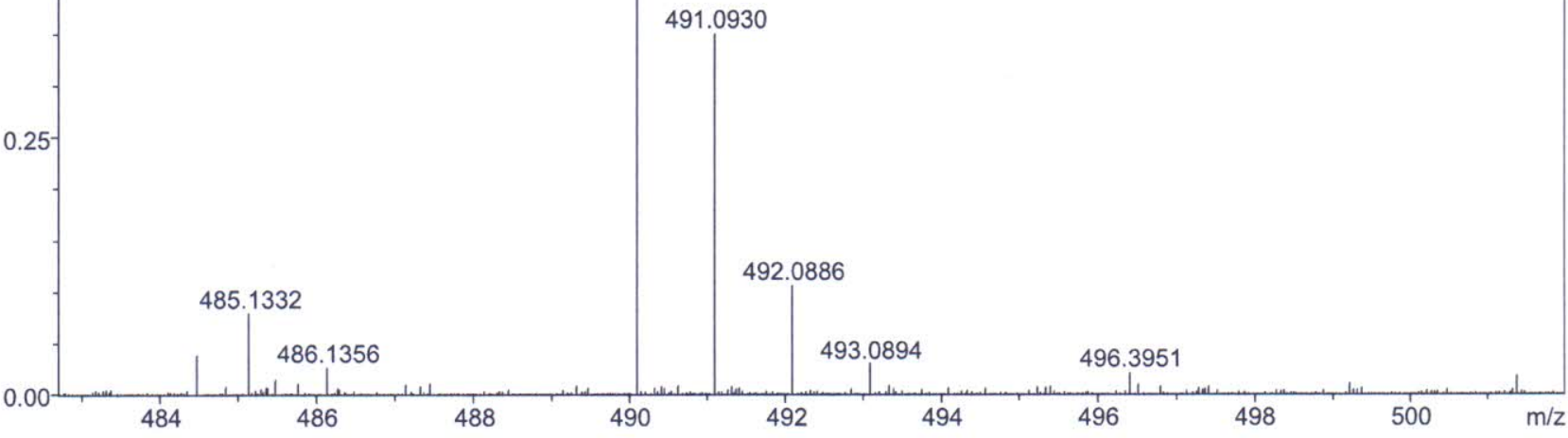




\section{UOH -SCHOOL OF CHEMISTRY -HRMS}

\section{Analysis Info}

Analysis Name Method

Sample Name

Comment
D:IDatal2016IDR.A.K.SAHOOIMARCHIPB-8-196.d

tune low.m

PB-8-196-MEOH
Acquisition Date $\quad 3 / 21 / 2016$ 12:39:00 PM

Operator Rajesh Vashisth

Instrument maXis

10138

Acquisition Parameter

Source Type

ESI

Focus

Scan Begin

Not active

$50 \mathrm{~m} / \mathrm{z}$

Set Capillary

$4500 \mathrm{~V}$

Scan End

$1500 \mathrm{~m} / \mathrm{z}$

Set End Plate Offset

Set Collision Cell RF

$-500 \mathrm{~V}$

$350.0 \mathrm{Vpp}$

Set Nebulizer

Set Dry Heater

Set Dry Gas

Set Divert Valve

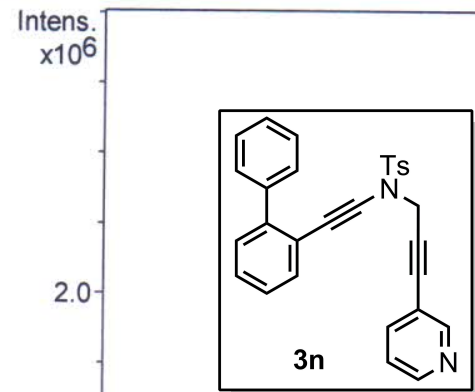

$3 n$

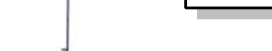

.

1.5

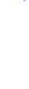
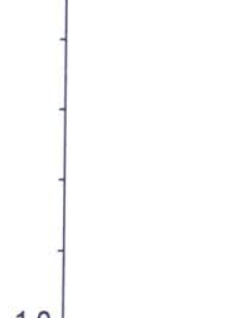

.

$-$

1

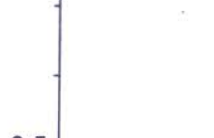

0.5

482 


\section{BRUKER MAXIS HRMS REPORT \\ School of Chemistry \\ University of Hyderabad}

Analysis Info

Analysis Name

Method

Sample Name

Comment
Acquisition Date 3/3/2015 4:00:37 PM

D:IDatal2015IDR.AK.SAHOOIFEBIPB-5-189.d

tune_low_Pos-R2.m

PB-5-188

Operator

Instrument

Ramu Sridhar

maXis

10138

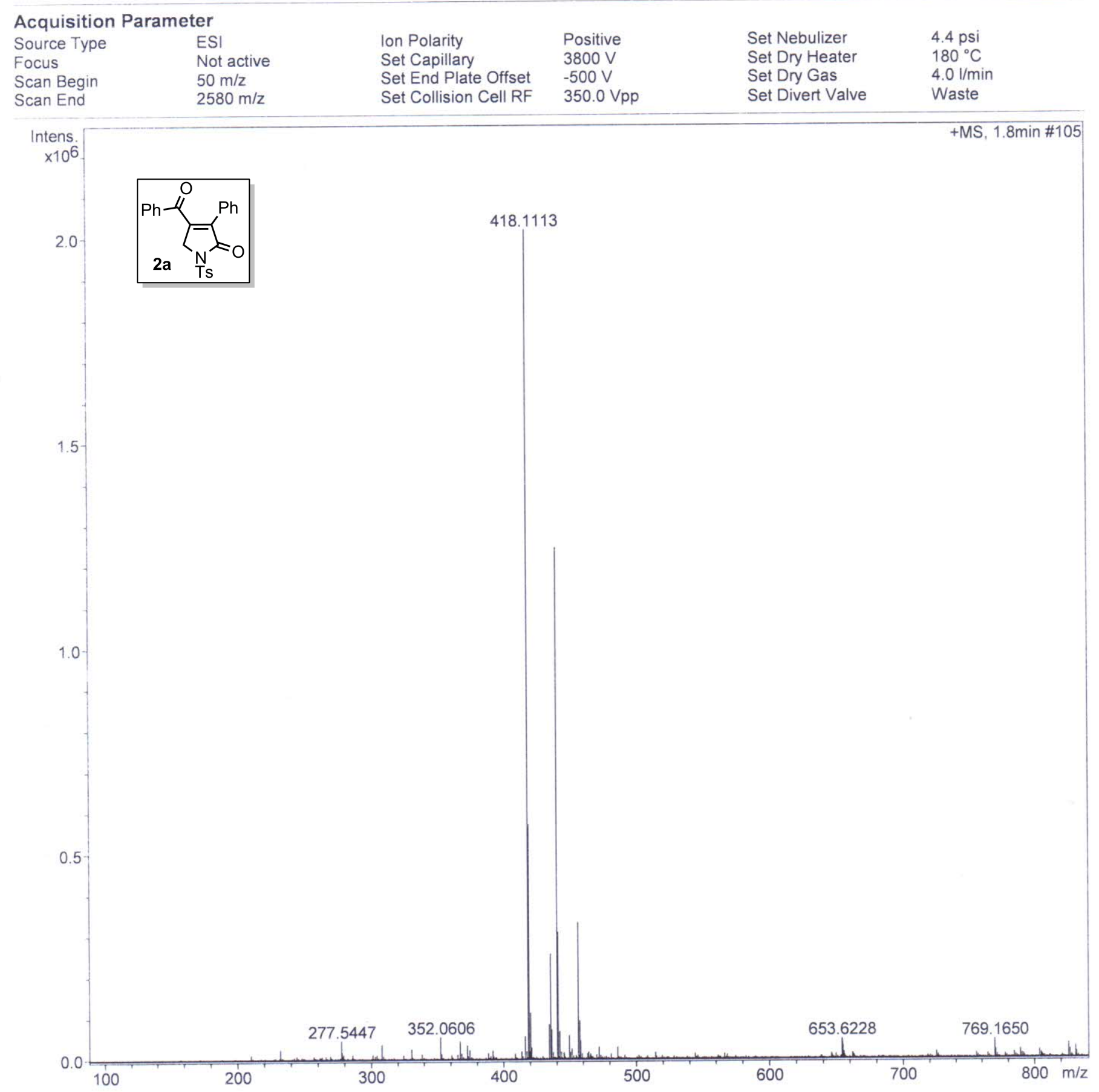




\section{BRUKER MAXIS HRMS REPORT \\ School of Chemistry \\ University of Hyderabad}

Analysis Info

Analysis Name

Method

Sample Name

Comment
Acquisition Date

2/20/2015 1:19:20 PM

D:IDatal2015IDR.AK.SAHOOIFEBIPB-6-16.d

tune_low_Pos.m

PB-6-16-MEOH
Operator Ramu Sridhar

Instrument maXis

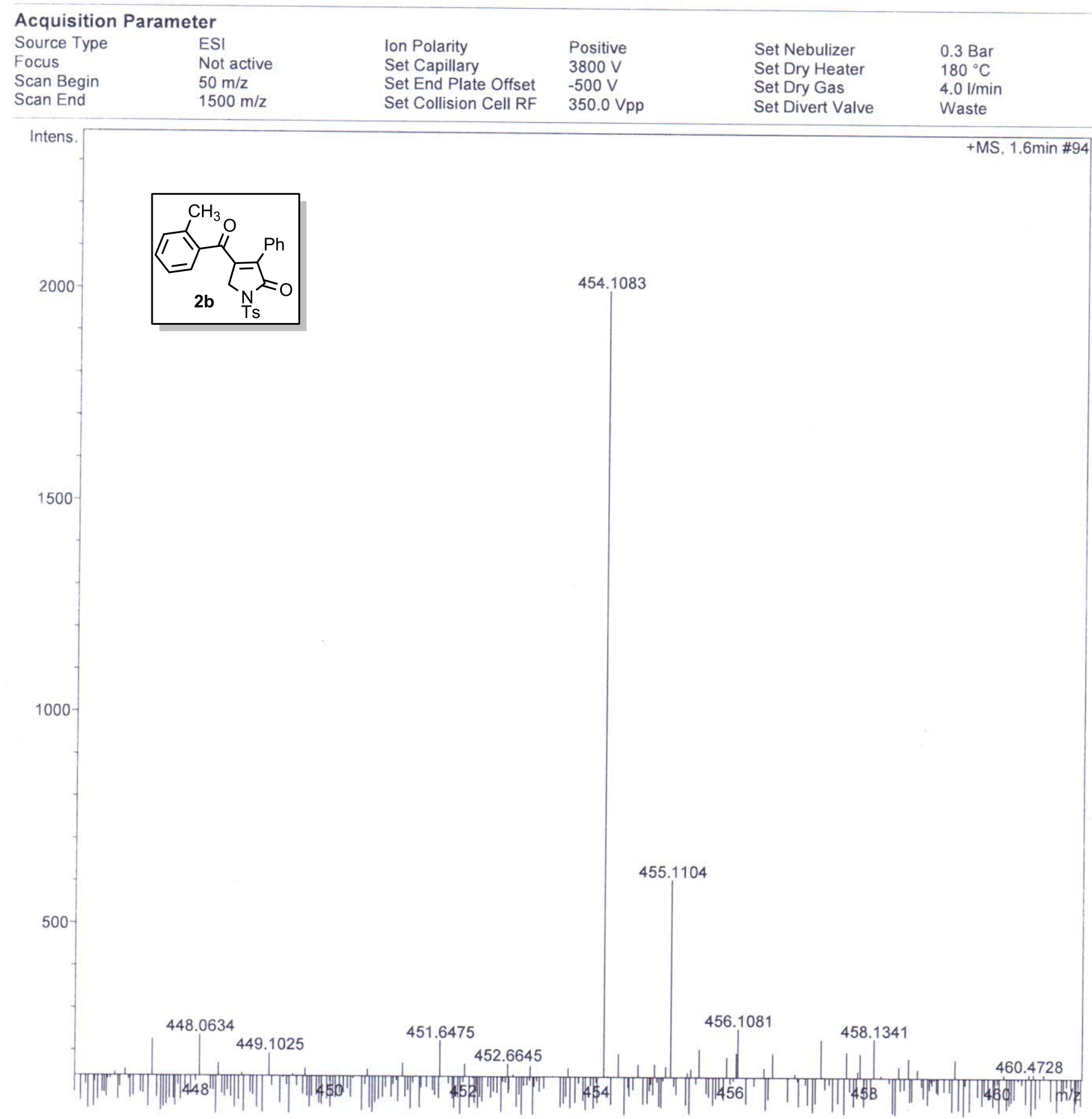




\section{BRUKER MAXIS HRMS REPORT \\ School of Chemistry \\ University of Hyderabad}

Analysis Info

Analysis Name Method

Sample Name

Comment
D:IDatal2015IDR.AK.SAHOOIMARIPB-6-32.d tune_low_Pos-R2.m

PB-6-32
Acquisition Date

3/24/2015 3:16:57 PM

Operator

Instrument
Ramu Sridhar maXis

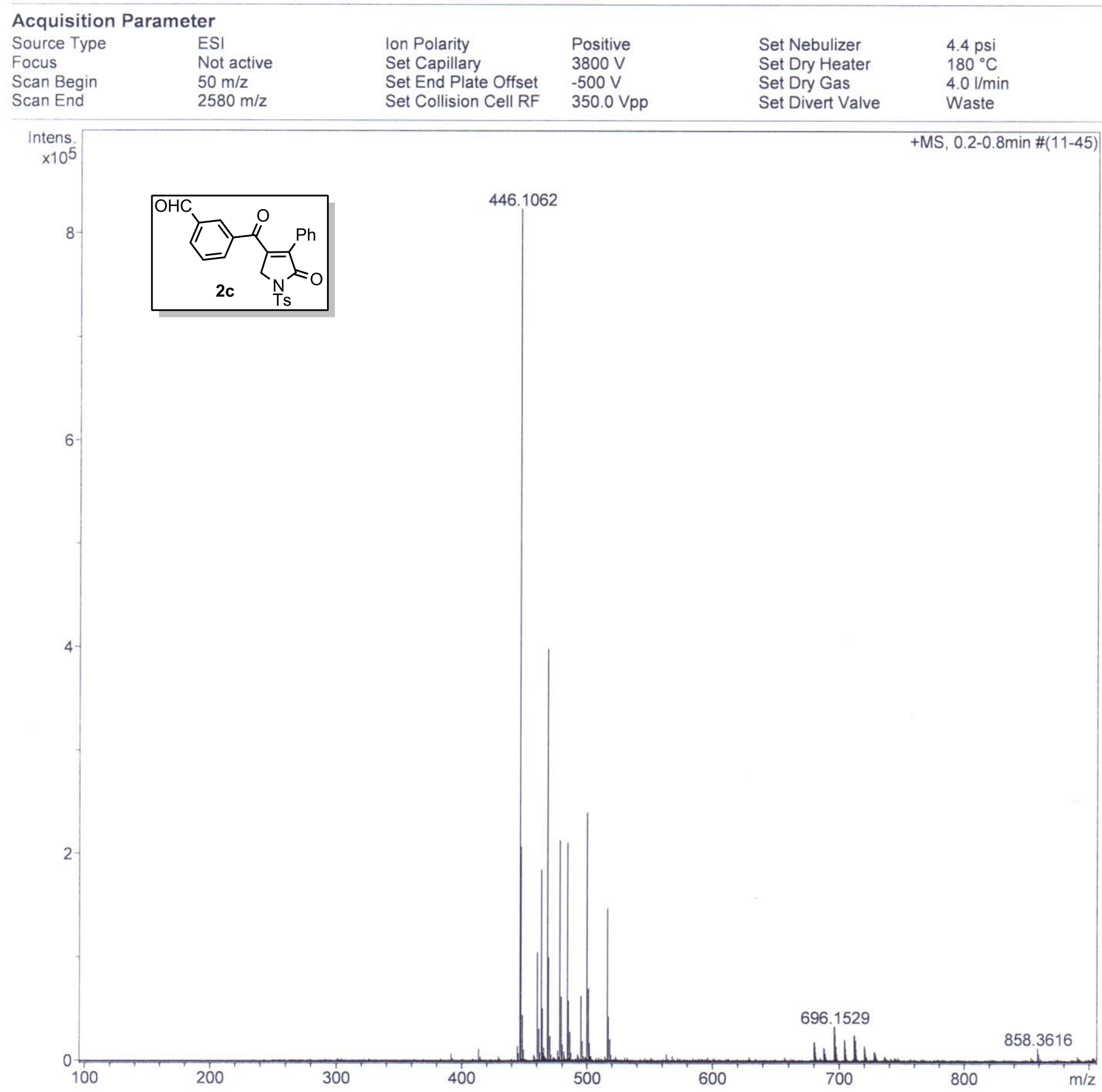




\section{BRUKER MAXIS HRMS REPORT \\ School of Chemistry \\ University of Hyderabad}

Analysis Info

Analysis Name

Method

Sample Name

Comment
D:IDatal2015IDR.AK.SAHOOIMARIPB-6-33.d

tune_low.m

PB-6-33
Acquisition Date

3/24/2015 2:56:22 PM

Operator Ramu Sridhar

Instrument maXis

\section{Acquisition Parameter}

Source Type

Focus

Scan Begin

ESI

Not active

Scan End

$50 \mathrm{~m} / \mathrm{z}$

$1800 \mathrm{~m} / \mathrm{z}$

$\begin{array}{ll}\text { Ion Polarity } & \text { Positive } \\ \text { Set Capillary } & 4500 \mathrm{~V} \\ \text { Set End Plate Offset } & -500 \mathrm{~V} \\ \text { Set Collision Cell RF } & 350.0 \mathrm{Vpp}\end{array}$

Set Nebulizer

Set Dry Heater

$4.4 \mathrm{psi}$

Set Dry Gas

Set Collision Cell RF

$350.0 \mathrm{Vpp}$

Set Divert Valve

$4.0 \mathrm{l} / \mathrm{min}$

Waste

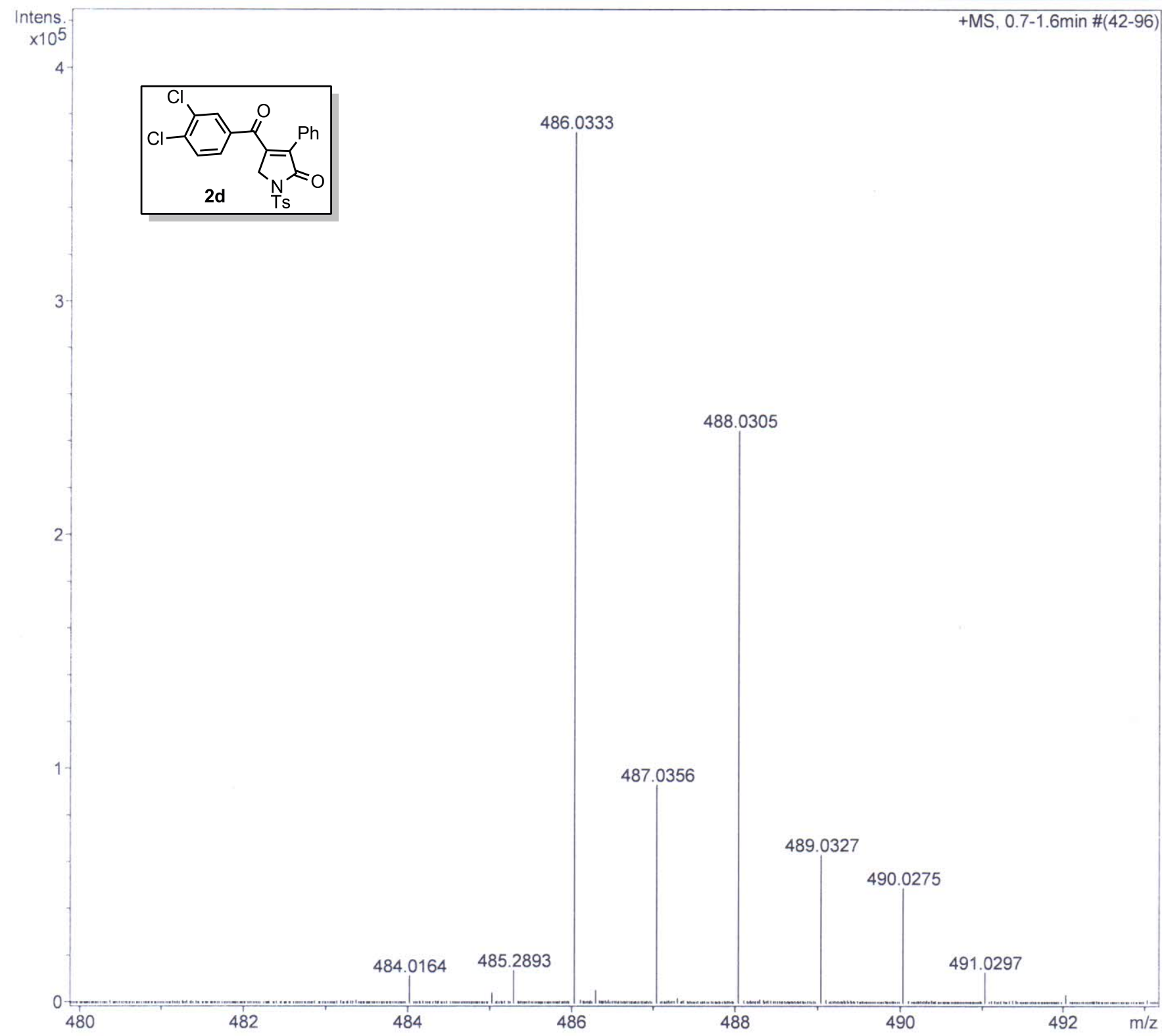




\section{BRUKER MAXIS HRMS REPORT \\ School of Chemistry University of Hyderabad}

\section{Analysis Info}

Analysis Name Method

Sample Name

Comment
Acquisition Date

D:IDatal2015IDR.AK.SAHOOINOVRP-3-134.d tune_low_Pos.m

RP-3-134
11/5/2015 3:08:56 PM

Operator Ramu Sridhar

Instrument maXis

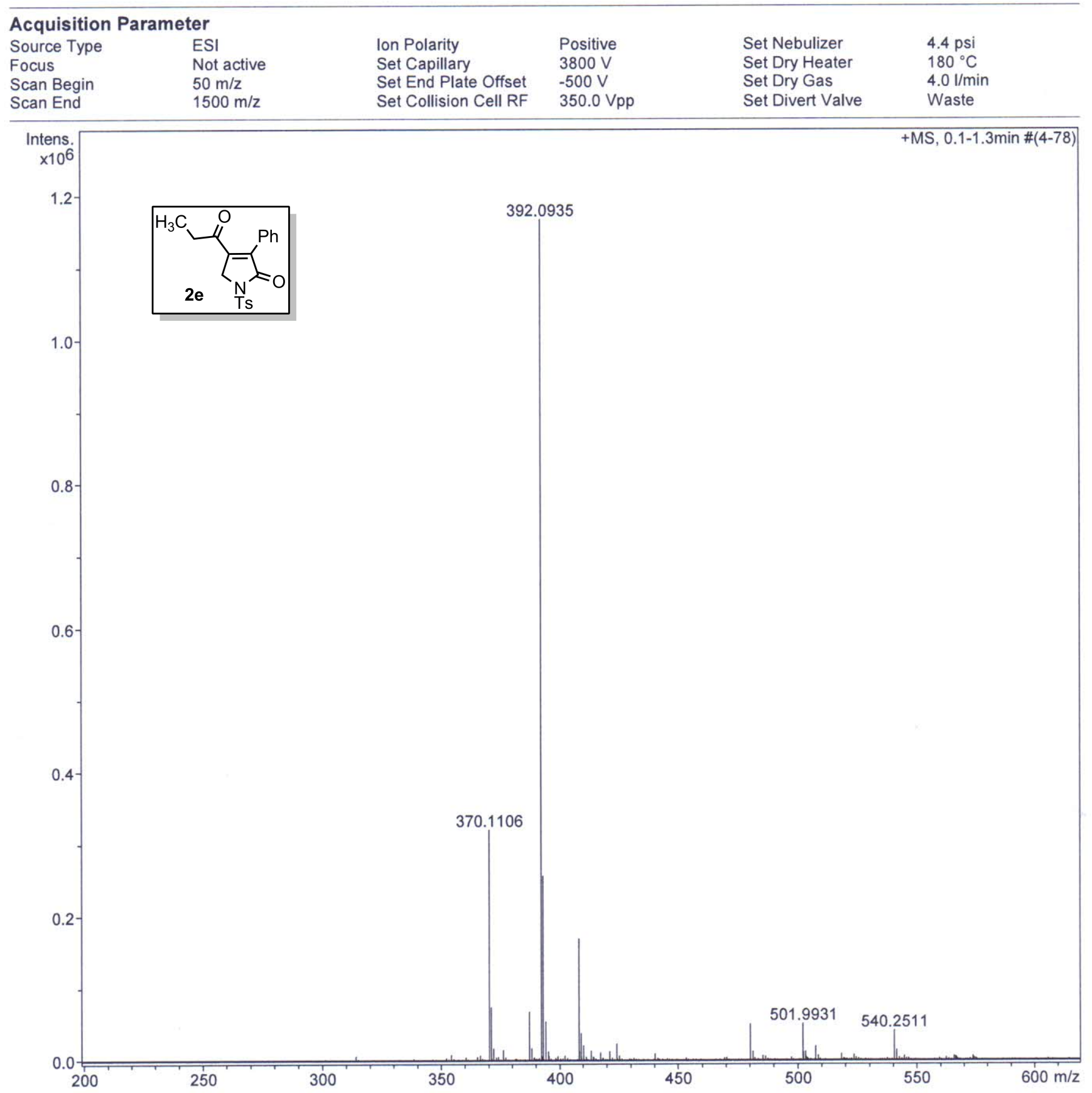




\section{BRUKER MAXIS HRMS REPORT \\ School of Chemistry \\ University of Hyderabad}

Analysis Info

Analysis Name

Method

Sample Name

Comment
Acquisition Date

D:IDatal2015IDR.AK.SAHOOIOctIPB-8-71R.d tune_low_Pos-R2.m

PB-8-71-MEOH
10/19/2015 3:02:04 PM

Operator Ramu Sridhar

Instrument

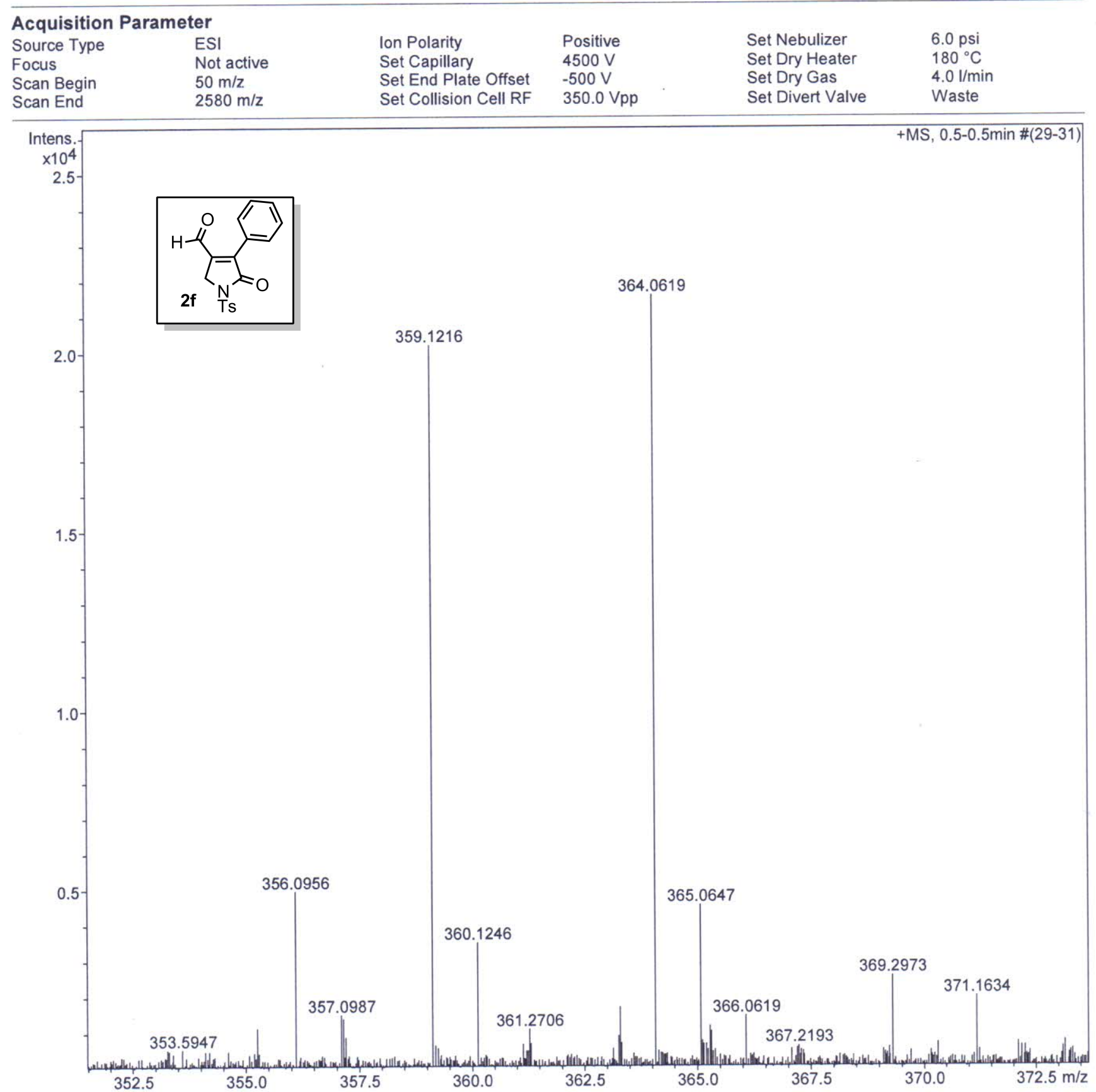




\section{BRUKER MAXIS HRMS REPORT \\ School of Chemistry \\ University of Hyderabad}

Analysis Info

Analysis Name

Method

Sample Name

Comment
D:IDatal2015IDR.AK.SAHOOINOMRP-3-143.d

tune_low_Pos.m

RP-3-143
Acquisition Date

11/5/2015 3:01:54 PM

Operator Ramu Sridhar

Instrument maXis 10138

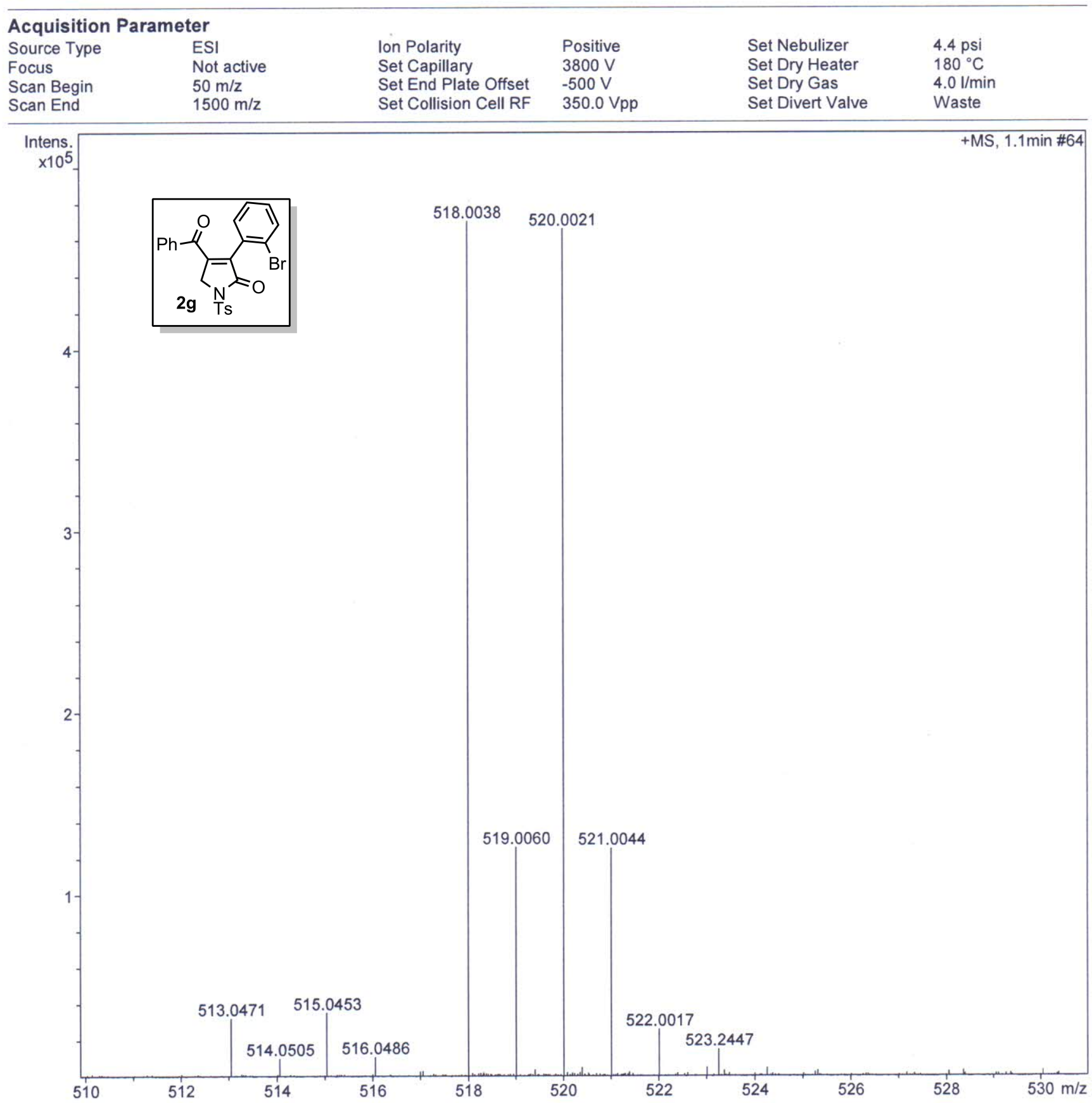




\section{BRUKER MAXIS HRMS REPORT \\ School of Chemistry \\ University of Hyderabad}

Analysis Info

Analysis Name

Method

Sample Name

D:IDatal2015IDR.AK.SAHOOIDECIRP-3-166R2.d

TL-P.m

RP-3-166-MEOH
Acquisition Date

12/3/2015 12:35:20 PM

Operator

Instrument

Ramu Sridhar maXis

\section{Comment}

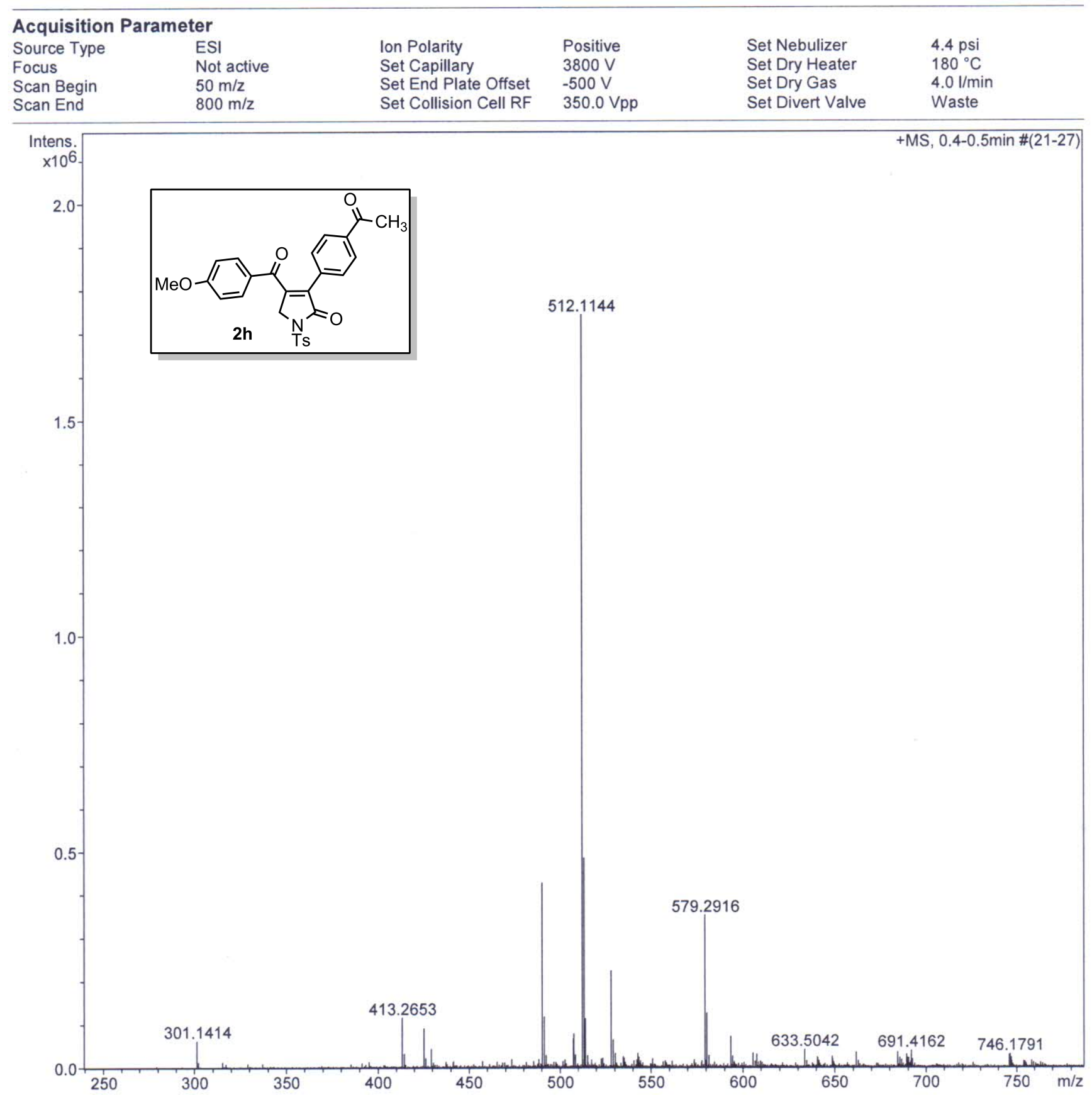




\section{BRUKER MAXIS HRMS REPORT \\ School of Chemistry \\ University of Hyderabad}

Analysis Info

Analysis Name

Method

Sample Name

Comment
D:IDatal2015IDR.AK.SAHOOINOVRP-3--122.d

tune low.m

RP-3-122
Acquisition Date

11/5/2015 2:17:29 PM

Operator

Instrument
Ramu Sridhar maXis

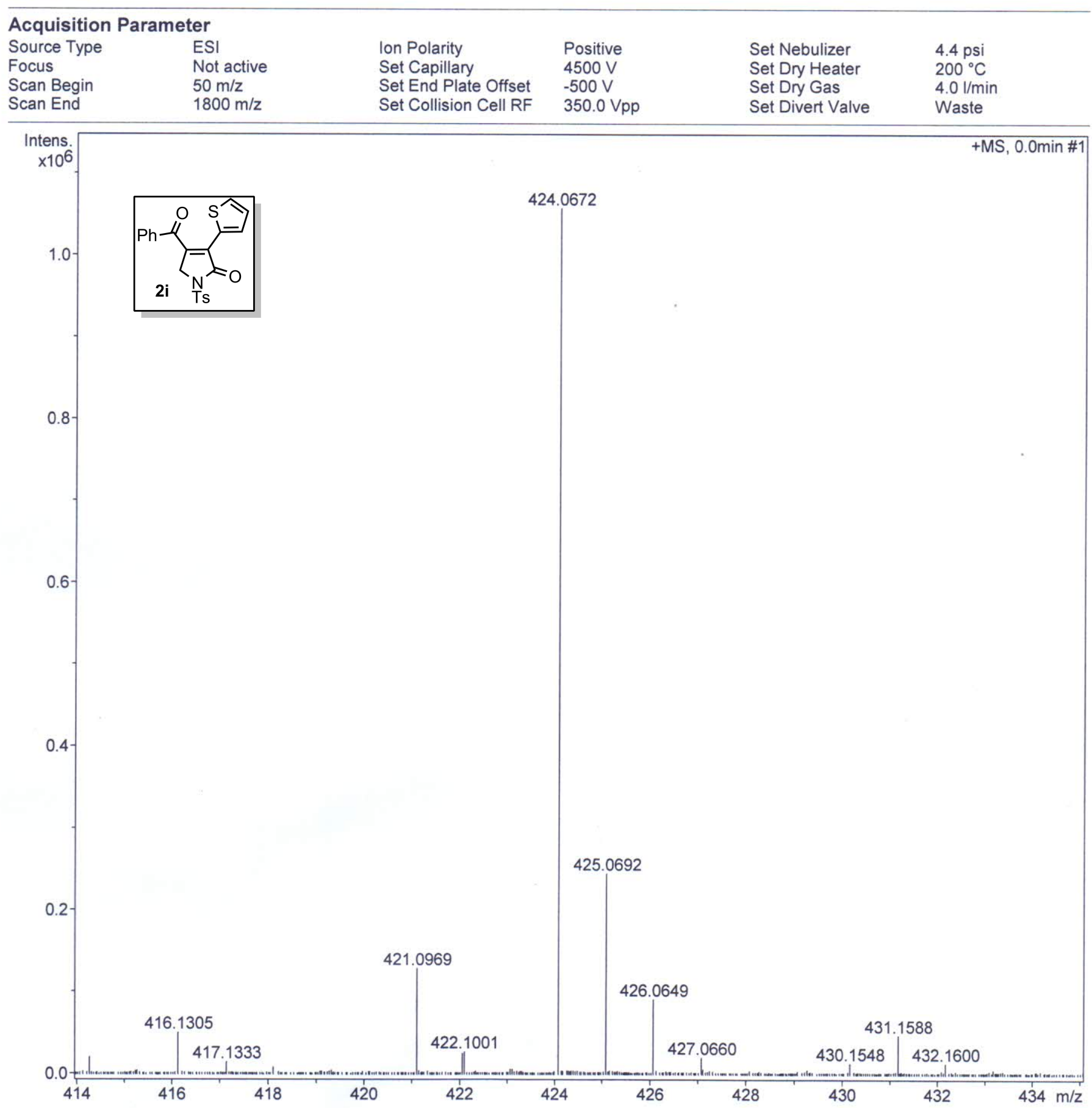




\section{BRUKER MAXIS HRMS REPORT \\ School of Chemistry \\ University of Hyderabad}

Analysis Info

Analysis Name

Method

Sample Name

Comment
D:IDatal2015IDR.AK.SAHOOINOVRP-3-127.d tune_low.m

RP-3-127
Acquisition Date

11/5/2015 2:41:54 PM

Operator

Instrument
Ramu Sridhar maXis

\begin{tabular}{lllll}
\hline \multicolumn{2}{l}{ Acquisition Parameter } & & \\
Source Type & ESI & lon Polarity & Positive & Set Nebulizer \\
Focus & Not active & Set Capillary & $4500 \mathrm{~V}$ & Set Dry Heater \\
Scan Begin & $50 \mathrm{~m} / \mathrm{z}$ & Set End Plate Offset & $-500 \mathrm{~V}$ & $200^{\circ} \mathrm{C}$ \\
Scan End & $1800 \mathrm{~m} / \mathrm{z}$ & Set Collision Cell RF & $350.0 \mathrm{Vpp}$ & Set Dry Gas \\
\hline
\end{tabular}

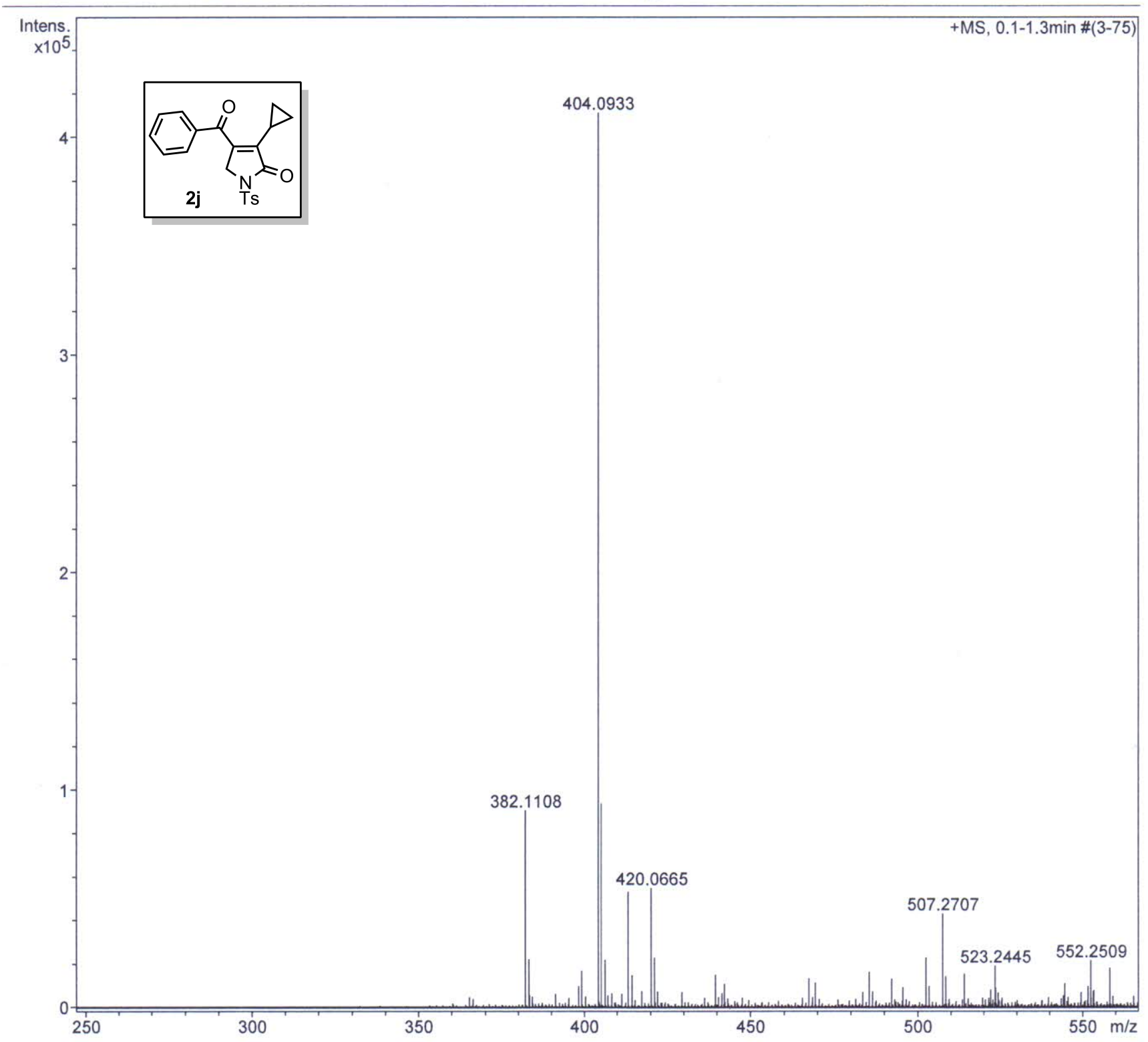




\section{BRUKER MAXIS HRMS REPORT \\ School of Chemistry \\ University of Hyderabad}

\section{Analysis Info}

Analysis Name Method

Sample Name

Comment
D:IDatal2015IDR.AK.SAHOOINOVRP-3-118.d tune_low.m

RP-3-118
Acquisition Date

Operator

Instrument
11/5/2015 2:30:14 PM

Ramu Sridhar maXis 10138

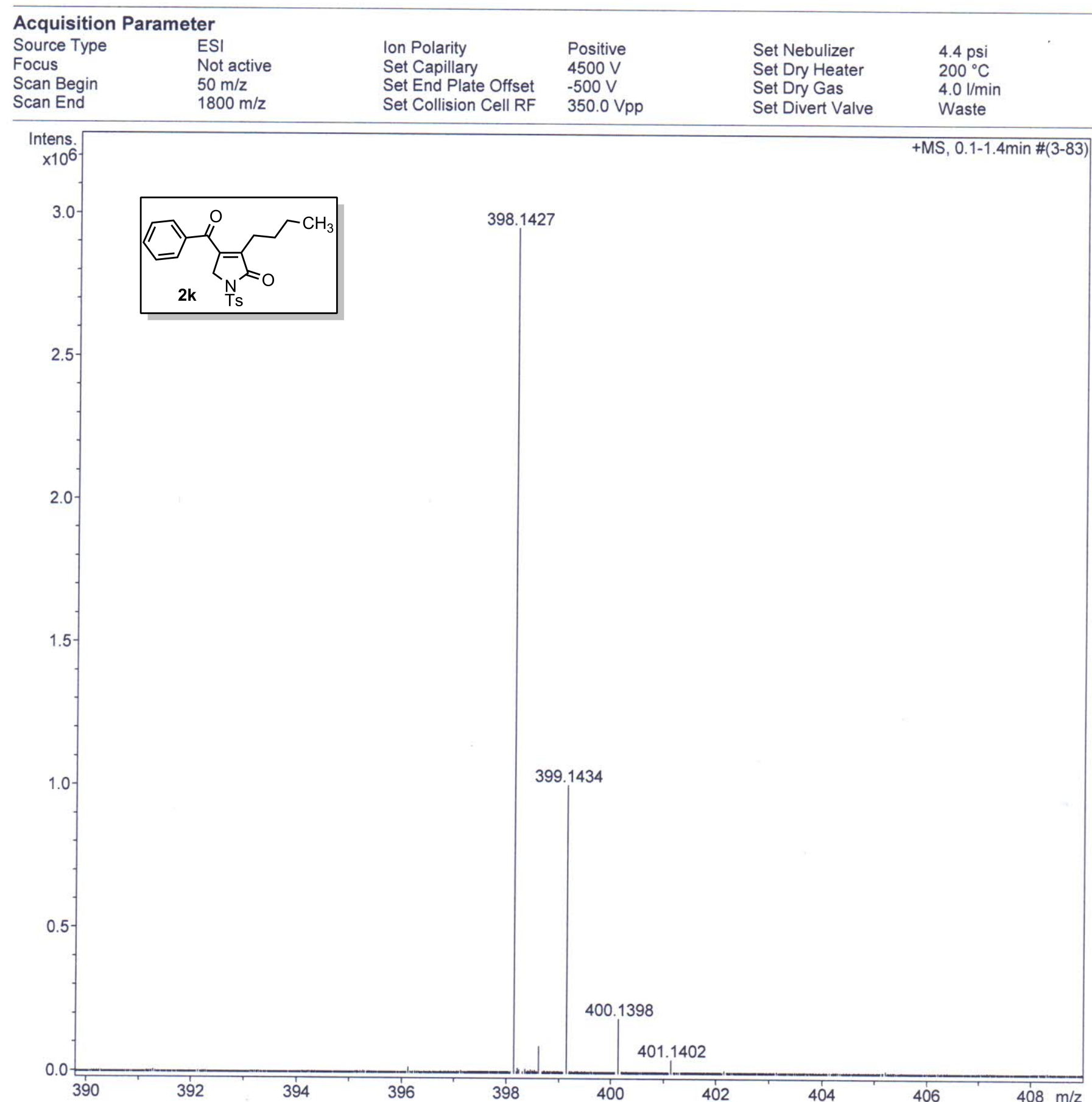




\section{UOH -SCHOOL OF CHEMISTRY -HRMS}

\section{Analysis Info}

Analysis Name

Method

Sample Name

Comment

\section{Acquisition Parameter}

Source Type

Focus

Scan Begin

Scan End

ESI

Not active

$50 \mathrm{~m} / \mathrm{z}$

$1500 \mathrm{~m} / \mathrm{z}$

Intens.

$\times 10^{6}$

3.0

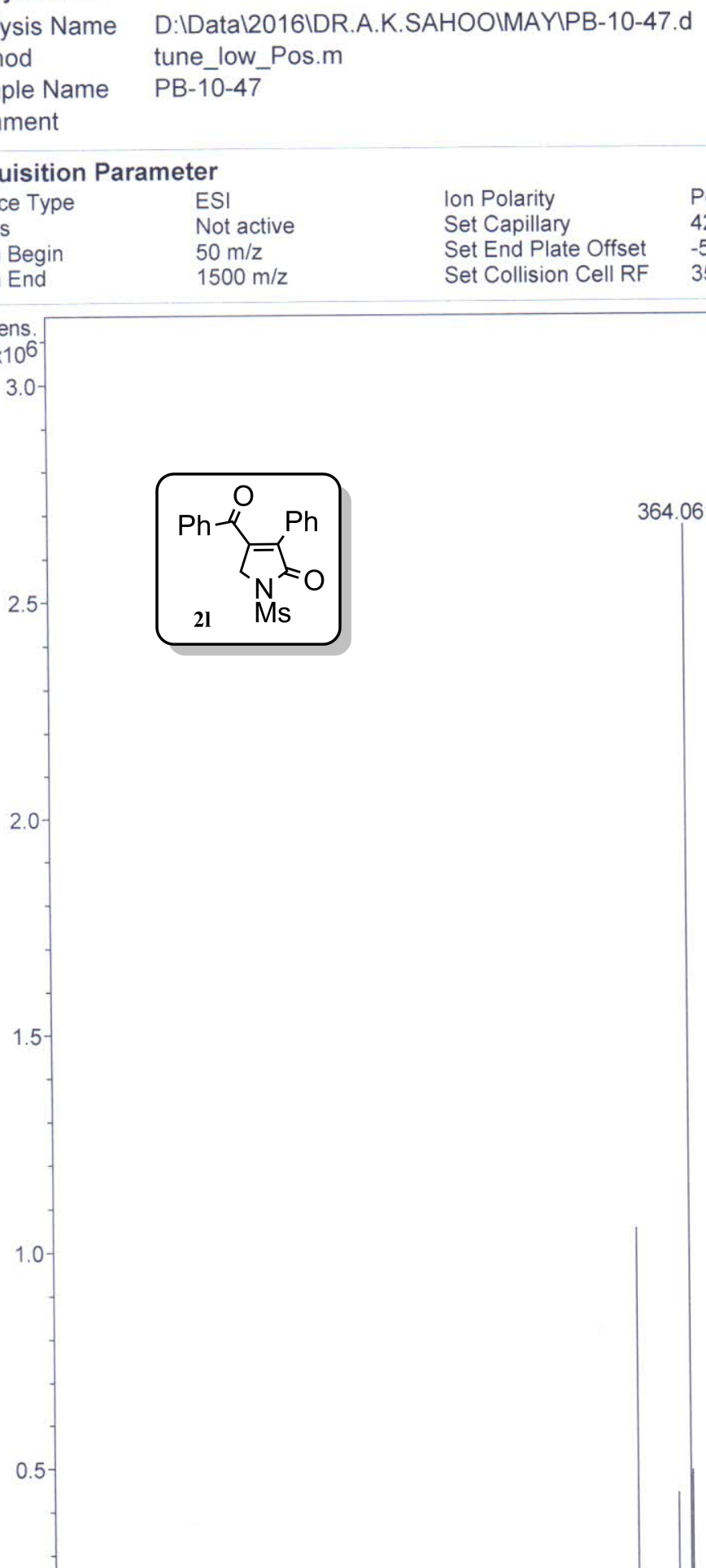

2.5. 21

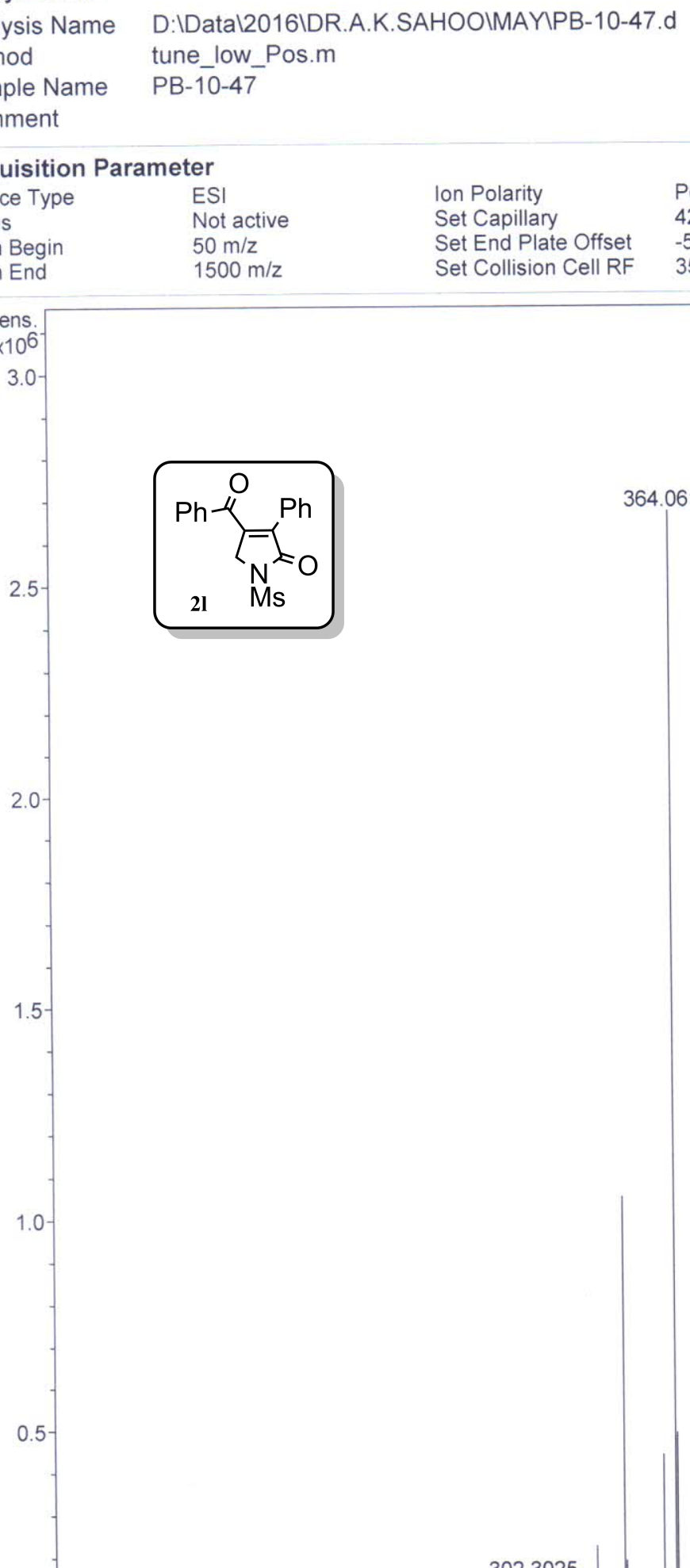

2.0

$-$

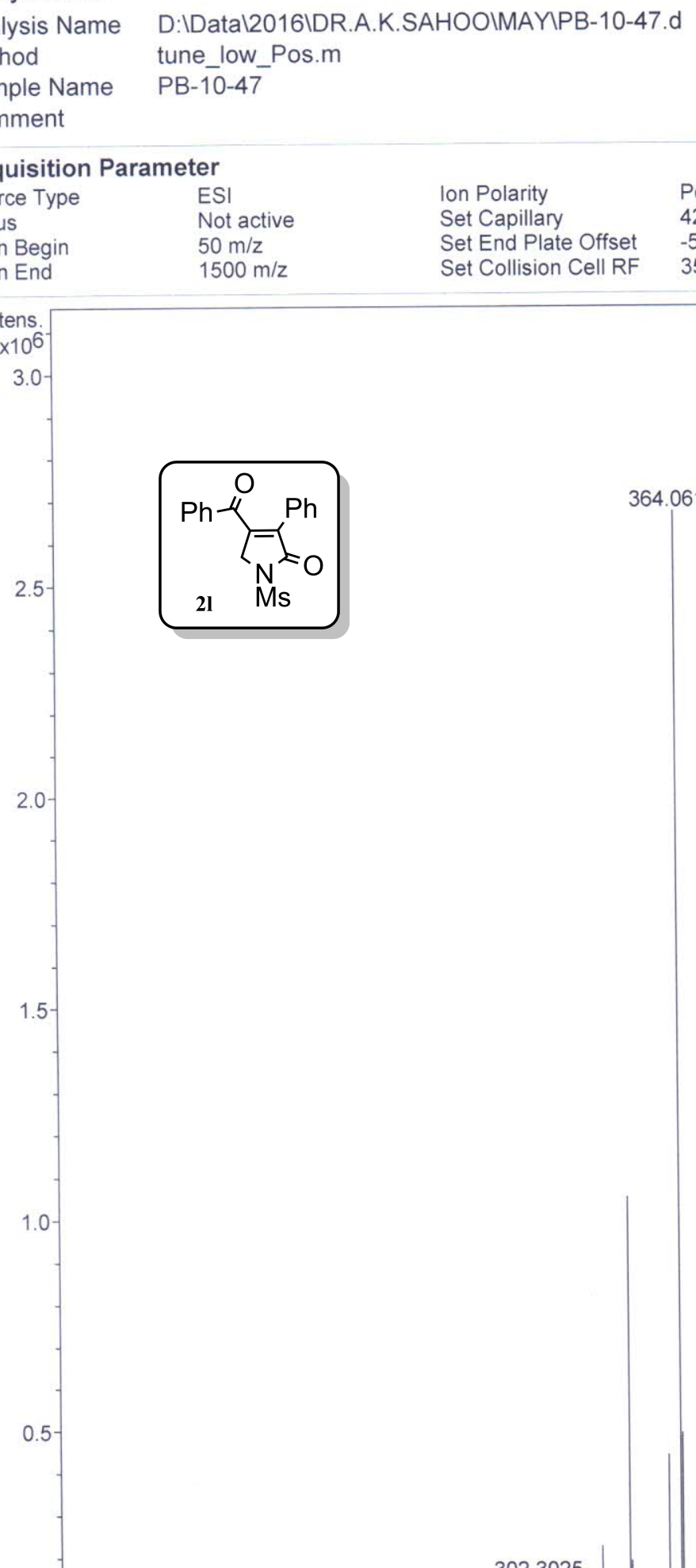

$1.5-$

(1)

1.0

$-$

$-5$

0.5

$-$

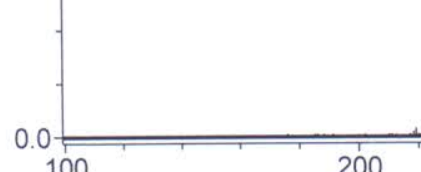

100

$\begin{array}{ll}\text { Ion Polarity } & \text { Positive } \\ \text { Set Capillary } & 4200 \mathrm{~V} \\ \text { Set End Plate Offset } & -500 \mathrm{~V} \\ \text { Set Collision Cell RF } & 350.0 \mathrm{Vpp}\end{array}$

Acquisition Date

Operator

Instrument

Rajesh Vashisth

maXis

10138

5/25/2016 4:27:27 PM

$50.0 \mathrm{Vpp}$
$0.3 \mathrm{Bar}$

$180^{\circ} \mathrm{C}$

$4.0 \mathrm{l} / \mathrm{min}$

Waste 


\section{BRUKER MAXIS HRMS REPORT \\ School of Chemistry \\ University of Hyderabad}

\section{Analysis Info}

Analysis Name Method

Sample Name

Comment
D:IDatal2014|Dr.A.K.SAHOOISEPIPB-4-118.d tune_low_Pos.m

PB-4-118-MEOH
Acquisition Date

9/5/2014 1:10:58 PM

Operator Ramu Sridhar

Instrument maXis 10138

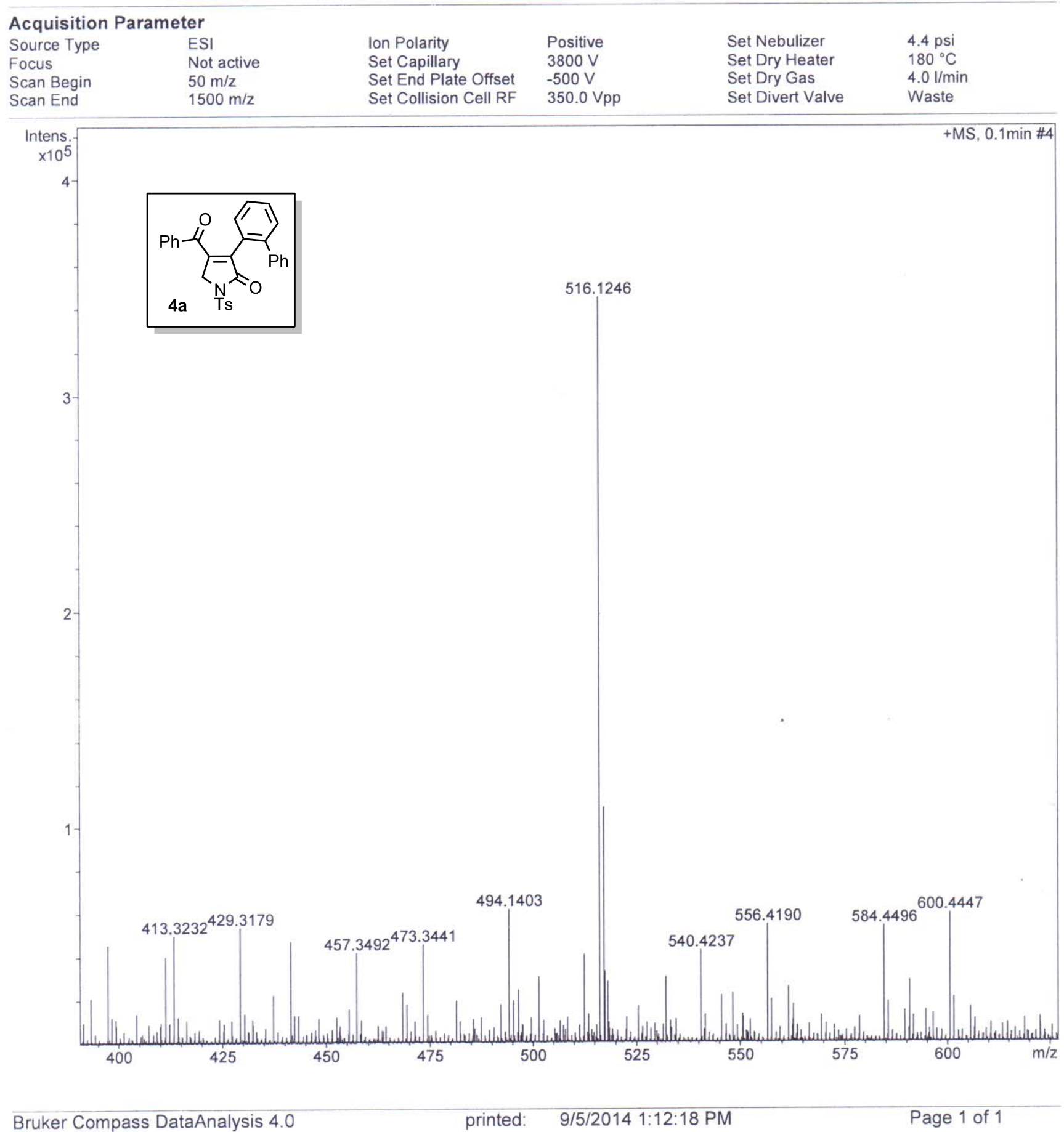




\section{BRUKER MAXIS HRMS REPORT \\ School of Chemistry \\ University of Hyderabad}

\section{Analysis Info}

Analysis Name

Method

Sample Name

Comment
D:IDatal2015IDR.AK.SAHOOIMAYYPB-5-76.d tune low.m

PB-5-76
Acquisition Date

5/13/2015 7:18:13 PM

Operator Instrument
Ramu Sridhar maXis

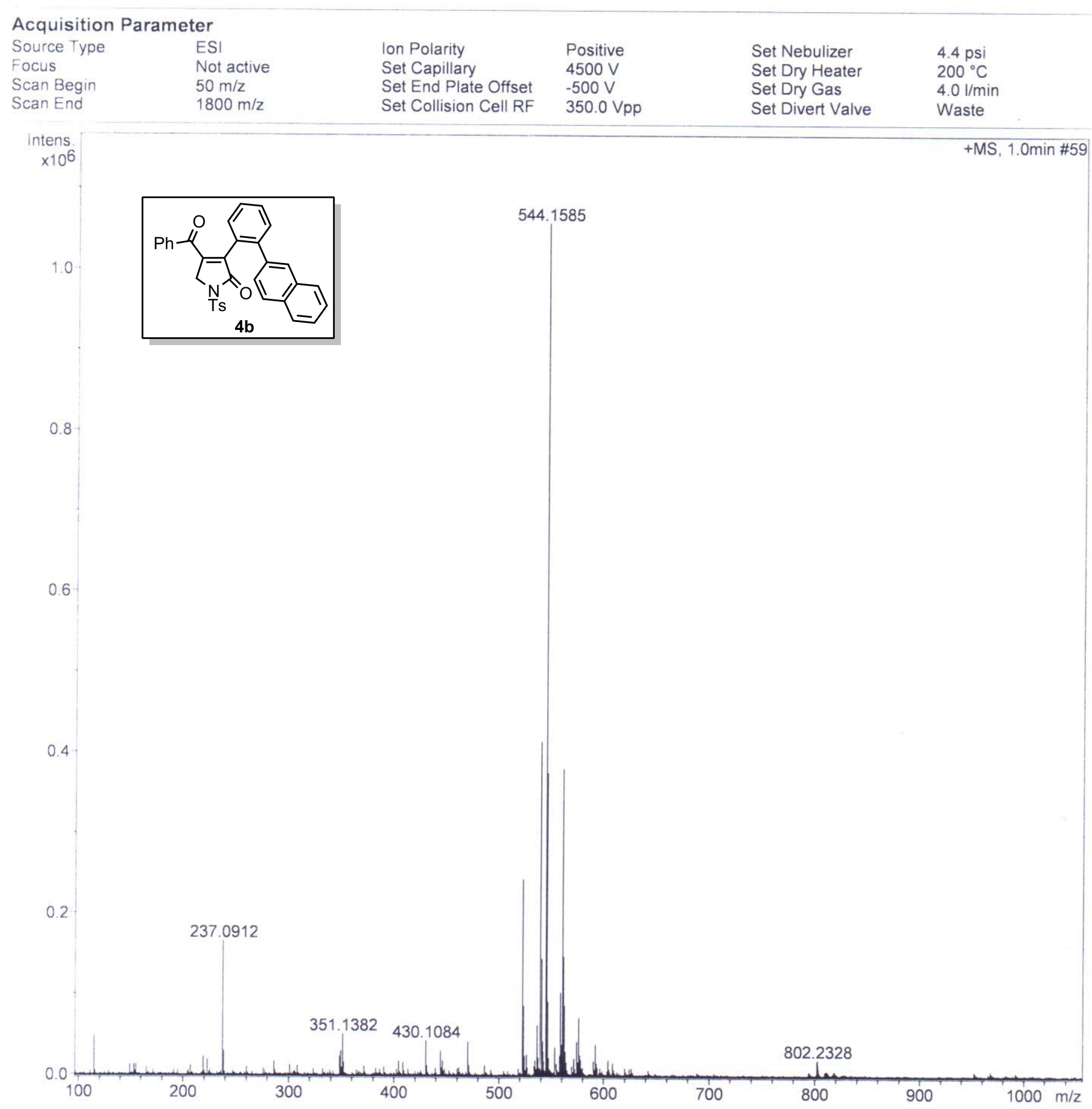




\section{BRUKER MAXIS HRMS REPORT \\ School of Chemistry \\ University of Hyderabad}

Analysis Info

Analysis Name Method

Sample Name

Comment
D:IDatal2015IDR.AK.SAHOOIMAYISn-09-119R.d tune low Pos.m

Sn-09-119-DCM-MEOH
Acquisition Date

Operator

Instrument
5/15/2015 11:28:12 AM

Ramu Sridhar maXis

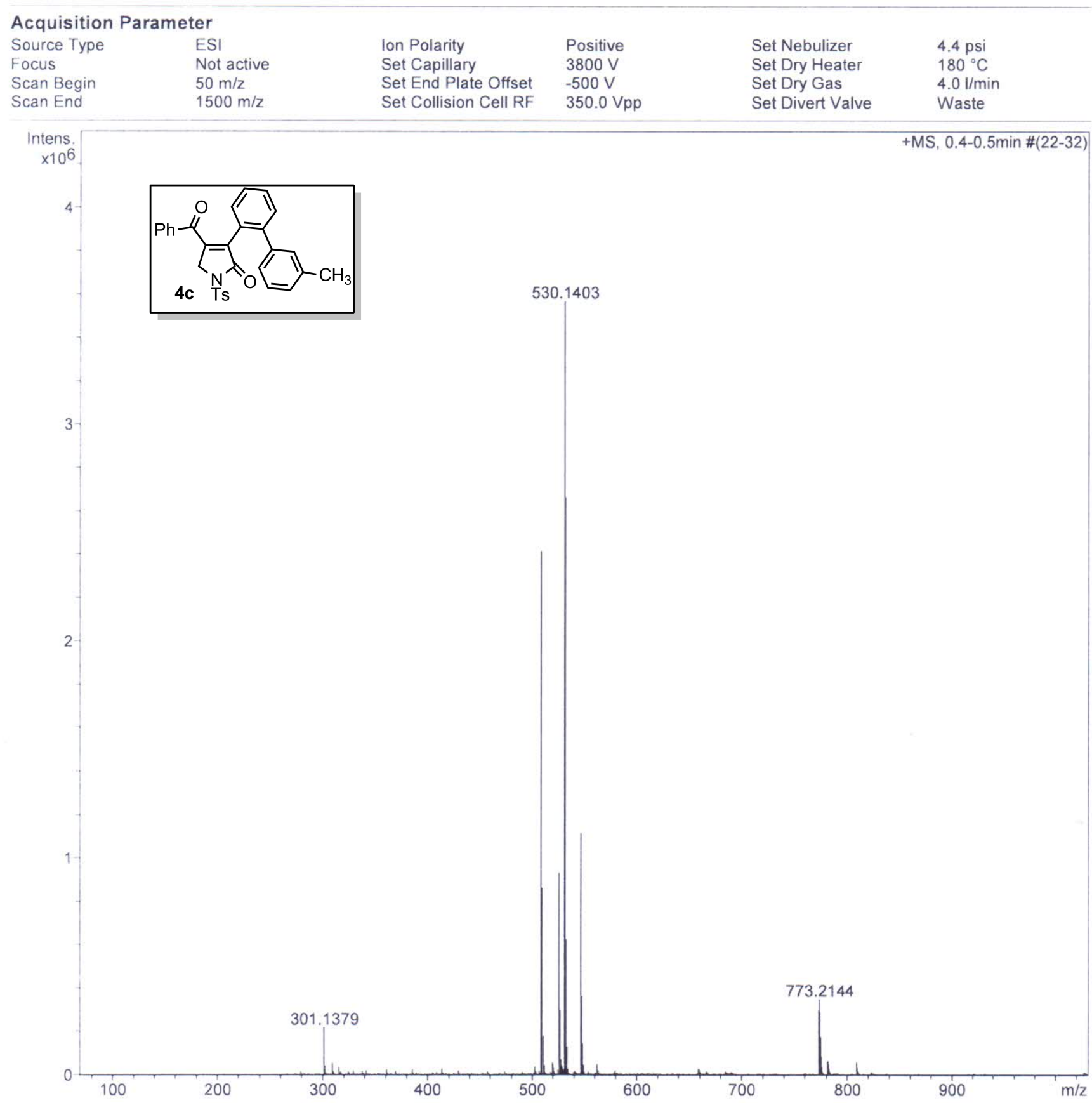




\section{BRUKER MAXIS HRMS REPORT \\ School of Chemistry \\ University of Hyderabad}

Analysis Info

Analysis Name

Method

Sample Name

Comment
D:IDatal2015IDR.AK.SAHOOIMAYMB-5-77.d tune_low.m PB-5-77
Acquisition Date

5/12/2015 6:55:48 PM

Operator Ramu Sridhar

Instrument maXis

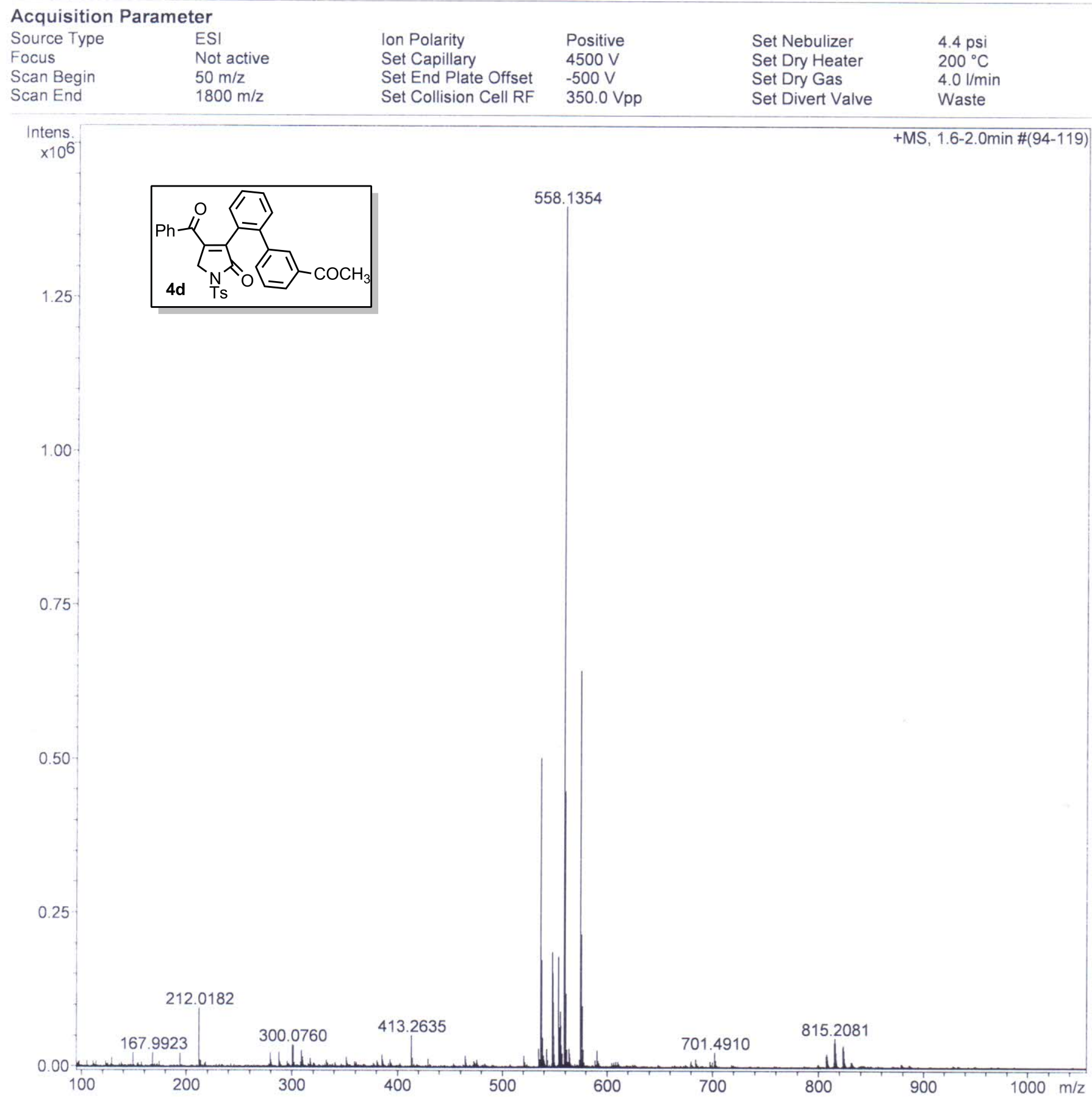




\section{BRUKER MAXIS HRMS REPORT \\ School of Chemistry University of Hyderabad}

Analysis Info

Analysis Name

Method

Sample Name

Comment
Acquisition Date

5/13/2015 6:12:45 PM

D:IDatal2015IDR.AK.SAHOOIMAYIPB-A-206.d tune low.m

PB-A-206
Ramu Sridhar

maXis
Instrument

\section{Acquisition Parameter}

Source Type

Focus

ESI

Scan Begin

Not active

Scan End

$50 \mathrm{~m} / \mathrm{z}$ $1800 \mathrm{~m} / \mathrm{z}$

Ion Polarity

Set Capillary

Set End Plate Offset

Positive

Set Collision Cell RF

$350.0 \mathrm{Vpp}$

$4.4 \mathrm{psi}$

Set Nebulizer

Set Dry Heater

Set Dry Gas

Set Divert Valve

$4.0 \mathrm{l} / \mathrm{min}$

Waste

Intens.
$\times 10^{5}$

+MS, 0.1-2.0min \#(7-118)

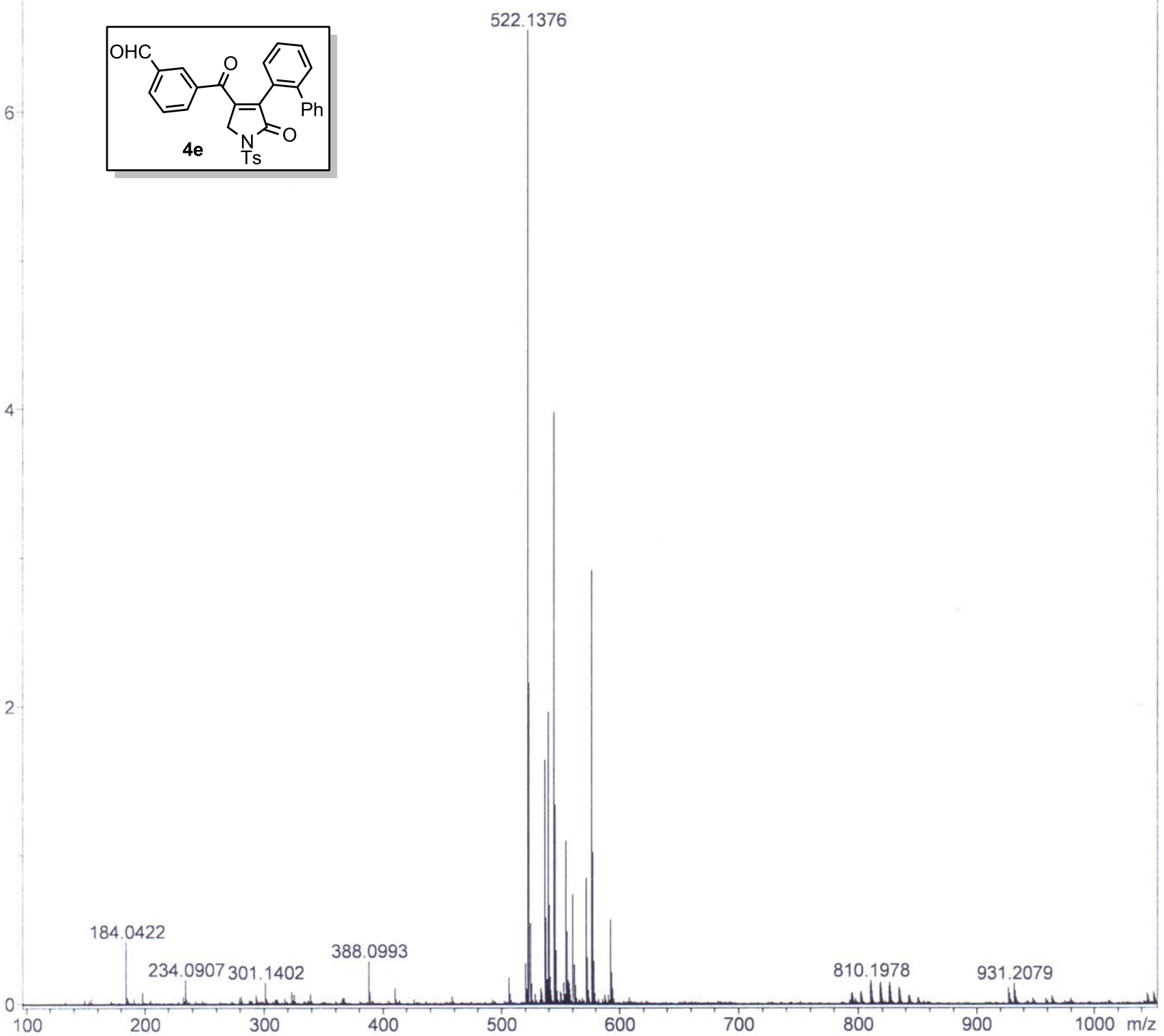




\section{UOH -SCHOOL OF CHEMISTRY -HRMS}

\section{Analysis Info}

Analysis Name Method

Sample Name

Comment

\section{Acquisition Parameter}

Source Type ESI

Focus Not active

Scan Begin $\quad 50 \mathrm{~m} / \mathrm{z}$

Scan End $\quad 800 \mathrm{~m} / \mathrm{z}$
2016IDR.A.K.SAHOOIMARCHIPB-4-153R1.d TL-P.m

B-4-153-MEOH
Acquisition Date $\quad 3 / 22 / 2016$ 11:22:10 AM

Operator

Instrument

Rajesh Vashisth maXis 10138

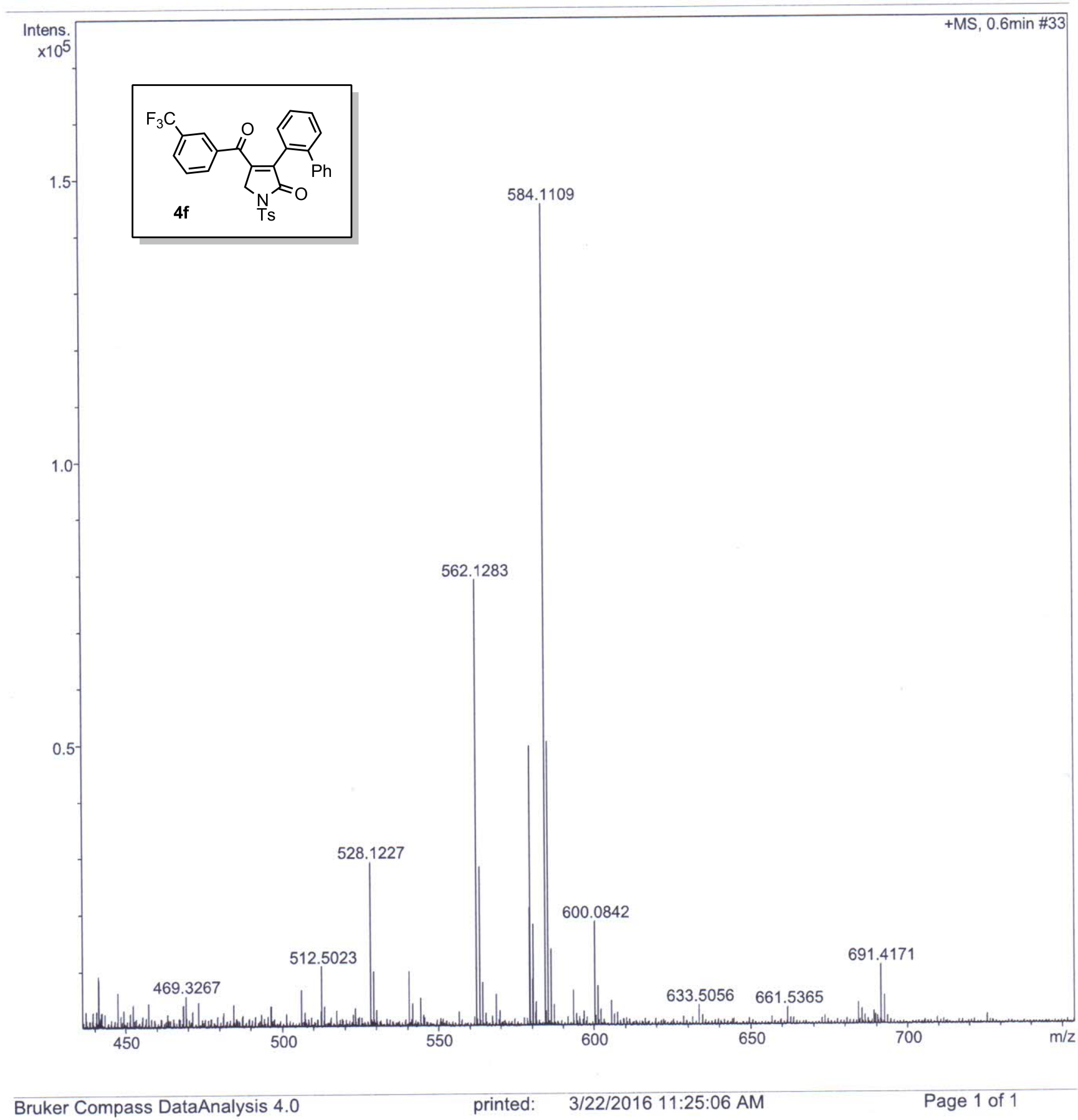




\section{BRUKER MAXIS HRMS REPORT \\ School of Chemistry \\ University of Hyderabad}

Analysis Info

Analysis Name

Method

Sample Name

Comment
D:IDatal2015IDR.AK.SAHOOIMAYPB-A-178.d

tune_low_Pos-R2.m

PB-A-178

4
Acquisition Date

5/14/2015 5:31:17 PM

Operator

Instrument
Ramu Sridhar maXis

\section{Acquisition Parameter}

Source Type ESI

Focus

Scan Begin

Scan End

Not active

$50 \mathrm{~m} / \mathrm{z}$

$2580 \mathrm{~m} / \mathrm{z}$
Ion Polarity

Set Capillary

Set End Plate Offset

Set Collision Cell RF
Positive

$3800 \mathrm{~V}$

$-500 \mathrm{~V}$

$350.0 \mathrm{Vpp}$
Set Nebulizer

Set Dry Heater

Set Dry Gas

Set Divert Valve
$4.4 \mathrm{psi}$

$180^{\circ} \mathrm{C}$

$4.0 \mathrm{l} / \mathrm{min}$

Waste

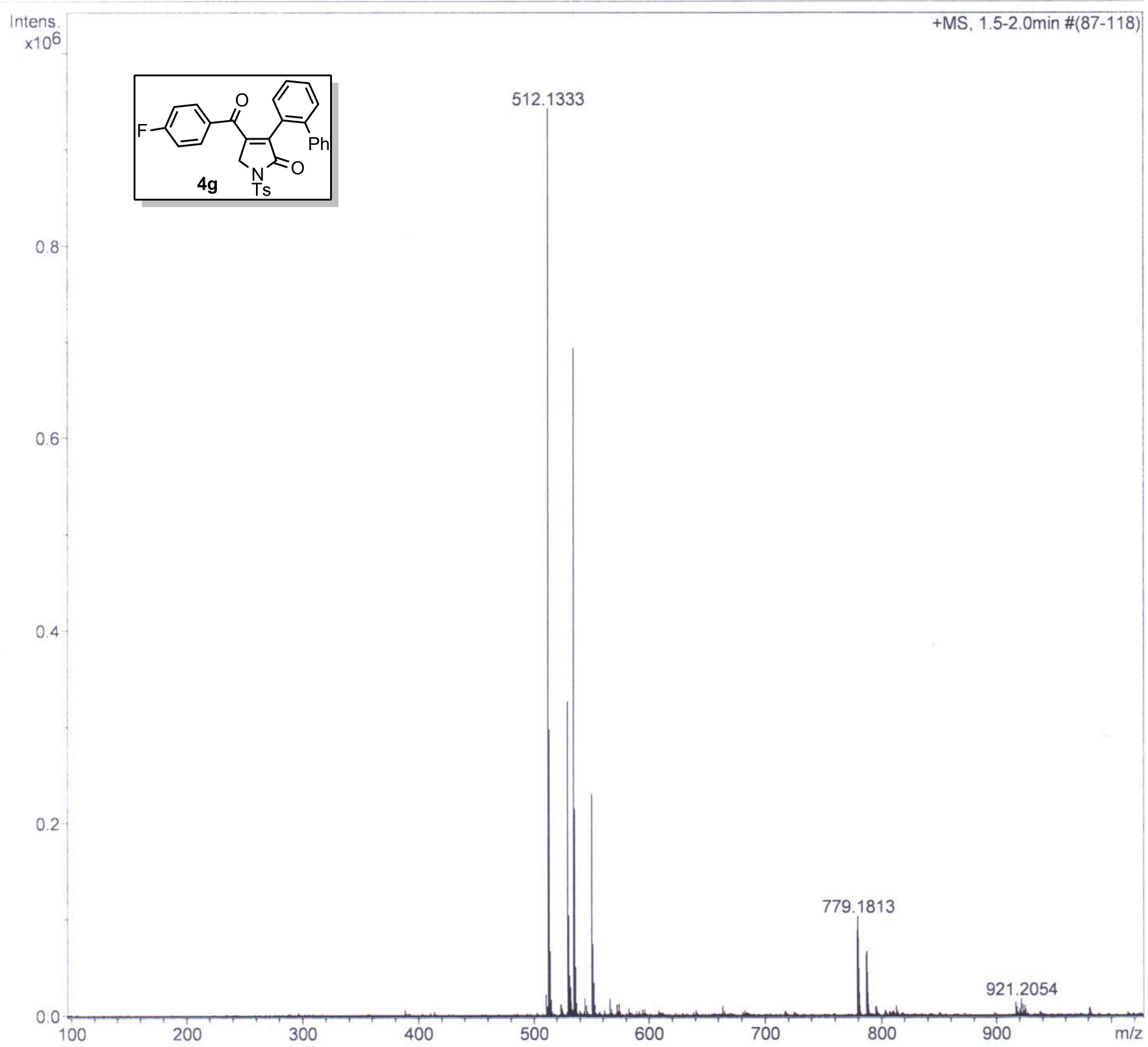




\section{BRUKER MAXIS HRMS REPORT \\ School of Chemistry \\ University of Hyderabad}

Analysis Info

Analysis Name

Method

Sample Name

Comment
D:IDatal2015IDR.AK.SAHOOIMAYIPB-5-48.d tune_low.m

PB-5-48
Acquisition Date

Operator

Instrument
5/12/2015 3:47:30 PM

Ramu Sridhar

maXis

10138

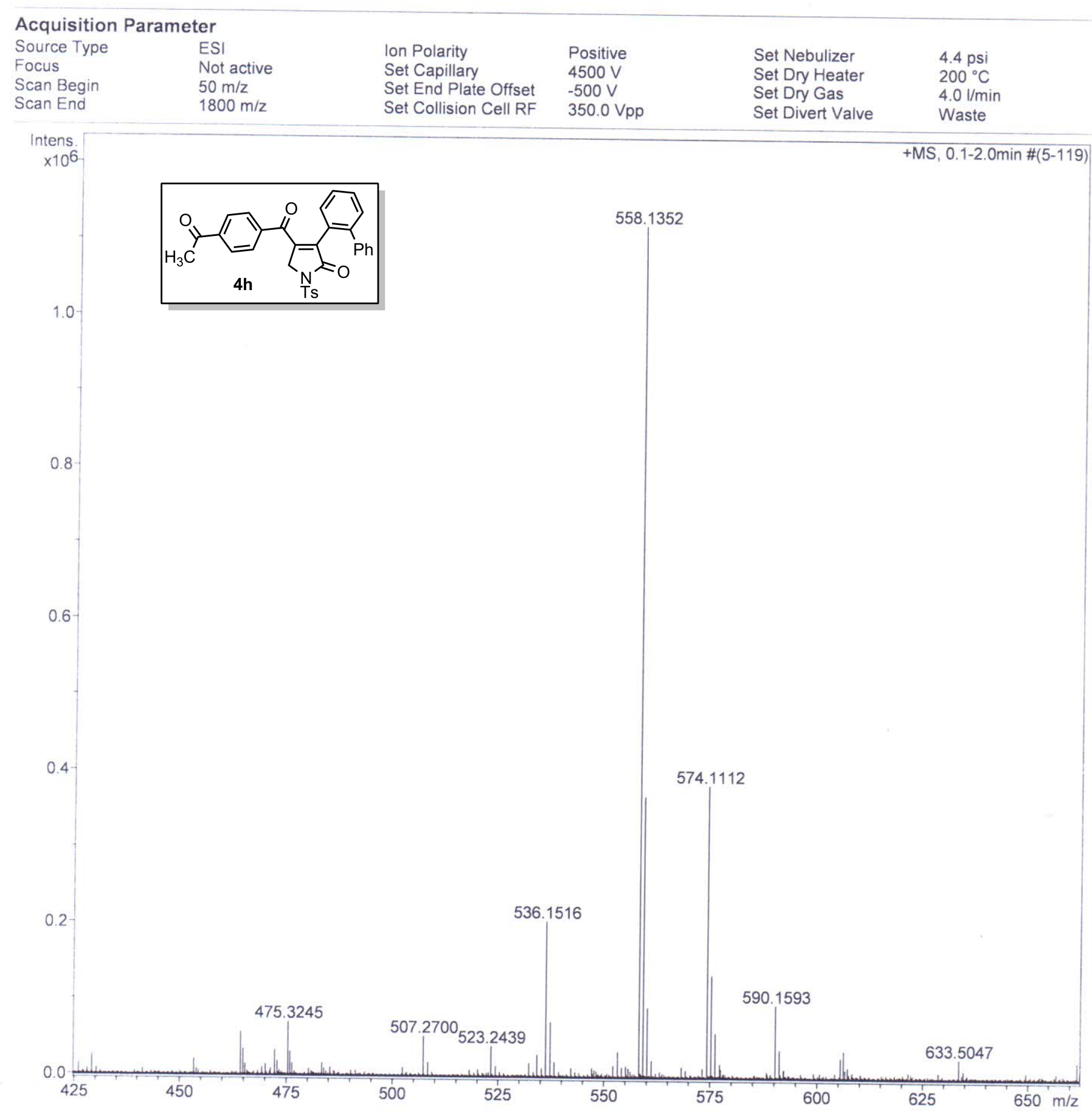




\section{BRUKER MAXIS HRMS REPORT \\ School of Chemistry \\ University of Hyderabad}

\section{Analysis Info}

Analysis Name Method

Sample Name

Comment
Acquisition Date 5/14/2015 5:22:52 PM

D:IDatal2015IDR.AK.SAHOOIMAYIPB-A-186.d tune low_Pos-R2.m

PB-A-186
Operator

Instrument
Ramu Sridhar

maXis

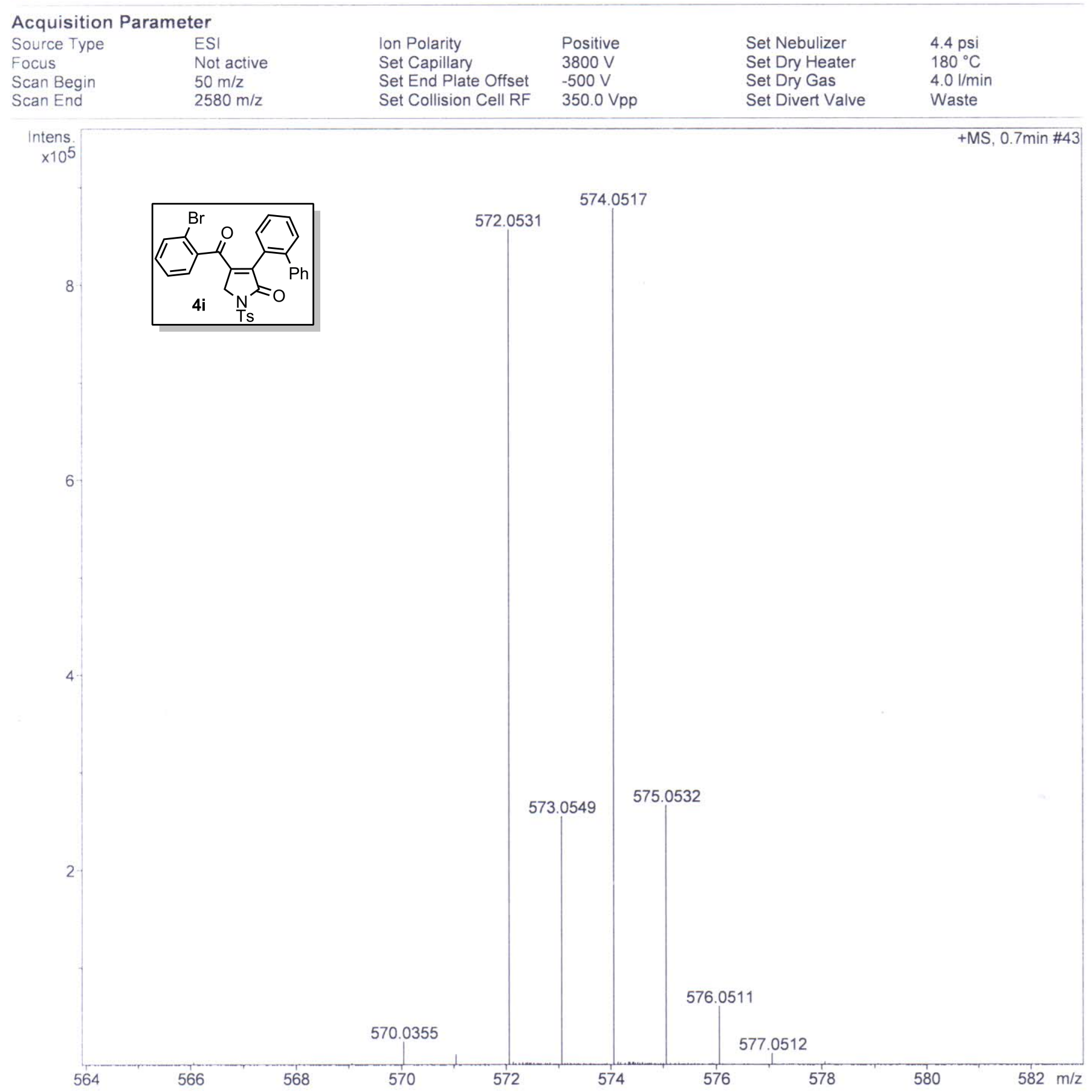




\section{BRUKER MAXIS HRMS REPORT \\ School of Chemistry \\ University of Hyderabad}

Analysis Info

Analysis Name

Method

Sample Name

Comment

Acquisition Parameter

Source Type ESI

Focus

Scan Begin

Not active

$50 \mathrm{~m} / \mathrm{z}$

Scan End

$1700 \mathrm{~m} / \mathrm{z}$

D:IDatal2015IDR.AK.SAHOOIOctIPB-4-157.d

tune_low.m

PB-4-157-MEOH

Acquisition Date

10/21/2015 4:37:15 PM

Operator

Instrument

Ramu Sridhar

maXis

10138

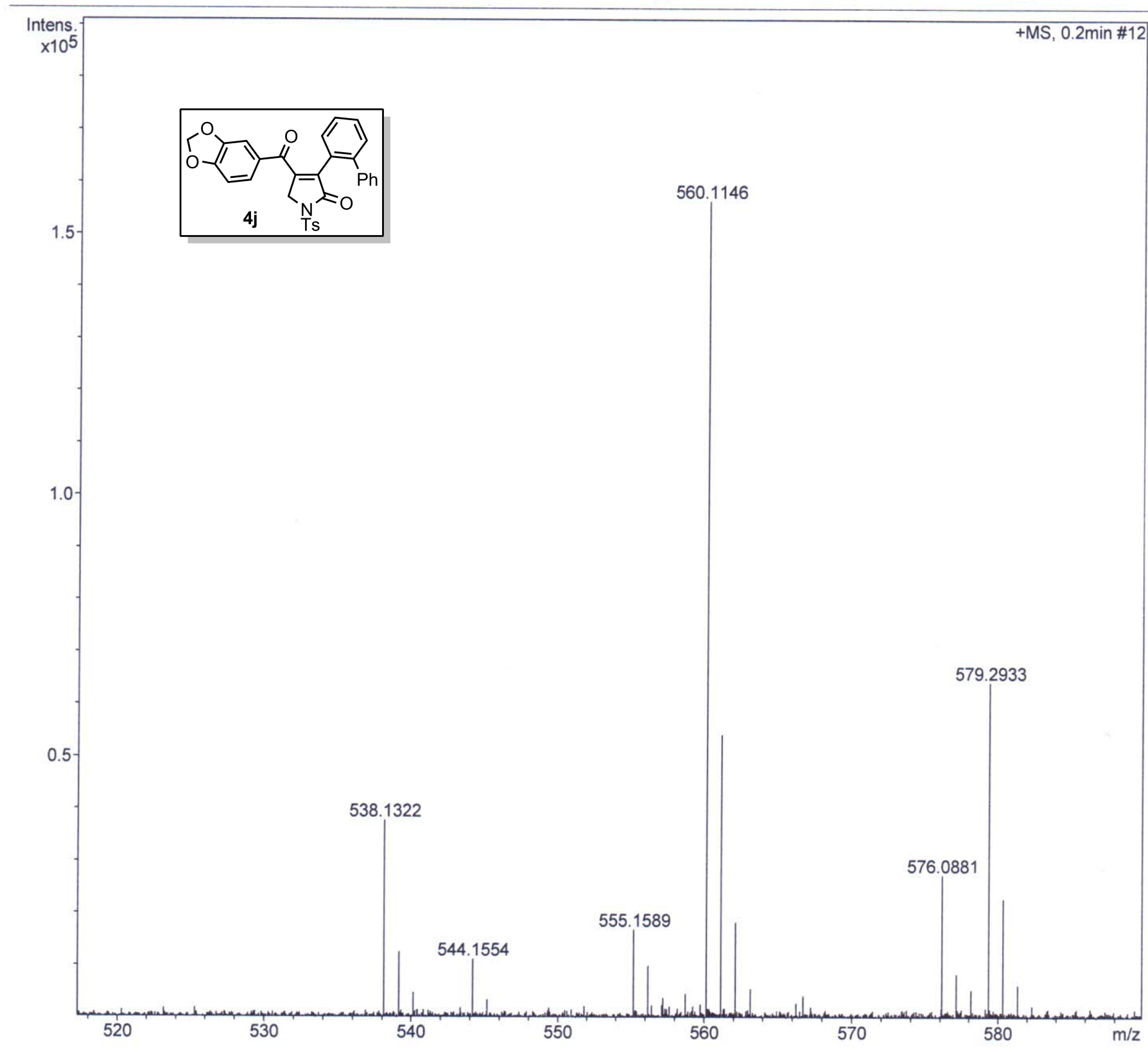




\section{BRUKER MAXIS HRMS REPORT \\ School of Chemistry \\ University of Hyderabad}

Analysis Info

Analysis Name

Method

Sample Name

Comment
D:IDatal2015IDR.AK.SAHOOlaprIIPB-4-180.d

tune_low.m

PB-4-180-DCM-MEOH
Acquisition Date

Operator

Instrument
4/27/2015 12:54:21 PM

Ramu Sridhar

maXis

Acquisition Parameter

Source Type

Focus Not active

Scan Begin $\quad 50 \mathrm{~m} / \mathrm{z}$

Scan End $\quad 1800 \mathrm{~m} / \mathrm{z}$

$\times 10^{5}$

Ion Polarity

Set Capillary

Set End Plate Offset

Set Collision Cell RF

Positive
$4500 \mathrm{~V}$
$-500 \mathrm{~V}$
$350.0 \mathrm{Vpp}$

Set Nebulizer

Set Dry Heater

Set Dry Gas

Set Divert Valve
$4.4 \mathrm{psi}$

$200^{\circ} \mathrm{C}$

$4.0 \mathrm{l} / \mathrm{min}$ Waste

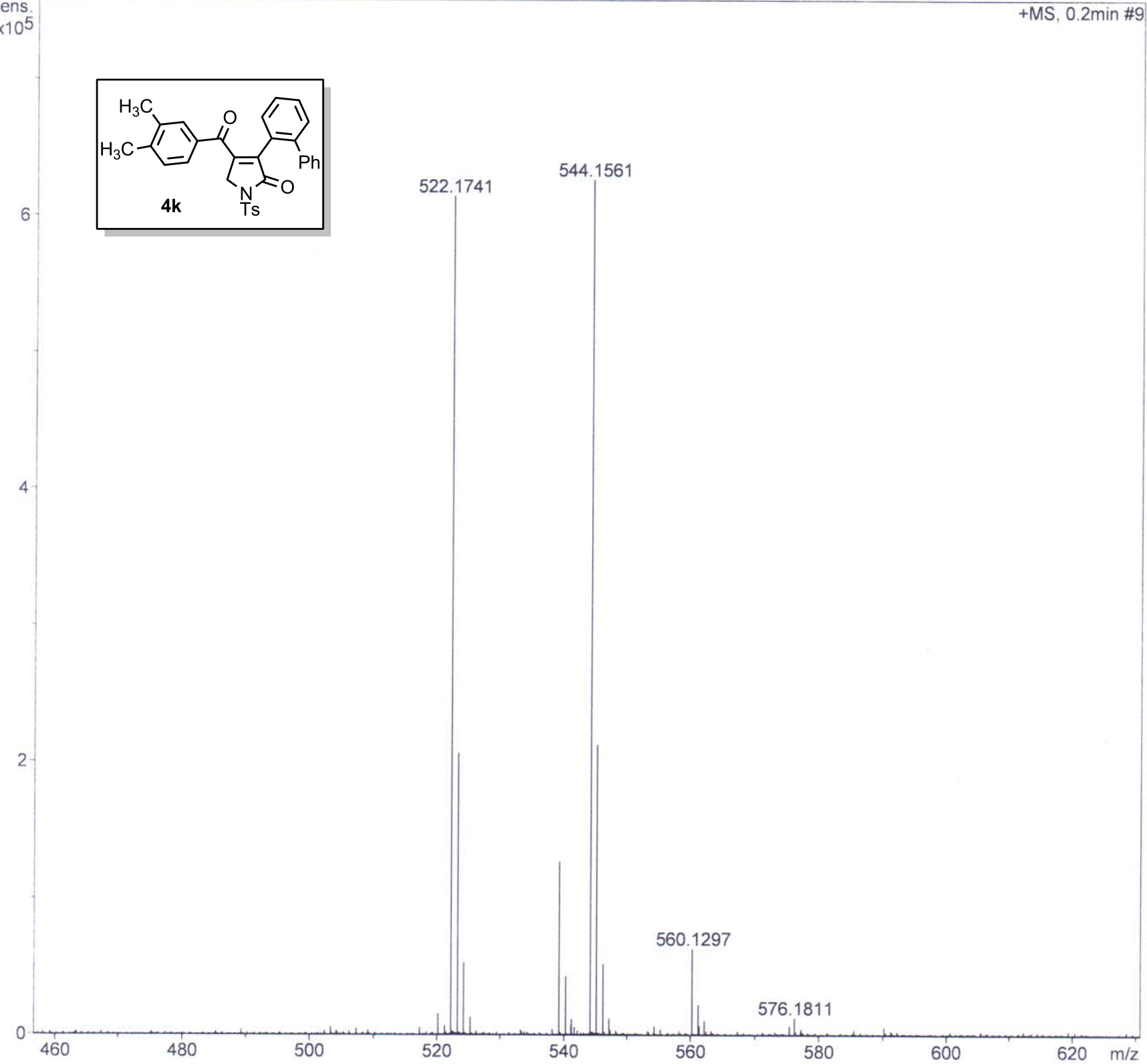




\section{BRUKER MAXIS HRMS REPORT \\ School of Chemistry \\ University of Hyderabad}

Analysis Info

Analysis Name

Method

Sample Name

Comment
Acquisition Date

D:IDatal2015IDR.AK.SAHOOIFEBISn-09-131.d tune low Pos.m

Sn-09-131
Operator

Instrument
2/23/2015 8:48:14 PM

Ramu Sridhar maXis 10138

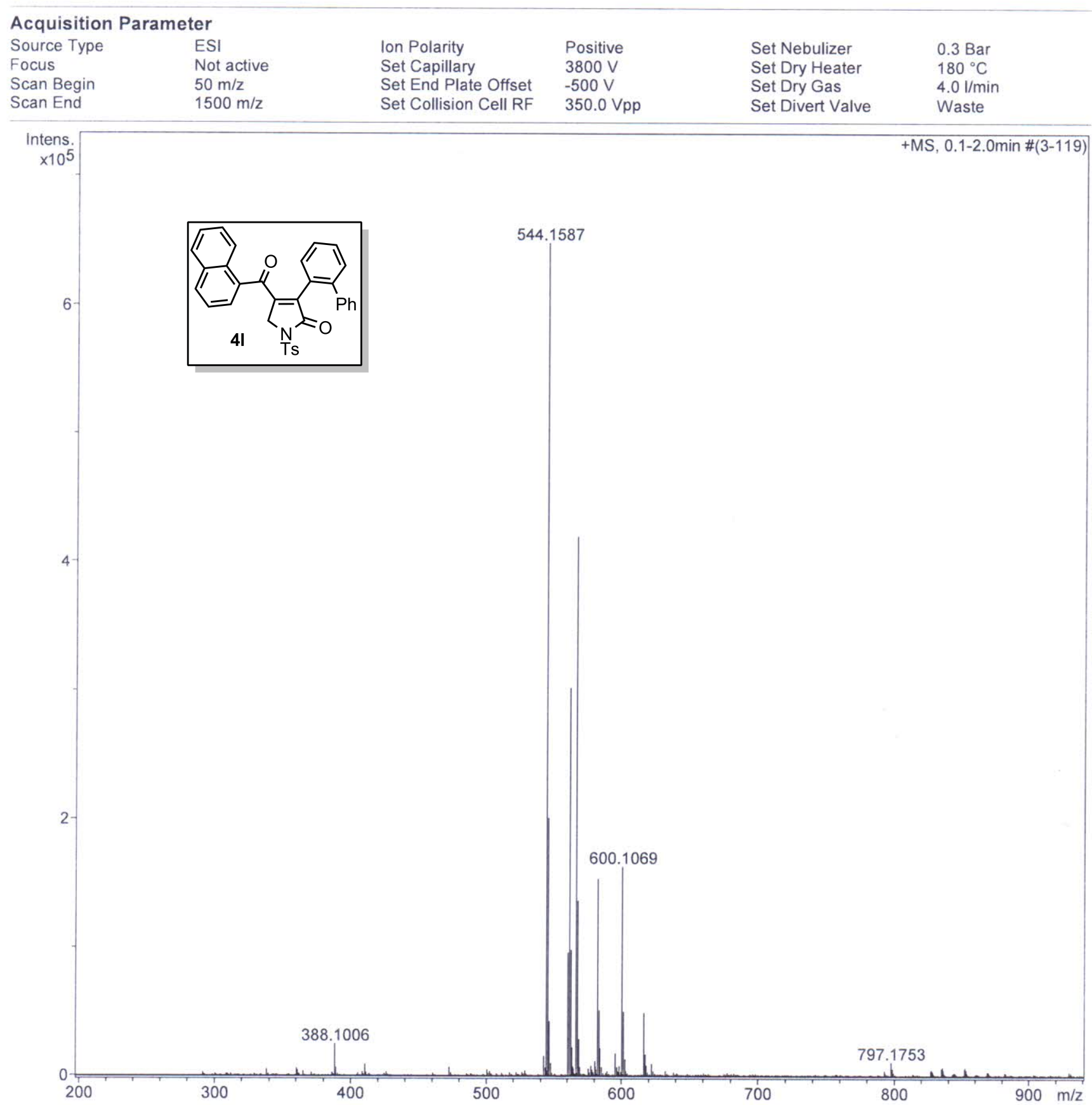




\section{BRUKER MAXIS HRMS REPORT \\ School of Chemistry University of Hyderabad}

Analysis Info

Analysis Name

Method

Sample Name

Comment
Acquisition Date

D:IDatal2015IDR.AK.SAHOOIFEBISn-09-132.d

tune_low_Pos-R2.m

Sn-09-132

Operator

Instrument
2/23/2015 8:20:30 PM

Ramu Sridhar maXis

\begin{tabular}{|c|c|c|c|c|c|}
\hline \multicolumn{6}{|c|}{ Acquisition Parameter } \\
\hline Source Type & ESI & Ion Polarity & Positive & Set Nebulizer & $0.3 \mathrm{Bar}$ \\
\hline Focus & Not active & Set Capillary & $3800 \mathrm{~V}$ & Set Dry Heater & $180^{\circ} \mathrm{C}$ \\
\hline Scan Begin & $50 \mathrm{~m} / \mathrm{z}$ & Set End Plate Offset & $-500 \mathrm{~V}$ & Set Dry Gas & $4.0 \mathrm{l} / \mathrm{min}$ \\
\hline Scan End & $2580 \mathrm{~m} / \mathrm{z}$ & Set Collision Cell RF & $350.0 \mathrm{Vpp}$ & Set Divert Valve & Waste \\
\hline
\end{tabular}

Set End Plate Offset
Set Collision Cell RF

$350.0 \mathrm{Vpp}$

+MS, 1.4-1.9min \#(82-110)

Intens.

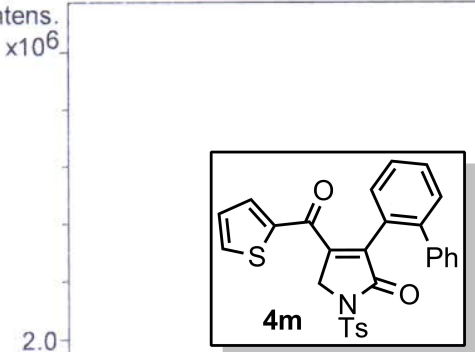

2.0

$4 \mathrm{~m} \quad \mathrm{~T}$

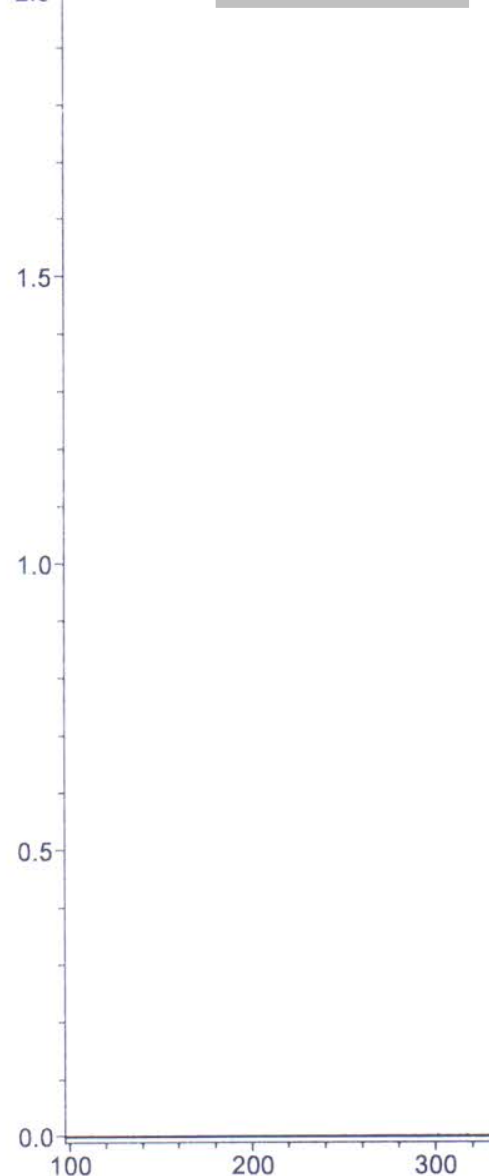




\section{BRUKER MAXIS HRMS REPORT \\ School of Chemistry \\ University of Hyderabad}

Analysis Info

Analysis Name

Method

Sample Name

Comment
Acquisition Date

5/13/2015 6:41:27 PM

D:IDatal2015IDR.AK.SAHOOIMAYPB-5-49.d tune_low_Pos-R2.m

PB-5-49
Operator

Instrument
Ramu Sridhar maXis

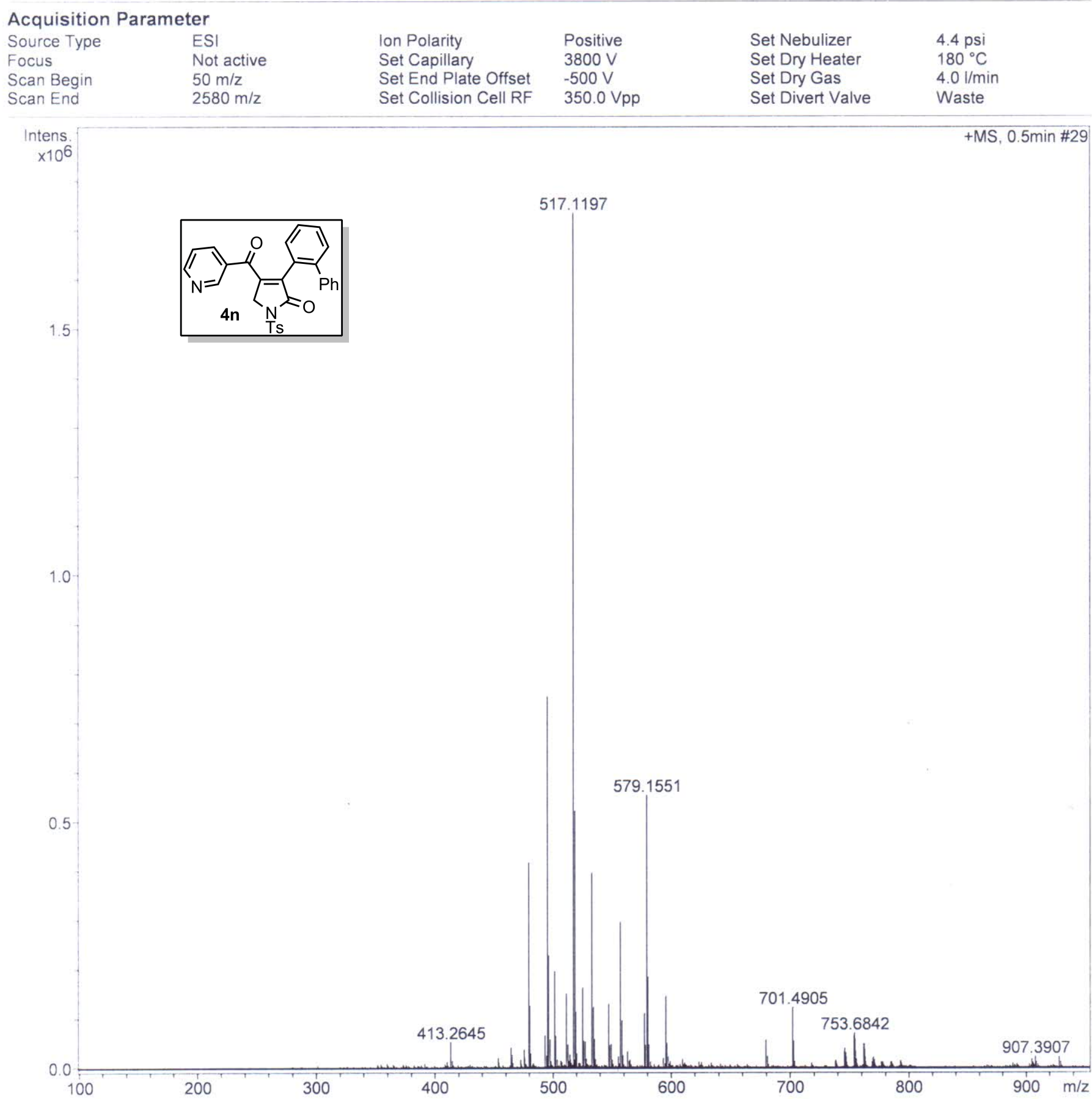




\section{BRUKER MAXIS HRMS REPORT \\ School of Chemistry University of Hyderabad}

Analysis Info

Analysis Name

Method

Sample Name

Comment
D:IDatal2015IDR.AK.SAHOOIOctIPB-8-77R.d tune_low_Pos-R2.m

PB-8-77-MEOH
Acquisition Date

10/19/2015 4:05:26 PM

Operator Ramu Sridhar

Instrument maXis

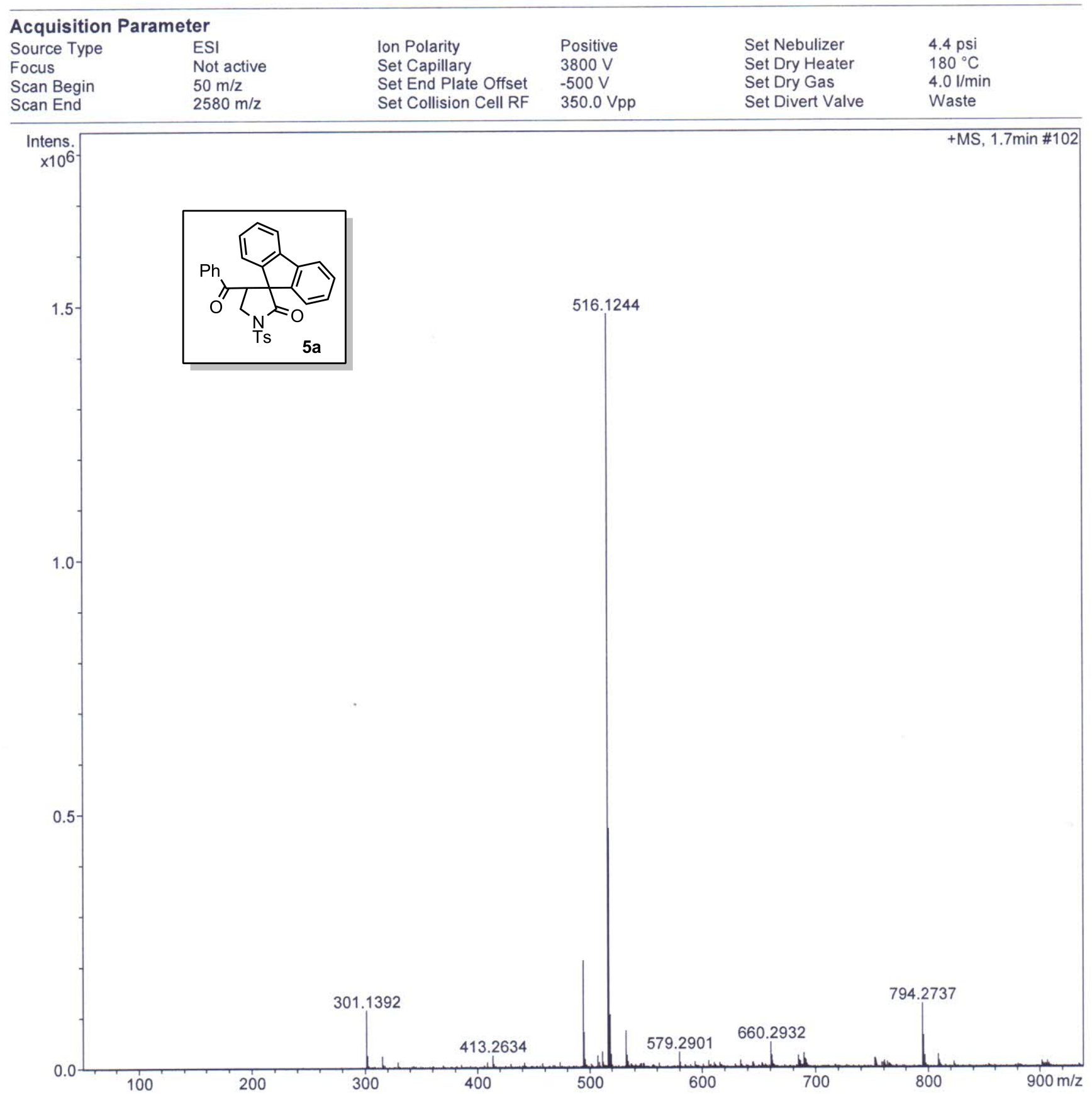




\section{BRUKER MAXIS HRMS REPORT \\ School of Chemistry \\ University of Hyderabad}

Analysis Info

Analysis Name

Method

Sample Name

D:IDatal2015IDR.AK.SAHOOINOMPB-8-133.d

tune_low.m

PB-8-133

Comment
Acquisition Date

Operator

Instrument
11/30/2015 4:39:03 PM

Ramu Sridhar

maXis

10138

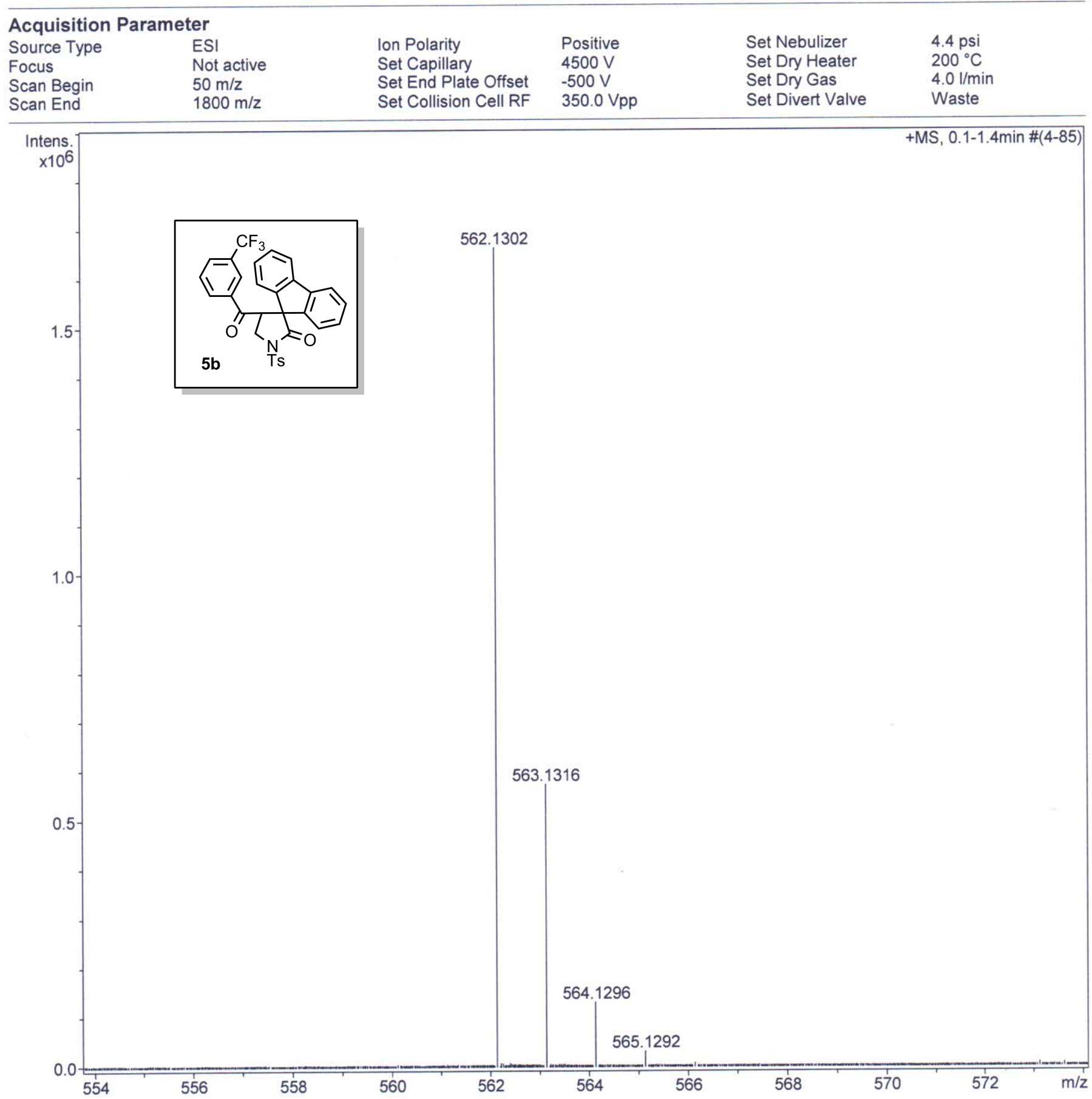




\section{BRUKER MAXIS HRMS REPORT \\ School of Chemistry \\ University of Hyderabad}

Analysis Info

Analysis Name Method

Sample Name

Comment
Acquisition Date

D:IDatal2015IDR.AK.SAHOOIOct11PB-8-77R1.d tune_low_Pos.m PB-8-77-MEOH
10/28/2015 1:11:02 PM

Operator Ramu Sridhar

Instrument maXis

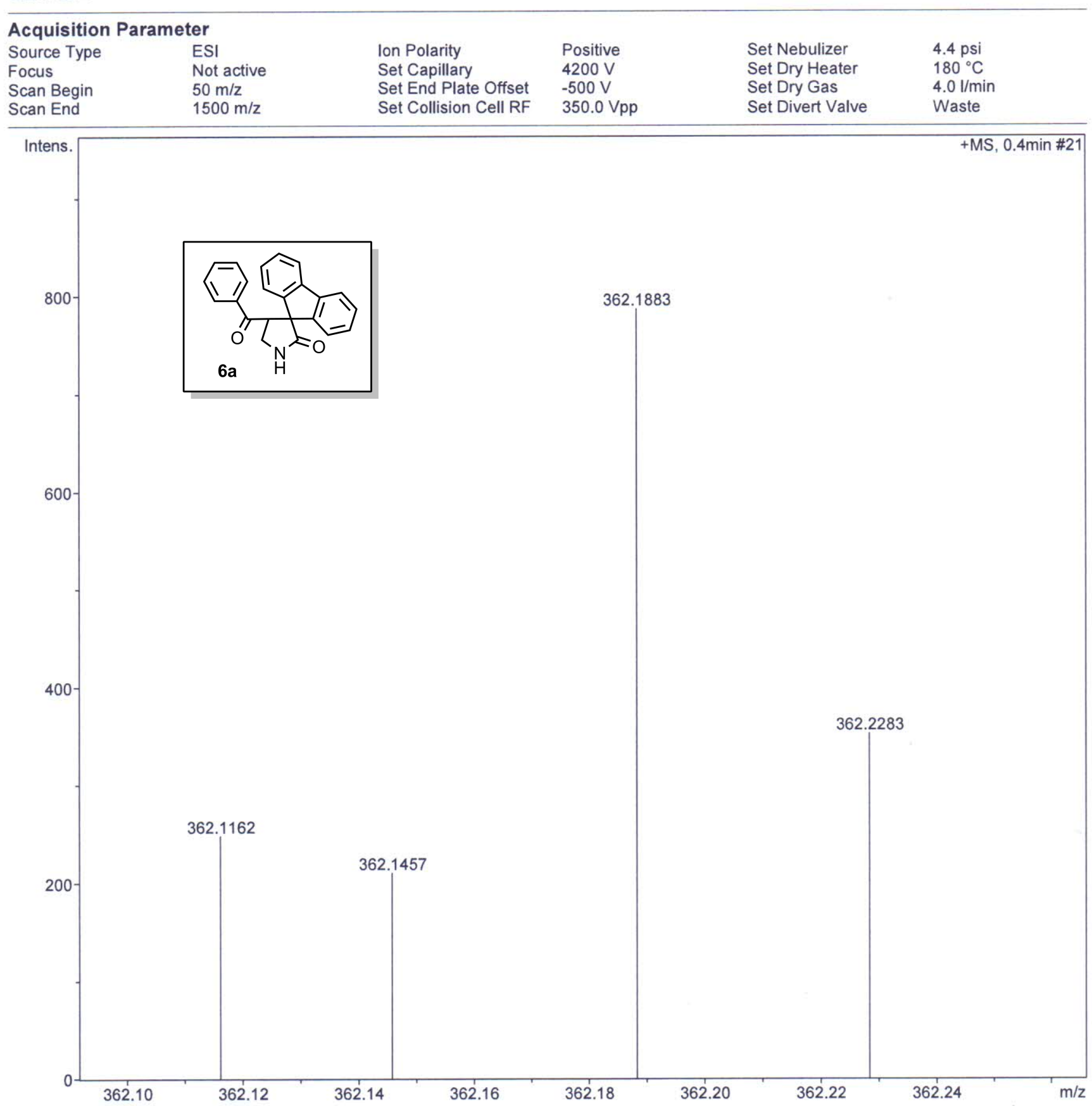




\section{BRUKER MAXIS HRMS REPORT \\ School of Chemistry \\ University of Hyderabad}

Analysis Info

Analysis Name

Method

Sample Name

Comment
D:IDatal2015IDR.AK.SAHOOINOMPB-8-137.d

tune_low_Pos-R2.m

PB-8-137
Acquisition Date

Operator

Instrument
12/1/2015 3:56:17 PM

Ramu Sridhar maXis 10138

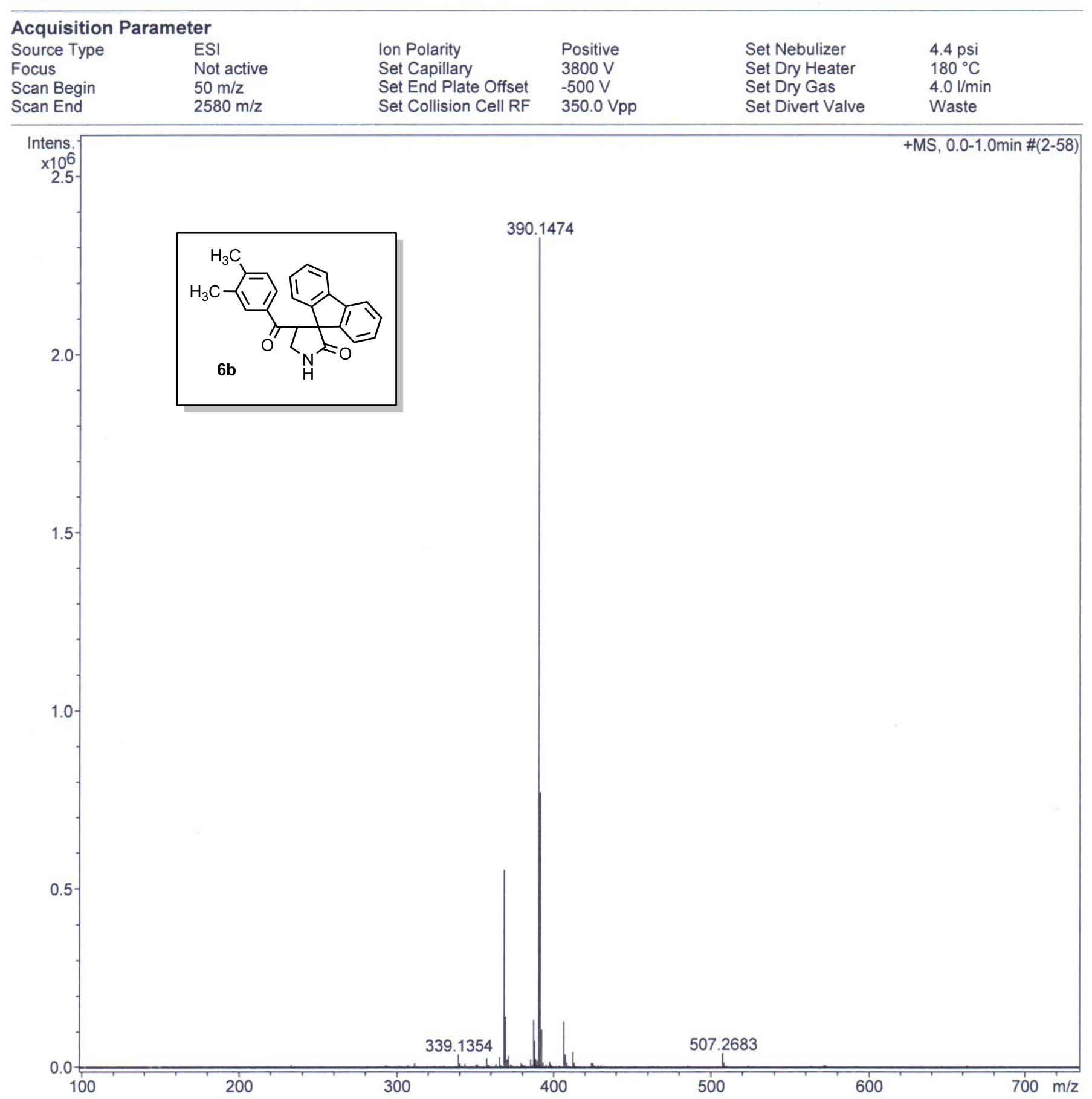




\section{BRUKER MAXIS HRMS REPORT \\ School of Chemistry \\ University of Hyderabad}

\section{Analysis Info}

Analysis Name Method

Sample Name

Comment
Acquisition Date

11/30/2015 4:11:30 PM

Datal2015IDR.AK.SAHOOINOVPB-8-128.d tune_low.m

PB-8-128

Operator

Ramu Sridhar

Instrument

\section{Acquisition Parameter}

Source Type

Focus

Scan Begin

Scan End

ES

Not active

$50 \mathrm{~m} / \mathrm{z}$

$1800 \mathrm{~m} / \mathrm{z}$

$\begin{array}{ll}\text { lon Polarity } & \text { Positive } \\ \text { Set Capillary } & 4500 \mathrm{~V} \\ \text { Set End Plate Offset } & -500 \mathrm{~V} \\ \text { Set Collision Cell RF } & 350.0 \mathrm{Vpp}\end{array}$

Set Nebulize

$4.0 \mathrm{I} / \mathrm{min}$

Waste

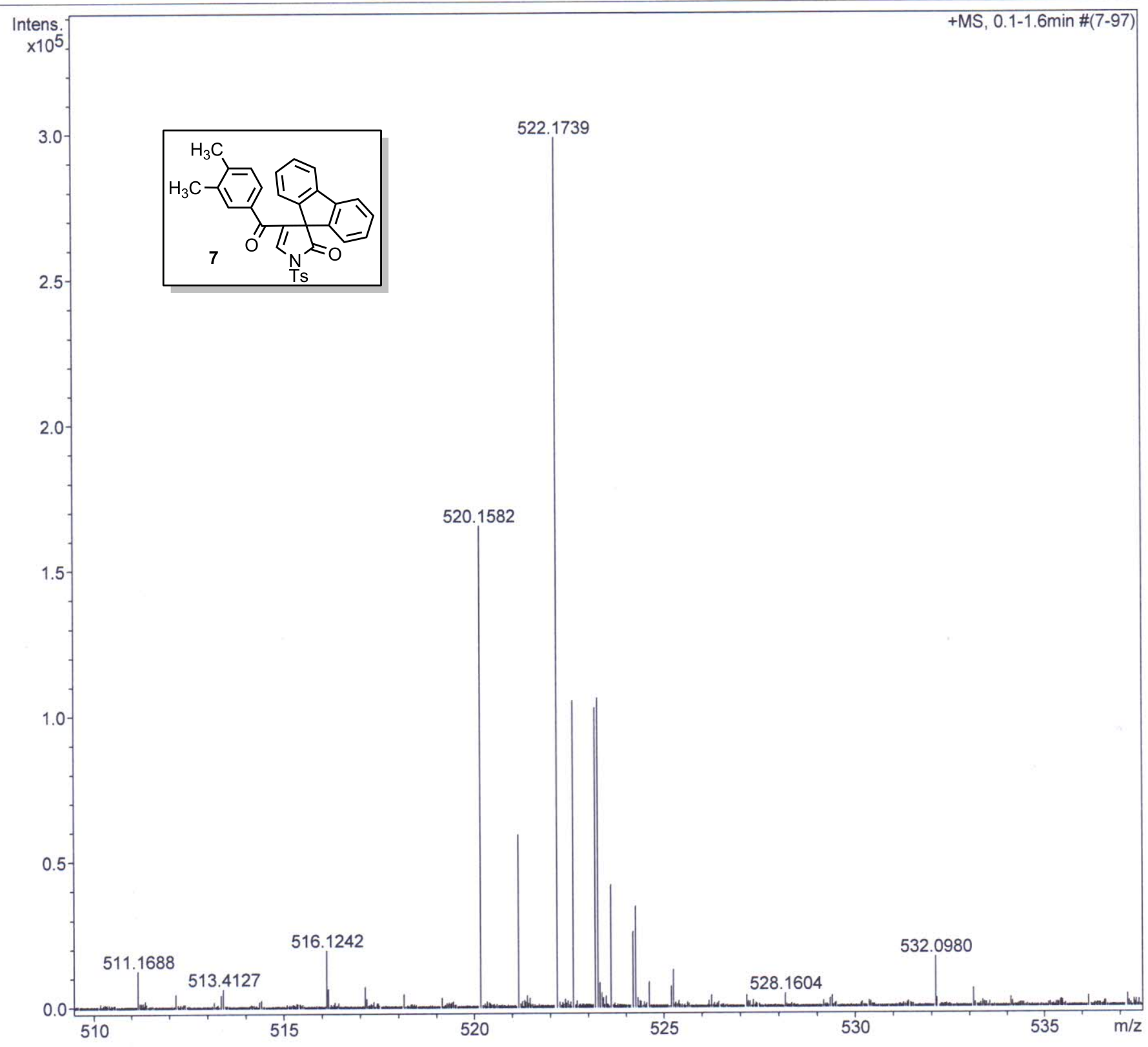


Cyclization of 19 in the presence of $\mathrm{Me}_{2} \mathrm{~S}\left(0^{18}\right.$ ) (Scheme: 7 )

\section{UOH -SCHOOL OF CHEMISTRY -HRMS}

\section{Analysis Info}

Analysis Name Method

Sample Name

Comment
Acquisition Date 6/4/2016 4:25:13 PM

D:IDatal2016IDR.A.K.SAHOOIJUNEIPB-10--84.d tune_low_Pos-R2.m PB-10-84
Operator Rajesh Vashisth

Instrument $\quad$ maXis $\quad 10138$

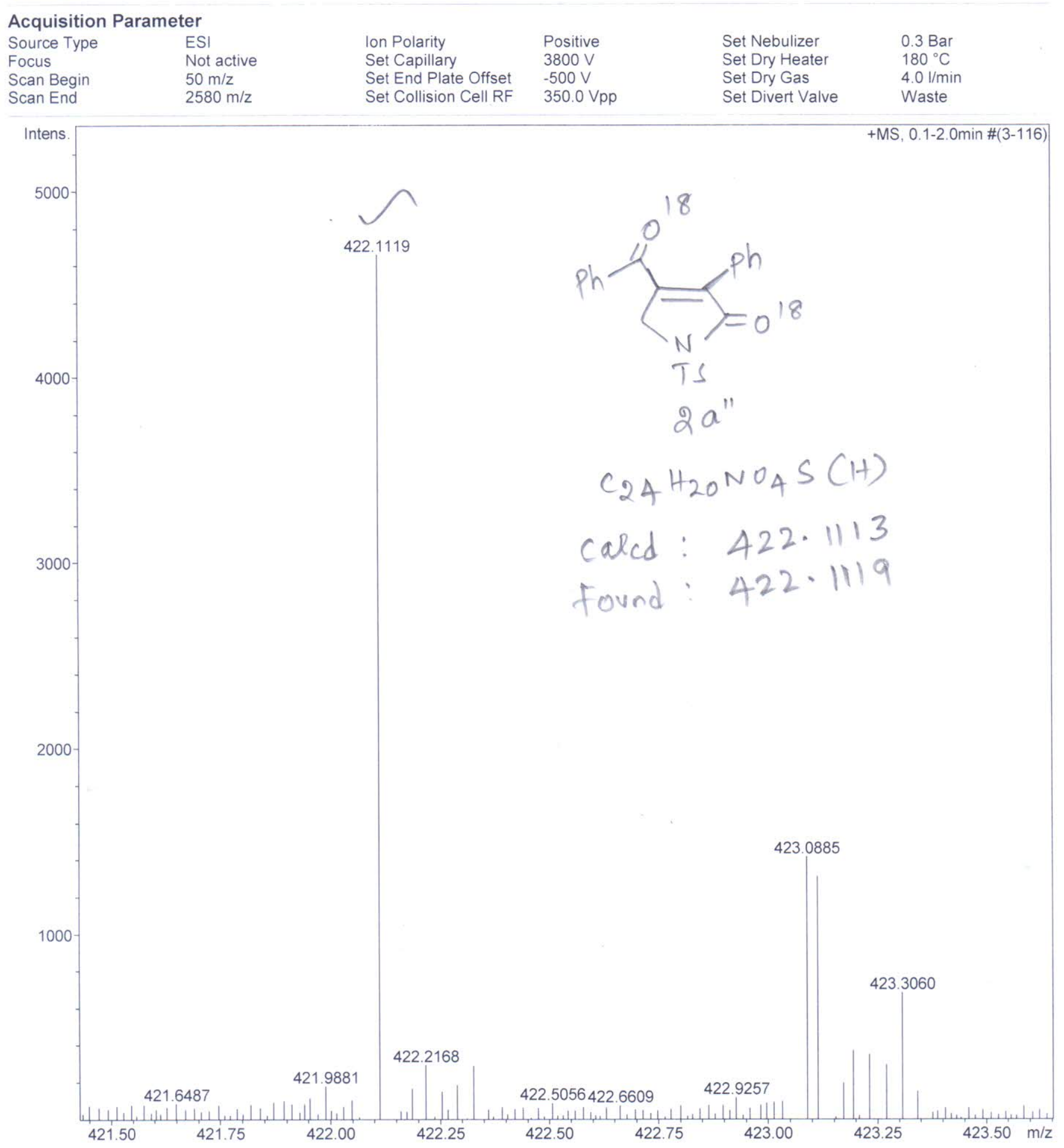

